TRANSACTIONS OF THE

AMERICAN MATHEMATICAL SOCIETY

Volume 362, Number 2, February 2010, Pages 687-726

S 0002-9947(09)04681-9

Article electronically published on September 18, 2009

\title{
VALUE FUNCTIONS AND ASSOCIATED GRADED RINGS FOR SEMISIMPLE ALGEBRAS
}

\author{
J.-P. TIGNOL AND A. R. WADSWORTH
}

\begin{abstract}
We introduce a type of value function $y$ called a gauge on a finitedimensional semisimple algebra $A$ over a field $F$ with valuation $v$. The filtration on $A$ induced by $y$ yields an associated graded ring $\operatorname{gr}_{y}(A)$ which is a graded algebra over the graded field $g_{v}(F)$. Key requirements for $y$ to be a gauge are that $\operatorname{gr}_{y}(A)$ be graded semisimple and that $\operatorname{dim}_{g_{v}(F)}\left(g r_{y}(A)\right)=$ $\operatorname{dim}_{F}(A)$. It is shown that gauges behave well with respect to scalar extensions and tensor products. When $v$ is Henselian and $A$ is central simple over $F$, it is shown that $\operatorname{gr}_{y}(A)$ is simple and graded Brauer equivalent to $\operatorname{gr}_{w}(D)$, where $D$ is the division algebra Brauer equivalent to $A$ and $w$ is the valuation on $D$ extending $v$ on $F$. The utility of having a good notion of value function for central simple algebras, not just division algebras, and with good functorial properties, is demonstrated by giving new and greatly simplified proofs of some difficult earlier results on valued division algebras.
\end{abstract}

\section{INTRODUCTION}

Valuation theory is a time-honored subject, which has undergone a robust development for noncommutative division rings in the last two decades, spurred by its applications to the constructions of noncrossed products and of counterexamples to the Kneser-Tits conjecture; see $\mathrm{W}_{4}$ for a recent and fairly comprehensive survey. However, results that relate valuations with Brauer-group properties have been particularly difficult to establish; a major source of problems is that valuations are defined only on division algebras and not on central simple algebras with zero divisors. The purpose of this work is to introduce a more flexible tool, which we call gauge, inspired by the normes carrées of Bruhat and Tits [BT] (see Rem. 1.21). Gauges are valuation-like maps defined on finite-dimensional semisimple algebras over valued fields with arbitrary value group.

With any valuation there is an associated filtration of the ring, which yields an associated graded ring. Such filtrations and associated graded rings are actually defined not just for valuations, but also for more general value functions: the surmultiplicative value functions defined in (1.4) below, which are sometimes called pseudo-valuations. The gauges we consider here are the surmultiplicative

Received by the editors May 2, 2007 and, in revised form, November 6, 2007.

2000 Mathematics Subject Classification. Primary 16W60; Secondary 16K20, 16W70.

The first author was partially supported by the National Fund for Scientific Research (Belgium) and by the European Community under contract HPRN-CT-2002-00287, KTAGS. The second author would like to thank the first author and UCL for their hospitality while the work for this paper was carried out.

(C)2009 American Mathematical Society Reverts to public domain 28 years from publication 
value functions for which the associated graded algebra is semisimple, and which also satisfy a defectlessness condition; see Def. 1.4 It turns out that gauges exist in abundance and have good behavior with respect to tensor products, but that they still have sufficient uniqueness to reflect the structure of the algebras they are defined on.

Valuation theory typically derives information on fields or division algebras from properties of the residue field or algebra and of the ordered group of values. In a noncommutative setting, these structures interact since the value group acts naturally on the center of the residue algebra; see (1.16). It is therefore reasonable to consider the graded algebra associated with the valuation filtration, which encapsulates information on the residue algebra, the value group, and their interaction. This paper shows how fruitful it can be to work with the graded structures. Associated graded algebras have previously been studied for valuations on division algebras, as in $\mathrm{Bl}_{1}, \mathrm{Bl}_{2}$, and $\mathrm{HW}_{2}$. But they have not been used in the earlier work with value functions on central simple algebras in [BT], nor with the value functions associated to Dubrovin valuation rings in $\mathrm{M}_{2}$. (The relation between the value functions considered here and Morandi's value functions in $\mathrm{M}_{2}$ is described in Prop. 2.5.)

For a given semisimple algebra $A$ over a field $F$, we fix a valuation $v$ on $F$ and consider gauges $y$ on $A$ which restrict to $v$ on $F$, which we call $v$-gauges or (when $v$ is understood) $F$-gauges. The associated graded $\operatorname{ring} \operatorname{gr}_{y}(A)$ is then a finite-dimensional algebra over the graded field $\operatorname{gr}_{v}(F)$. If $A$ is central simple over $F$, there are typically many different $v$-gauges $y$ on $A$; it turns out that $\operatorname{gr}_{y}(A)$ is always a graded simple algebra (i.e., there are no nontrivial homogeneous ideals), and that the class of $g r_{y}(A)$ is uniquely determined in the graded Brauer group of its center; see Cor. 3.7

We get the strongest information when the valuation on $F$ is Henselian. For any finite-dimensional division algebra $D$ over $F$, it is well known that the Henselian valuation $v$ on $F$ has a unique extension to a valuation $w$ on $D$. For $A=\operatorname{End}_{D}(M)$, where $M$ is a finite-dimensional right $D$-vector space, we prove in Theorem 3.1 that for any $v$-gauge $y$ on $A$ there is a norm $\alpha$ (a kind of value function) on $M$ such that up to isomorphism $y$ is the gauge on $\operatorname{End}_{D}(M)$ induced by $\alpha$ on $M$ as described in \$1.3 It follows that $\operatorname{gr}_{y}(A)$ is isomorphic as a graded ring to $\operatorname{End}_{g r_{w}(D)}\left(g r_{\alpha}(M)\right)$; furthermore, the graded Brauer class of $g r_{y}(A)$ is the same as that of $g r_{w}(D)$, and $g_{y}(A)$ has the same matrix size as $A$. In particular, if $A$ is central simple over $F$ and the gauge is tame, in the sense that the center of $\operatorname{gr}_{y}(A)$ is $g r_{v}(F)$, then $g r_{y}(A)$ is a graded central simple algebra over $g_{v}(F)$ with the same Schur index as $A$. We may then consider its Brauer class $\left[\operatorname{gr}_{y}(A)\right]$ in the graded Brauer group $\operatorname{GBr}\left(g r_{v}(F)\right)$. The map $[A] \mapsto\left[g_{y}(A)\right]$ defines an index-preserving group isomorphism $\Psi$ from the tame Brauer group $\operatorname{TBr}(F)$, which is the subgroup of $\operatorname{Br}(F)$ split by the maximal tamely ramified extension of $F$, onto $\operatorname{GBr}\left(\operatorname{gr}_{v}(F)\right)$. That $\Psi$ is an isomorphism was proved earlier in $\left[\mathrm{HW}_{2}\right.$; without the use of gauges the proof in $\left[\mathrm{HW}_{2}\right.$ that $\Psi$ is a group homomorphism was particularly involved and arduous. The proof given here in Theorem 3.8 is much easier and more natural, because we can work with central simple algebras, not just with division algebras, and because gauges work well with tensor products. The map $\Psi$ should be compared with a similar map for Witt groups defined in TW] to generalize Springer's theorem on quadratic forms over complete discretely valued fields. 
When $v$ is Henselian and $A$ is assumed just to be semisimple, we show in Theorem 3.3 that for any $v$-gauge on $A$ the simple components of $g r_{y}(A)$ are the graded algebras for the restrictions of $y$ to the simple components of $A$. Thus, the results described above apply component-by-component. Also, the information obtained in the Henselian case can be extrapolated to gauges with respect to non-Henselian valuations $v$. For, if the valuation $v^{h}$ on field $F^{h}$ is the Henselization of a valuation $v$ on $F$, and $y$ is any $v$-gauge on a semisimple $F$-algebra $A$, then there is a canonical extension of $y$ to a $v^{h}$-gauge $y^{h}$ on $A \otimes_{F} F^{h}$, and $\operatorname{gr}_{y}(A)$ is graded isomorphic to $\operatorname{gr}_{y^{h}}\left(A \otimes_{F} F^{h}\right)$. Thus, any $v$-gauge on $A$ gives insight into what happens with $A$ on passage to the Henselization of $v$.

In the last section, we apply gauges to obtain information on the division algebra Brauer-equivalent to a crossed product or to a tensor product of symbol algebras over valued fields. The idea is that, since we are now freed from the constraint to deal with division algebras, we may easily define gauges on these central simple algebras, and use the associated graded structure to derive properties of their Brauer-equivalent division algebras. We thus easily recover in a straightforward way several results that were previously obtained in $[\mathrm{JW}]$ and $\mathrm{W}_{3}$ by much more complicated arguments.

The organization of the paper is as follows: $\$ 1$ gives the definition of gauges and describes various examples on division algebras, endomorphism algebras, and tensor products. In $₫ 2$ we review some results on graded central simple algebras, complementing the discussion in $\mathrm{HW}_{2}$ with a result characterizing the grade group of the Brauer-equivalent graded division algebra. The main results quoted above, relating semisimple algebras with a gauge over a Henselian field to their associated graded algebras, are given in 3 . This section also contains the definition of the map $\Psi: \operatorname{TBr}(F) \rightarrow \operatorname{GBr}(\operatorname{gr}(F))$. The applications to crossed products and tensor products of symbols are in $\$ 4$

\section{Value functions, NORMs, AND Gauges}

Let $D$ be a division ring finite-dimensional over its center. Let $\Gamma$ be a divisible totally ordered abelian group. Let $\infty$ be an element of a set strictly containing $\Gamma$; extend the ordering on $\Gamma$ to $\Gamma \cup\{\infty\}$ by requiring that $\gamma<\infty$ for each $\gamma \in \Gamma$. Further set $\gamma+\infty=\infty+\infty=\infty$ for all $\gamma \in \Gamma$. A valuation on $D$ is a function $w: D \rightarrow \Gamma \cup\{\infty\}$ satisfying, for all $c, d \in D$,

$$
\begin{gathered}
w(d)=\infty \text { iff } d=0 ; \\
w(c d)=w(c)+w(d) ; \\
w(c+d) \geq \min (w(c), w(d)) .
\end{gathered}
$$

(It follows that $w(1)=w(-1)=0$ and if $w(c) \neq w(d)$, then $w(c+d)=$ $w(c-d)=\min (w(c), w(d))$.) Associated to the valuation on $D$, we have its value group $\Gamma_{D}=w\left(D^{\times}\right)$, where $D^{\times}$is the group of units of $D$, i.e., $D^{\times}=D-\{0\}$; its valuation ring $V_{D}=\{d \in D \mid w(d) \geq 0\}$; the unique maximal left (and right) ideal $M_{D}$ of $V_{D}, M_{D}=\{d \in D \mid w(d)>0\}$; and the residue division ring $\bar{D}=V_{D} / M_{D}$. Another key structure is the associated graded ring: for $\gamma \in \Gamma$, set $D^{\geq \gamma}=\{d \in D \mid w(d) \geq \gamma\}$ and $D^{>\gamma}=\{d \in D \mid w(d)>\gamma\}$, which is a subgroup of $D^{\geq \gamma}$; let $D_{\gamma}=D^{\geq \gamma} / D^{>\gamma}$. The associated graded ring of $D$ with respect to $w$ is $g r_{w}(D)=\bigoplus_{\gamma \in \Gamma} D_{\gamma}$. For each $\gamma, \delta \in \Gamma$, the multiplication in $D$ induces a well-defined 
multiplication $D_{\gamma} \times D_{\delta} \rightarrow D_{\gamma+\delta}$ given by $\left(c+D^{>\gamma}\right) \cdot\left(d+D^{>\delta}\right)=c d+D^{>\gamma+\delta}$. This multiplication is extended biadditively to all of $g r_{w}(D)$, making $g r_{w}(D)$ into a graded ring. When $w$ is clear, we write $\operatorname{gr}(D)$ for $g r_{w}(D)$. The grade group of $\operatorname{gr}(D)$, denoted $\Gamma_{\operatorname{gr}(D)}$, is $\left\{\gamma \in \Gamma \mid D_{\gamma} \neq 0\right\}$; note that $\Gamma_{\operatorname{gr}(D)}=\Gamma_{D}$. Also, for the degree 0 component of $\operatorname{gr}(D)$, we have $D_{0}=D^{\geq 0} / D^{>0}=V_{D} / M_{D}=\bar{D}$. For $d \in D^{\times}$, we write $d^{\prime}$ for the image of $d$ in $\operatorname{gr}(D)$, i.e., $d^{\prime}=d+D^{>w(d)} \in D_{w(d)}$. The homogeneous elements of $\operatorname{gr}(D)$ are those in $\bigcup_{\gamma \in \Gamma} D_{\gamma}$. It follows from property (1.1) that $\operatorname{gr}(D)$ is a graded division ring, i.e., every nonzero homogeneous element of $\operatorname{gr}(D)$ is a unit.

Now, let $M$ be a right $D$-vector space, where $D$ has a valuation $w$. A function $\alpha: M \rightarrow \Gamma \cup\{\infty\}$ is called a D-value function with respect to $w$ (or a $w$-value function) if for all $m, n \in M$ and $d \in D$,

$$
\begin{aligned}
\alpha(m) & =\infty \text { iff } m=0 ; \\
\alpha(m d) & =\alpha(m)+w(d) ; \\
\alpha(m+n) & \geq \min (\alpha(m), \alpha(n)) .
\end{aligned}
$$

Given such an $\alpha$ on $M$, we can form the associated graded module $\operatorname{gr}_{\alpha}(M)$ just as before: for $\gamma \in \Gamma$, let $M^{\geq \gamma}=\{m \in M \mid \alpha(m) \geq \gamma\}$ and $M^{>\gamma}=\{m \in$ $M \mid \alpha(m)>\gamma\}$; then set $M_{\gamma}=M^{\geq \gamma} / M^{>\gamma}$. Define $\operatorname{gr}(M)=g r_{\alpha}(M)=\bigoplus_{\gamma \in \Gamma} M_{\gamma}$.

For nonzero $m \in M$, let $m^{\prime}$ denote the image $m+M^{>\alpha(m)}$ of $m$ in $\operatorname{gr}(M)$; for $0 \in M$, let $0^{\prime}=0 \in \operatorname{gr}(M)$. For $\gamma, \delta \in \Gamma$ there is a well-defined multiplication $M_{\gamma} \times D_{\delta} \rightarrow M_{\gamma+\delta}$ given by $\left(m+M^{>\gamma}\right) \cdot\left(d+D^{>\delta}\right)=(m d)+M^{>\gamma+\delta}$. This is extended distributively to yield an operation $\operatorname{gr}(M) \times \operatorname{gr}(D) \rightarrow \operatorname{gr}(M)$ which makes $\operatorname{gr}(M)$ into a graded right $\operatorname{gr}(D)$-module. It is well known and easy to prove by a slight variation of the ungraded argument that every graded module over a graded division ring is a free module with a homogeneous base, and every two bases have the same cardinality. Thus, graded modules over graded division rings are called graded vector spaces; we write $\operatorname{dim}_{\operatorname{gr}(D)}(\operatorname{gr}(M))$ for the cardinality of any $\operatorname{gr}(D)$ module base of $\operatorname{gr}(M)$. If $N=\bigoplus_{\gamma \in \Gamma} N_{\gamma}$ is another graded right $\operatorname{gr}(D)$-vector space, we say that $M$ and $N$ are graded isomorphic, written $M \cong_{g} N$, if there is a $\operatorname{gr}(D)$ vector space isomorphism $f: M \rightarrow N$ with $f\left(M_{\gamma}\right)=N_{\gamma}$ for each $\gamma \in \Gamma$.

Now, suppose $M$ is finite-dimensional. A right $D$-vector space base $\left(m_{i}\right)_{1 \leq i \leq k}$ of $M$ is called a splitting base with respect to $\alpha$ if for all $d_{1}, \ldots, d_{k} \in D$,

$$
\alpha\left(\sum_{i=1}^{k} m_{i} d_{i}\right)=\min _{1 \leq i \leq k}\left(\alpha\left(m_{i}\right)+w\left(d_{i}\right)\right) .
$$

If there is a splitting base for the $D$-value function $\alpha$, we say that $\alpha$ is a $D$-norm (or a $w$-norm) on $M$. Note that it is easy to construct $D$-norms on $M$ : take any $D$ vector space base $\left(m_{i}\right)_{1 \leq i \leq k}$ of $M$, and take any $\gamma_{1}, \ldots, \gamma_{k} \in \Gamma$. Define $\alpha\left(m_{i}\right)=\gamma_{i}$ for $1 \leq i \leq k$, and then define $\alpha$ on all of $M$ by formula (1.3). It is straightforward to check that such an $\alpha$ is a $D$-norm on $M$ and $\left(m_{i}\right)_{1 \leq i \leq k}$ is a splitting base for $\alpha$. 
Recall the following from [RTW, Prop. 2.2, Cor. 2.3, Prop. 2.5]:

Proposition 1.1. Let $\alpha$ be a $D$-value function on $M$. Take any $m_{1}, \ldots, m_{\ell} \in M$.

(i) $m_{1}^{\prime}, \ldots, m_{\ell}^{\prime}$ are $\operatorname{gr}(D)$-linearly independent in $\operatorname{gr}(M)$ iff $\alpha\left(\sum_{i=1}^{\ell} m_{i} d_{i}\right)=$ $\min _{1 \leq i \leq \ell}\left(\alpha\left(m_{i}\right)+w\left(d_{i}\right)\right)$ for all $d_{1}, \ldots, d_{\ell} \in D$. When this occurs, $m_{1}, \ldots$, $m_{\ell}$ are $D$-linearly independent in $M$.

(ii) $\operatorname{dim}_{\operatorname{gr}(D)}(\operatorname{gr}(M)) \leq \operatorname{dim}_{D}(M)$. Equality holds iff $\alpha$ is a $D$-norm on $M$.

(iii) Suppose $\alpha$ is a D-norm on $M$. Then, for any $D$-subspace $N$ of $M,\left.\alpha\right|_{N}$ is a norm on $N$.

We are interested here in value functions on algebras. Let $F$ be a field with valuation $v: F \rightarrow \Gamma \cup\{\infty\}$, and let $A$ be a finite-dimensional $F$-algebra. A function $y: A \rightarrow \Gamma \cup\{\infty\}$ is called a surmultiplicative $F$-value function on $A$ if for any $a$, $b \in A$,

$$
\begin{gathered}
y(1)=0, \text { and } y(a)=\infty \text { iff } a=0 ; \\
y(c a)=v(c)+y(a) \text { for any } c \in F ; \\
y(a+b) \geq \min (y(a), y(b)) \\
y(a b) \geq y(a)+y(b) .
\end{gathered}
$$

Note that for such a $y$, there is a corresponding "valuation ring" $V_{A}=A^{\geq 0}=\{a \in$ $A \mid y(a) \geq 0\}$. There is also an associated graded ring $\operatorname{gr}(A)=\operatorname{gr}_{y}(A)=\bigoplus_{\gamma \in \Gamma} A_{\gamma}$, where $A_{\gamma}=A^{\geq \gamma} / A^{>\gamma}$, as above, and the multiplication in $\operatorname{gr}(A)$ is induced by that of $A$. Furthermore, $\operatorname{gr}_{y}(A)$ is clearly a graded $g r_{v}(F)$-algebra. Also, $g r_{v}(F)$ is a graded field, i.e., a commutative graded ring in which every nonzero homogeneous element is a unit. Since axioms $(1.4 \mathrm{a})-(1.4 \mathrm{c})$ show that $y$ is an $F$-value function for $A$ as an $F$-vector space, Prop. 1.1(ii) implies that $\operatorname{dim}_{\operatorname{gr}(F)}(\operatorname{gr}(A)) \leq \operatorname{dim}_{F}(A)$, with equality iff $y$ is an $F$-norm on $A$. The following lemma is convenient for verifying when an $F$-norm on $A$ is surmultiplicative:

Lemma 1.2. Suppose $y: A \rightarrow \Gamma \cup\{\infty\}$ is an F-norm on $A$ such that $y(1)=0$. Let $\left(a_{i}\right)_{1 \leq i \leq k}$ be a splitting base of $A$. If $y\left(a_{i} a_{j}\right) \geq y\left(a_{i}\right)+y\left(a_{j}\right)$ for all $i, j$, then $y$ is a surmultiplicative $F$-value function on $A$.

Proof. We need only to verify axiom (1.4d). For this, take any $b_{1}=\sum c_{i} a_{i}$ and $b_{2}=\sum d_{i} a_{i}$ in $A$ with $c_{i}, d_{i} \in F$. Then,

$$
\begin{aligned}
y\left(b_{1} b_{2}\right) & =y\left(\sum_{i, j} c_{i} d_{j} a_{i} a_{j}\right) \geq \min _{i, j}\left(y\left(c_{i} d_{j} a_{i} a_{j}\right)\right) \\
& \geq \min _{i, j}\left(v\left(c_{i}\right)+v\left(d_{j}\right)+y\left(a_{i}\right)+y\left(a_{j}\right)\right) \\
& \geq \min _{i}\left(v\left(c_{i}\right)+y\left(a_{i}\right)\right)+\min _{j}\left(v\left(d_{j}\right)+y\left(a_{j}\right)\right)=y\left(b_{1}\right)+y\left(b_{2}\right) .
\end{aligned}
$$

If $A$ has a surmultiplicative value function $y$, then for nonzero $a \in A$, we write $a^{\prime}$ for the image $a+A^{>y(a)}$ of $a$ in $A_{y(a)}$. For 0 in $A$, we write $0^{\prime}=0 \in \operatorname{gr}(A)$. The following immediate consequence of the definitions will be used repeatedly below: for nonzero $a, b \in A$ :

$$
a^{\prime} b^{\prime}= \begin{cases}(a b)^{\prime}, & \text { if } a^{\prime} b^{\prime} \neq 0^{\prime}, \text { iff } y(a b)=y(a)+y(b) \\ 0^{\prime}, & \text { if } y(a b)>y(a)+y(b) .\end{cases}
$$


With this, we can readily characterize the inverse image in $A$ of the group of homogeneous units of $\operatorname{gr}(A)$ :

Lemma 1.3. Let y be a surmultiplicative $F$-value function on a finite-dimensional $F$-algebra $A$. For any nonzero $u \in A$, the following conditions are equivalent:

(i) $u^{\prime} \in \operatorname{gr}(A)^{\times}$, the group of units of $\operatorname{gr}(A)$;

(ii) $y(a u)=y(a)+y(u)$ for all $a \in A$;

(ii') $y(u a)=y(u)+y(a)$ for all $a \in A$;

(iii) $u \in A^{\times}$and $y(u)+y\left(u^{-1}\right)=0$.

Proof. (i) $\Rightarrow$ (ii) Suppose $u^{\prime} \in \operatorname{gr}(A)^{\times}$. Then, for any nonzero $a \in A$, we have $a^{\prime} u^{\prime} \neq 0^{\prime}$; hence, $y(a u)=y(a)+y(u)$ by (1.5). (ii) $\Rightarrow$ (i) By (1.5), (ii) implies that $a^{\prime} u^{\prime} \neq 0^{\prime}$ for every nonzero $a \in A$. Therefore, as $\operatorname{gr}(A)$ is a finite-dimensional graded algebra over the graded field $\operatorname{gr}(F), u^{\prime} \in \operatorname{gr}(A)^{\times}$. (i) $\Leftrightarrow\left(\mathrm{ii}^{\prime}\right)$ is proved analogously. (ii) $\Rightarrow$ (iii) Condition (ii) shows that $u$ is not a zero divisor in the finite-dimensional algebra $A$. Therefore, $u \in A^{\times}$. The formula in (iii) follows by setting $a=u^{-1}$ in (ii). (iii) $\Rightarrow$ (ii) For any $a \in A$, we have $y(a)=y\left(a u u^{-1}\right) \geq y(a u)+y\left(u^{-1}\right)$. Therefore, (iii) yields

$$
y(a u) \leq y(a)-y\left(u^{-1}\right)=y(a)+y(u) \leq y(a u) ;
$$

so equality holds throughout, proving (ii).

It is easy to construct numerous surmultiplicative value functions $y$ on $A$ using Lemma 1.2. We next make further restrictions on $y$ so as to be able to relate the structure of $\operatorname{gr}(A)$ to that of $A$.

If $K$ is a graded field, then a finite-dimensional graded $K$-algebra $B$ is said to be graded simple if $B$ has no homogeneous two-sided ideals except $B$ and $\{0\}$. We say that $B$ is a graded semisimple $K$-algebra if $B$ is a direct product of finitely many graded simple $K$-algebras. By a variation of the ungraded argument, this is equivalent to: $B$ has no nonzero nilpotent homogeneous ideals.

If $B$ is an algebra (resp. graded algebra) over a field (resp. graded field) $K$, we write $[B: K]$ for $\operatorname{dim}_{K}(B)$. Throughout the paper, all semisimple (resp. graded semisimple) algebras are tacitly assumed to be finite-dimensional.

Definition 1.4. Let $F$ be a field with a valuation $v$. Let $y$ be a surmultiplicative value function on a finite-dimensional $F$-algebra $A$. We say that $y$ is an $F$-gauge (or a $v$-gauge) on $A$ if $y$ is an $F$-norm on $A$ (i.e., $[\operatorname{gr}(A): \operatorname{gr}(F)]=[A: F])$ and $\operatorname{gr}(A)$ is a graded semisimple $\operatorname{gr}(F)$-algebra. Note that if $A$ has an $F$-gauge, then $A$ must be semisimple. For, if $A$ had a nonzero ideal $N$ with $N^{2}=\{0\}$, then $\operatorname{gr}(N)$ would be a nonzero ideal of $\operatorname{gr}(A)$ with $\operatorname{gr}(N)^{2}=\{0\}$.

For any ring $R$, let $Z(R)$ denote the center of $R$.

Definition 1.5. An $F$-gauge $y$ on a finite-dimensional semisimple $F$-algebra $A$ is called a tame $F$-gauge if $Z(\operatorname{gr}(A))=\operatorname{gr}(Z(A))$ and $Z(\operatorname{gr}(A))$ is separable over $\operatorname{gr}(F)$. Just as in the ungraded case, $Z(\operatorname{gr}(A)) \cong \cong_{g} K_{1} \times \ldots \times K_{n}$, where each $K_{i}$ is a graded field which is the center of a simple component of $A$. Also, $Z(\operatorname{gr}(A))$ is separable over $\operatorname{gr}(F)$ if and only if each $K_{i}$ is separable over $F$. By $\mathrm{HW}_{1}$, Th. 3.11], this holds if and only if $K_{i, 0}$ is separable over $\operatorname{gr}(F)_{0}$ and $\operatorname{char}\left(\operatorname{gr}(F)_{0}\right) \nmid\left|\Gamma_{K_{i}}: \Gamma_{F}\right|$. Thus, the gauge is tame if and only if $Z(\operatorname{gr}(A))=\operatorname{gr}(Z(A)), \overline{Z(A)}$ is separable over $\bar{F}$, and for each simple component $K_{i}$ of $\operatorname{gr}(Z(A)), \operatorname{char}(\bar{F}) \nmid\left|\Gamma_{K_{i}}: \Gamma_{F}\right|$. It will be shown below (see Cor. 3.6) that whenever $\operatorname{char}(\bar{F})=0$ every $F$-gauge is tame. 
While gauges are defined for semisimple algebras, the next proposition shows that the study of gauges can be reduced to the case of simple algebras.

Proposition 1.6. Let $F$ be a field with valuation $v$, and let $A$ be a semisimple $F$-algebra with an F-gauge $y$. Suppose $A$ is a direct product of F-subalgebras

$$
A=B_{1} \times \ldots \times B_{k} .
$$

Then, $\left.y\right|_{B_{i}}$ is a gauge for each $i, y\left(b_{1}, \ldots, b_{n}\right)=\min \left(y\left(b_{1}\right), \ldots, y\left(b_{n}\right)\right)$, and

$$
\operatorname{gr}(A) \cong \operatorname{gr}\left(B_{1}\right) \times \ldots \times \operatorname{gr}\left(B_{n}\right) .
$$

Furthermore, $y$ is tame if and only if each $\left.y\right|_{B_{i}}$ is tame.

The proof of Prop. [1.6 will invoke the following easy but very useful lemma:

Lemma 1.7. Let $F$ be a field with a valuation $v$, and let $A$ be an $F$-algebra with a surmultiplicative $F$-value function $y$. Suppose there is $e \in A$ with $e^{2}=e$ and $y(e)=0$. Then for any $F$-subspace $N$ of $A$,

$$
\operatorname{gr}(e N)=e^{\prime} \operatorname{gr}(N) \quad \text { and } \quad \operatorname{gr}(N e)=\operatorname{gr}(N) e^{\prime} .
$$

Proof. For any $s \in N$, we have $e^{\prime} s^{\prime}=(e s)^{\prime}$ or $e^{\prime} s^{\prime}=0$, by (1.5). Hence, $e^{\prime} \operatorname{gr}(N) \subseteq$ $\operatorname{gr}(e N)$. On the other hand, $y(e(e s))=y(e s)=y(e)+y(e s)$. Hence, $e^{\prime}(e s)^{\prime}=$ $(e(e s))^{\prime}=(e s)^{\prime}$, showing that $\operatorname{gr}(e N)=e^{\prime} \operatorname{gr}(e N) \subseteq e^{\prime} \operatorname{gr}(N)$. This proves the first equality in (1.6), and the second is proved analogously.

Proof of Prop. 1.6. It suffices by induction to prove the case $n=2$. Therefore, assume $A=B \times C$ with $B$ and $C$ nontrivial subalgebras of $A$. Let $e=\left(1_{B}, 0\right)$ and $f=\left(0,1_{C}\right)$ in $A$. So, $e$ and $f$ are nonzero orthogonal central idempotents of $A$ with $e+f=1$, and $B=A e=e A, C=f A=A f$.

Consider $e^{\prime} \in \operatorname{gr}(A)$. Since $e \in Z(A)$, we have $e^{\prime} \in Z(\operatorname{gr}(A))$. Also, $y(e) \leq 0$, as $y(e)=y\left(e^{2}\right) \geq y(e)+y(e)$. If $y(e)<0$, then $y\left(e^{2}\right)>y(e)+y(e)$, so $e^{\prime 2}=0$. But then, as $e^{\prime}$ is central, $e^{\prime} \operatorname{gr}(A)$ is a nonzero homogeneous nilpotent ideal of $\operatorname{gr}(A)$, contradicting the semisimplicity of $\operatorname{gr}(A)$. Therefore, we must have $y(e)=$ 0 ; hence, as $y\left(e^{2}\right)=0=y(e)+y(e)$, we have $e^{\prime 2}=\left(e^{2}\right)^{\prime}=e^{\prime}$. Thus, $e^{\prime}$ is a nonzero homogeneous central idempotent of $\operatorname{gr}(A)$. Likewise, the same is true for $f^{\prime}$. Furthermore, as $y(e)=y(f)=y(e+f)=0$ we have $e^{\prime}+f^{\prime}=1^{\prime}$ in $\operatorname{gr}(F)$. Therefore,

$$
\operatorname{gr}(A) \cong g e^{\prime} \operatorname{gr}(A) \times f^{\prime} \operatorname{gr}(A) .
$$

By Lemma 1.7, $e^{\prime} \operatorname{gr}(A)=\operatorname{gr}(e A)=\operatorname{gr}(B)$; likewise, $f^{\prime} \operatorname{gr}(A)=\operatorname{gr}(C)$. Thus, (1.7) becomes

$$
\operatorname{gr}(A) \cong_{g} \operatorname{gr}(B) \times \operatorname{gr}(C) .
$$

Since $y$ is a norm on $A,\left.y\right|_{B}$ and $\left.y\right|_{C}$ are norms by Prop. 1.1(iii). From the direct decomposition of $\operatorname{gr}(A)$, Prop. 1.1 also shows that $B$ and $C$ are splitting complements in $A$ for $y$, i.e., $y(b, c)=\min (y(b), y(c))$ for all $b \in B, c \in C$. Furthermore, $\operatorname{gr}(B)$ and $\operatorname{gr}(C)$ must be graded semisimple, since they are direct factors of $\operatorname{gr}(A)$, which is graded semisimple. Thus, $\left.y\right|_{B}$ and $\left.y\right|_{C}$ are $F$-gauges. We have $Z(A)=Z(B) \times Z(C)$ and $\left.Z(\operatorname{gr}(A)) \cong_{g} Z(\operatorname{gr}(B)) \times \operatorname{gr}(C)\right)=Z(\operatorname{gr}(B)) \times Z(\operatorname{gr}(C))$, so $Z(\operatorname{gr}(A))$ is separable over $\operatorname{gr}(F)$ if and only if $Z(\operatorname{gr}(B))$ and $Z(\operatorname{gr}(C))$ are each separable over $\operatorname{gr}(F)$. Thus, by the definition, $y$ is tame if and only if $\left.y\right|_{B}$ and $\left.y\right|_{C}$ are each tame. 
The notion of gauge generalizes that of defectless valuation on division algebras, and tame gauge generalizes tame valuation. We make this point clear in $\$ 1.2$ and give fundamental examples of gauges on endomorphism algebras and on tensor products in $\S \S 1.3$ and 1.4 . We start our discussion of examples with commutative semisimple algebras.

1.1. Gauges on commutative algebras. For a commutative finite-dimensional algebra $A$ over a field $F$, semisimplicity is equivalent to the absence of nonzero elements $x \in A$ such that $x^{2}=0$. A similar observation holds for graded algebras. Thus, if $F$ has a valuation $v$ and $A$ has a surmultiplicative $v$-value function $y$, the following conditions are equivalent:

(a) $\operatorname{gr}_{y}(A)$ is semisimple;

(b) $\left(x^{\prime}\right)^{2} \neq 0$ for all nonzero $x \in A$;

(c) $\left(x^{\prime}\right)^{n} \neq 0$ for all nonzero $x \in A$ and for every positive integer $n$.

In view of (1.5), these conditions are also equivalent to:

(d) $y\left(x^{2}\right)=2 y(x)$ for all $x \in A$;

(e) $y\left(x^{n}\right)=n y(x)$ for all $x \in A$.

We first consider the case where $A$ is a field.

Proposition 1.8. Let $(F, v)$ be a valued field and let $K / F$ be a finite-degree field extension. Suppose $y: K \rightarrow \Gamma \cup\{\infty\}$ is a surmultiplicative $v$-value function such that $\operatorname{gr}_{y}(K)$ is semisimple. Then, there exist valuations $v_{1}, \ldots, v_{n}$ on $K$ extending $v$ such that

$$
y(x)=\min _{1 \leq i \leq n}\left(v_{i}(x)\right) \quad \text { for } x \in K .
$$

Moreover, there is a natural graded isomorphism of graded gr $(F)$-algebras

$$
g r_{y}(K) \cong g r_{v_{1}}(K) \times \ldots \times g r_{v_{n}}(K) .
$$

Proof. Let $\Gamma_{K}=y\left(K^{\times}\right) \subseteq \Gamma$ and $\Gamma_{F}=v\left(F^{\times}\right)=y\left(F^{\times}\right) \subseteq \Gamma_{K}$. If $x_{1}, \ldots, x_{r} \in K$ are such that $y\left(x_{1}\right), \ldots, y\left(x_{r}\right)$ belong to different cosets of $\Gamma_{F}$ in $\Gamma_{K}$, then $x_{1}^{\prime}$, $\ldots, x_{r}^{\prime} \in \operatorname{gr}(K)$ are linearly independent over $\operatorname{gr}(F)$; hence $x_{1}, \ldots, x_{r}$ are linearly independent over $F$ (see Prop. $1.1(\mathrm{i}))$. Since $[K: F]$ is finite, it follows that the index $\left|\Gamma_{K}: \Gamma_{F}\right|$ is finite; hence $\Gamma_{K} / \Gamma_{F}$ is torsion.

Let $V_{F}$ and $M_{F}$ denote the valuation ring of $F$ and its maximal ideal, and let

$$
V_{y}=\{x \in K \mid y(x) \geq 0\} \quad \text { and } \quad M_{y}=\{x \in K \mid y(x)>0\} .
$$

Clearly, $V_{y}$ is a subring of $K$ containing $V_{F}$ and $M_{y}$ is an ideal of $V_{y}$ containing $M_{F}$. Since $g_{y}(K)$ is assumed to be semisimple, we have $y\left(x^{n}\right)=n y(x)$ for all $x \in K$ (see condition (e) above); hence the ideal $M_{y}$ is radical. We may therefore find a set of prime ideals $P_{\lambda} \subseteq V_{y}$ (indexed by some set $\Lambda$ ) such that $M_{y}=\bigcap_{\lambda \in \Lambda} P_{\lambda}$.

By Chevalley's Extension Theorem [EP Th. 3.1.1], we may find for each $\lambda \in \Lambda$ a valuation ring $V_{\lambda}$ of $K$ with maximal ideal $M_{\lambda}$ such that $V_{y} \subseteq V_{\lambda}$ and $P_{\lambda}=V_{y} \cap M_{\lambda}$.

Claim: The valuation $v_{\lambda}$ corresponding to $V_{\lambda}$ extends $v$. We have $V_{F} \subseteq V_{y} \subseteq V_{\lambda}$; hence $V_{F} \subseteq V_{\lambda} \cap F$. Similarly, $M_{F} \subseteq M_{y} \subseteq P_{\lambda} \subseteq M_{\lambda}$, so $M_{F} \subseteq M_{\lambda} \cap F$. Since $V_{\lambda} \cap F$ is a valuation ring of $F$ with maximal ideal $M_{\lambda} \cap F$, the inclusions $V_{F} \subseteq V_{\lambda} \cap F$ and $M_{F} \subseteq M_{\lambda} \cap F$ imply $V_{F}=V_{\lambda} \cap F$, proving the claim. Hence, each value group $\Gamma_{K, v_{i}}$ embeds canonically into the divisible group $\Gamma$. 
Since there are only finitely many extensions of $v$ to $K$, and since for $\lambda, \lambda^{\prime} \in \Lambda$ the equality $V_{\lambda}=V_{\lambda^{\prime}}$ implies $M_{\lambda}=M_{\lambda^{\prime}}$, hence $P_{\lambda}=P_{\lambda^{\prime}}$, it follows that $\Lambda$ is a finite set. Let $\Lambda=\{1, \ldots, n\}$ and, for $x \in K$, let

$$
w(x)=\min _{1 \leq i \leq n}\left(v_{i}(x)\right) .
$$

Since $V_{y} \subseteq \bigcap_{i=1}^{n} V_{i}$, we have

$$
y(x) \geq 0 \Rightarrow w(x) \geq 0 \quad \text { for } x \in K .
$$

Similarly, since $M_{y}=\bigcap_{i=1}^{n} P_{i}=V_{y} \cap\left(\bigcap_{i=1}^{n} M_{i}\right)$, we have

$$
y(x)>0 \Longleftrightarrow(y(x) \geq 0 \text { and } w(x)>0) .
$$

It follows that

$$
y(x)=0 \Rightarrow w(x)=0 \quad \text { for } x \in K^{\times} .
$$

Now, fix some $x \in K^{\times}$. Since $\Gamma_{K} / \Gamma_{F}$ is a torsion group we may find an integer $m>0$ and an element $u \in F^{\times}$such that $m y(x)=v(u)$; hence $y\left(x^{m} u^{-1}\right)=$ 0 . By (1.9), we then have $w\left(x^{m} u^{-1}\right)=0$. Because each $v_{i}$ extends $v$, we have $w\left(x^{m} u^{-1}\right)=w\left(x^{m}\right)-v(u)$. Hence,

$$
m w(x)=w\left(x^{m}\right)=v(u)=m y(x) .
$$

Since $\Gamma$ has no torsion, it follows that $w(x)=y(x)$, which proves (1.8).

For $i=1, \ldots, n$ we have $y(x) \leq v_{i}(x)$ for all $x \in K$; hence the identity map on $K$ induces a map $g r_{y}(K) \rightarrow g r_{v_{i}}(K)$. Combining these maps, we obtain a graded homomorphism of graded $\operatorname{gr}(F)$-algebras

$$
g r_{y}(K) \rightarrow g r_{v_{1}}(K) \times \ldots \times g r_{v_{n}}(K) .
$$

This map is injective since for every $x \in K^{\times}$there is some index $i$ such that $y(x)=v_{i}(x)$. For surjectivity, fix any $k, 1 \leq k \leq n$ and any $b \in K^{\times}$.

Claim: There is $c \in K^{\times}$with $v_{k}(c)=v_{k}(b)$ and $v_{i}(c) \geq v_{k}(b)$ for $i \neq k$. This will be proved below.

Now, since the valuations $v_{1}, \ldots, v_{n}$ are incomparable and $V_{y}=\bigcap_{i=1}^{n} V_{i}$ by (1.8), the map $V_{y} \rightarrow \prod_{i=1}^{n} V_{i} / M_{i}$ is surjective by [EP, Th. 3.2.7(3)]. Therefore, there is $d \in V_{y}$ with $d \in M_{i}$ for $i \neq k$ and $d-b c^{-1} \in M_{k}$. Let $a=c d$. Then, $v_{i}(a)>v_{i}(c) \geq$ $v_{k}(b)$ for $i \neq k$, and $v_{k}(a)=v_{k}(b)$ with $a^{\prime}=b^{\prime}$ in $g r_{v_{k}}(K)$. Hence, $y(a)=v_{k}(b)$ and $a^{\prime} \in g r_{y}(K)$ maps to $\left(0, \ldots, 0, b^{\prime}, 0 \ldots, 0\right) \in g r_{v_{1}}(K) \times \ldots \times g r_{v_{n}}(K)\left(b^{\prime}\right.$ in the $k$-th position). Since these $n$-tuples span $g r_{v_{1}}(K) \times \ldots \times g r_{v_{n}}(K)$, it follows that the natural map (1.10) is onto.

Proof of Claim. Assume for simplicity that $\Gamma$ is the divisible hull of $\Gamma_{F}\left(=\Gamma_{F, v}\right.$, the value group of $v$ on $F$ ). For any valuation $z$ on $F$ which is coarser than $v$ there is an associated convex subgroup $\Delta_{F} \subseteq \Gamma_{F}$, which is the kernel of the canonical epimorphism $\Gamma_{F} \rightarrow \Gamma_{F, z}$. Let $\Delta$ be the divisible hull of $\Delta_{F}$ in $\Gamma$, and let $\Lambda=\Gamma / \Delta$, which is a divisible group with ordering inherited from $\Gamma$. Since $\Gamma_{F} \cap \Delta=\Delta_{F}$, the order-preserving inclusion $\Gamma_{F} / \Delta_{F} \hookrightarrow \Lambda$ identifies $\Gamma_{F, z}$ canonically with a subgroup 
of the divisible group $\Lambda$. Likewise, the value group of every extension of $z$ to $K$ can be viewed as a subgroup of $\Lambda$.

For each pair of valuations $v_{i}, v_{j}$ on $K$ with $i \neq j$ there is a valuation $v_{i j}$ on $K$ which is the finest common coarsening of $v_{i}$ and $v_{j}$. ( $v_{i j}$ is the valuation associated to the valuation ring $V_{i} V_{j}$.) Let $\Delta_{i j} \subseteq \Gamma$ be the divisible hull of the convex subgroup of $\Gamma_{F}$ associated to the restriction of $v_{i j}$ to $F$. Let $\Gamma_{i}=\Gamma_{K, v_{i}}$ for $1 \leq i \leq n$. We say that an $n$-tuple $\left(\beta_{1}, \ldots, \beta_{n}\right) \in \Gamma_{1} \times \ldots \times \Gamma_{n}$ is compatible if $\beta_{i}-\beta_{j} \in \Delta_{i j}$ for all $i \neq j$. (This is equivalent to the definition of compatibility in [R, p. 127], though stated a little differently.) For our fixed $b \in K^{\times}$, let $\gamma_{i}=v_{i}(b) \in \Gamma_{i}$, and note that $\left(\gamma_{1}, \ldots, \gamma_{n}\right)$ is compatible since $v_{i}(b)$ and $v_{j}(b)$ have the same image $v_{i j}(b)$ in $\Gamma / \Delta_{i j}$. For our fixed $k$ and for each $i$, let $\epsilon_{i}=\gamma_{k}-\gamma_{i}$. So, for all $i \neq j$, $\epsilon_{i}-\epsilon_{j}=\gamma_{j}-\gamma_{i} \in \Delta_{i j}$. Since $0 \leq|| \epsilon_{i}|-| \epsilon_{j}|| \leq\left|\epsilon_{i}-\epsilon_{j}\right|$ and $\Delta_{i j}$ is convex, we have $\left|\epsilon_{i}\right|-\left|\epsilon_{j}\right| \in \Delta_{i j}$. Because each $\Gamma / \Gamma_{i}$ is a torsion group, there is a positive integer $m$ such that $m\left|\epsilon_{i}\right| \in \Gamma_{i}$ for each $i$. Let $\delta_{i}=\gamma_{i}+m\left|\epsilon_{i}\right| \in \Gamma_{i}$. Then $\delta_{k}=\gamma_{k}$, and for each $i$ we have

$$
\delta_{i}=\gamma_{i}+m\left|\epsilon_{i}\right| \geq \gamma_{i}+\epsilon_{i}=\gamma_{k} .
$$

Note that $\left(\delta_{1}, \ldots, \delta_{n}\right)$ is compatible, since $\left(\gamma_{1}, \ldots, \gamma_{n}\right)$ and $\left(m\left|\epsilon_{1}\right|, \ldots, m\left|\epsilon_{n}\right|\right)$ are compatible. Since the valuations $v_{1}, \ldots, v_{n}$ are incomparable, by [R, Th. 1, p. 135] there is $c \in K^{\times}$with $v_{i}(c)=\delta_{i}$ for each $i$. This $c$ has the properties of the claim.

Let $v_{1}, \ldots, v_{r}$ be all the extensions of $v$ to $K$. For $i=1, \ldots, r$, let $e_{i}=e\left(v_{i} / v\right)$ be the ramification index and $f_{i}=f\left(v_{i} / v\right)$ be the residue degree. We say that the fundamental equality holds for $K / F$ if $[K: F]=\sum_{i=1}^{r} e_{i} f_{i}$.

Corollary 1.9. There is an $F$-gauge on $K$ if and only if the fundamental equality holds for $K / F$. When that condition holds, the F-gauge on $K$ is unique and is defined by

$$
y(x)=\min _{1 \leq i \leq r}\left(v_{i}(x)\right) \quad \text { for } x \in K,
$$

where $v_{1}, \ldots, v_{r}$ are all the extensions of $v$ to $K$.

Proof. Suppose $y$ is an $F$-gauge on $K$. By Prop. 1.8, we may find some extensions $v_{1}, \ldots, v_{n}$ of $v$ to $K$ such that $g r_{y}(K) \cong g r_{v_{1}}(K) \times \ldots \times g r_{v_{n}}(K)$. Now, $\left[\operatorname{gr}_{v_{i}}(K): \operatorname{gr}(F)\right]=e_{i} f_{i}$ by $\left[\mathrm{Bl}_{1}\right.$, Cor. 2], and $\left[\operatorname{gr}_{y}(K): \operatorname{gr}(F)\right]=[K: F]$ since $y$ is an $F$-norm, so

$$
[K: F]=\sum_{i=1}^{n} e_{i} f_{i}
$$

This implies $v_{1}, \ldots, v_{n}$ is the set of all extensions of $v$ to $K$ by [EP, Th. 3.3.4], and the fundamental equality holds. Conversely, if the fundamental equality holds, then formula (1.11) defines an $F$-gauge on $K$.

The following special case will be used in $\$ 1.2$

Corollary 1.10. Let $K / F$ be a finite-degree field extension and let $v$ be a valuation on $F$. Suppose char $(\bar{F}) \nmid[K: F]$. If $v$ extends uniquely to $K$, this extension is an $F$-gauge on $K$.

Proof. In view of Cor. 1.9, it suffices to show that

$$
[K: F]=[\bar{K}: \bar{F}]\left|\Gamma_{K}: \Gamma_{F}\right|,
$$


which may be regarded as a (weak) version of Ostrowski's theorem. We include a proof for lack of a convenient reference. The proof is by reduction to the Henselian case.

Because $\operatorname{char}(F) \nmid[K: F], K$ lies in the separable closure $F_{\text {sep }}$ of $F$. Let $G=$ $\mathcal{G}\left(F_{\text {sep }} / F\right)$, and let $H=\mathcal{G}\left(F_{\text {sep }} / K\right) \subseteq G$. Fix any extension $w$ of $v$ to $F_{\text {sep }}$, and let $Z=\{\sigma \in G \mid w \circ \sigma=w\}$, the decomposition group of $w / v$. Let $F^{h}$ be the fixed field $F_{\text {sep }}^{Z}$; so $\left.w\right|_{F^{h}}$ is a Henselization of $v$ (cf. [EP, p. 121]). Likewise, $Z \cap H$ is the decomposition group of $w /\left.w\right|_{K}$, and for $K^{h}=F_{\text {sep }}^{Z \cap H},\left.w\right|_{K^{h}}$ is a Henselization of $\left.w\right|_{K}$. Now, $G$ acts transitively on the set $\mathcal{E}$ of all extensions of $v$ to $F_{\text {sep }}$. But because $\left.w\right|_{K}$ is the unique extension of $v$ to $K$, this $\mathcal{E}$ is also the set of all extensions of $\left.w\right|_{K}$ to $F_{\text {sep }}$; so $H$ also acts transitively on $\mathcal{E}$. Hence, $G=H Z$. Therefore,

$$
\left[K^{h}: F^{h}\right]=|Z:(Z \cap H)|=|H Z: H|=|G: H|=[K: F] .
$$

Let $\delta_{K / F}=[K: F] /\left([\bar{K}: \bar{F}]\left|\Gamma_{K}: \Gamma_{F}\right|\right)$, the defect of $K$ over $F$. Because the Henselization $\left.w\right|_{F^{h}}$ is an immediate extension of $v$ and $\left.w\right|_{K^{h}}$ is an immediate extension of $\left.w\right|_{K}$, equation (1.13) shows that $\delta_{K / F}=\delta_{K^{h} / F^{h}}$. Let $N$ be the normal closure of $K^{h}$ over $F^{h}$. Clearly, $\delta_{K^{h} / F^{h}}=\delta_{N / F^{h}} / \delta_{N / K^{h}}$. By [EP, Cor. 5.3.8], if $\operatorname{char}(\bar{F})=0$, then $\delta_{N / F^{h}}=\delta_{N / K^{h}}=1$, so $\delta_{K^{h} / F^{h}}=1$. But, if $\operatorname{char}(F)=\bar{p} \neq 0$, then $\delta_{N / F^{h}}$ and $\delta_{N / K^{h}}$ are each a power of $\bar{p}$. Hence, $\delta_{K^{h} / F^{h}}=\bar{p}^{m}$ for some integer $m$. By the Fundamental Inequality, $m \geq 0$. But then $\delta_{K^{h} / F^{h}}$ is an integer, so it must divide $\left[K^{h}: F^{h}\right]$, which is prime to $\bar{p}$ by (1.13). Thus, in all cases we have $\delta_{K^{h} / F^{h}}=1$; hence $\delta_{K / F}=1$, which proves (1.12).

We now turn to the general type of commutative semisimple algebras.

Proposition 1.11. Let $K_{1}, \ldots, K_{m}$ be finite-degree field extensions of a field $F$ and $A=K_{1} \times \ldots \times K_{m}$. Let $v$ be a valuation on $F$, let $y$ be a surmultiplicative $v$-value function on $A$, and let $y_{i}=\left.y\right|_{K_{i}}$ for $i=1, \ldots, m$. If $g_{y}(A)$ is graded semisimple, then each $y_{i}$ has the form (1.8) and, for $a=\left(a_{1}, \ldots, a_{n}\right) \in A$,

$$
y(a)=\min _{1 \leq i \leq m}\left(y_{i}\left(a_{i}\right)\right) .
$$

Moreover, there is a canonical graded isomorphism of graded $\operatorname{gr}(F)$-algebras

$$
g r_{y}(A) \cong g g_{y_{1}}\left(K_{1}\right) \times \ldots \times g r_{y_{m}}\left(K_{m}\right) .
$$

There is a $v$-gauge on $A$ if and only if the fundamental equality holds for each $K_{i} / F$. When that condition holds, there is a unique v-gauge $y$ on A, defined by (1.14), where each $y_{i}$ is the unique $v$-gauge on $K_{i}$ as in Cor. 1.9.

Proof. This is immediate from Cor. 1.9 and Prop. 1.6.

1.2. Gauges on division algebras. Consider a finite-dimensional (not necessarily central) division algebra $D$ over $F$. Suppose $w$ is a valuation on $D$ which extends the valuation $v$ on $F$, and consider the canonical homomorphism

$$
\theta_{D}: \Gamma_{D} / \Gamma_{F} \rightarrow \operatorname{Aut}(Z(\bar{D}))
$$

which for $d \in D^{\times}$maps $w(d)+\Gamma_{F}$ to the automorphism $\bar{z} \mapsto \overline{d z d^{-1}}$ (see JW, $(1.6)])$.

Proposition 1.12. With the notation above, the valuation $w$ is an F-gauge on $D$ if and only if

$$
[D: F]=[\bar{D}: \bar{F}]\left|\Gamma_{D}: \Gamma_{F}\right|
$$


When this condition holds, the gauge $w$ is tame if and only if $Z(\bar{D})$ is separable over $\bar{F}$ and $\operatorname{char}(\bar{F}) \nmid\left|\operatorname{ker}\left(\theta_{D}\right)\right|$.

If char $(\bar{F}) \nmid[D: F]$, the following conditions are equivalent:

(i) $w$ is an F-gauge;

(ii) $w$ is a tame F-gauge;

(iii) $v$ extends uniquely to $Z(D)$.

Proof. Since $\operatorname{gr}(D)$ is a graded division algebra, it is graded semisimple. Therefore, $w$ is an $F$-gauge if and only if $[\operatorname{gr}(D): \operatorname{gr}(F)]=[D: F]$. By an easy calculation, cf. $\mathrm{HW}_{2}$, (1.7)] or $\mathrm{Bl}_{2}$, p. 4278], we have

$$
[\operatorname{gr}(D): \operatorname{gr}(F)]=[\bar{D}: \bar{F}]\left|\Gamma_{D}: \Gamma_{F}\right| .
$$

The first statement follows.

To prove the second statement, assume $w$ is a gauge; hence $[\operatorname{gr}(D): \operatorname{gr}(F)]=$ $[D: F]$. Since

$$
[\operatorname{gr}(D): \operatorname{gr}(F)]=[\operatorname{gr}(D): \operatorname{gr}(Z(D))][\operatorname{gr}(Z(D)): \operatorname{gr}(F)]
$$

and

$$
[D: F]=[D: Z(D)][Z(D): F],
$$

and since, by Prop. 1.1

$$
[D: Z(D)] \geq[\operatorname{gr}(D): \operatorname{gr}(Z(D))]
$$

and

$$
[Z(D): F] \geq[\operatorname{gr}(Z(D)): \operatorname{gr}(F)]
$$

it follows that

$$
[D: Z(D)]=[\operatorname{gr}(D): \operatorname{gr}(Z(D))] .
$$

From the definition of $\theta_{D}$, it is clear that $\Gamma_{Z(D)} / \Gamma_{F} \subseteq \operatorname{ker}\left(\theta_{D}\right)$; hence there is an induced map

$$
\bar{\theta}_{D}: \Gamma_{D} / \Gamma_{Z(D)} \rightarrow \operatorname{Aut}(Z(\bar{D})) .
$$

Clearly, $\left|\operatorname{ker}\left(\theta_{D}\right)\right|=\left|\operatorname{ker}\left(\bar{\theta}_{D}\right)\right|\left|\Gamma_{Z(D)}: \Gamma_{F}\right|$. When (1.19) holds, Boulagouaz proved in $\mathrm{Bl}_{2}$, Cor. 4.4] that $Z(\operatorname{gr}(D))=\operatorname{gr}(Z(D))$ if and only if $Z(\bar{D}) / \overline{Z(D)}$ is separable and $\operatorname{char}(\bar{F}) \nmid\left|\operatorname{ker}\left(\bar{\theta}_{D}\right)\right|$. Therefore, the following statements are equivalent:

(a) $Z(\operatorname{gr}(D))=\operatorname{gr}(Z(D)), \overline{Z(D)} / \bar{F}$ is separable and $\operatorname{char}(\bar{F}) \nmid\left|\Gamma_{Z(D)}: \Gamma_{F}\right|$ (i.e., the gauge $w$ is tame);

(b) $Z(\bar{D}) / \bar{F}$ is separable and $\operatorname{char}(\bar{F}) \nmid\left|\operatorname{ker}\left(\theta_{D}\right)\right|$.

The second statement is thus proved.

If $v$ does not extend uniquely to $Z(D)$, then the fundamental inequality for extensions of valuations [B, VI.8.2, Th. 1] yields

$$
[Z(D): F]>[\overline{Z(D)}: \bar{F}]\left|\Gamma_{Z(D)}: \Gamma_{F}\right| ;
$$

hence (1.17) does not hold, and $w$ is not a gauge. This proves (i) $\Rightarrow$ (iii) (without the hypothesis on $\operatorname{char}(\bar{F}))$. For the rest of the proof, assume $\operatorname{char}(\bar{F}) \nmid[D: F]$. Then $Z(\bar{D}) / \bar{F}$ is separable and $\operatorname{char}(\bar{F}) \nmid\left|\operatorname{ker}\left(\theta_{D}\right)\right|$; hence (i) $\Longleftrightarrow$ (ii). Finally, assume (iii). By Cor. 1.10, we have

$$
[Z(D): F]=[\overline{Z(D)}: \bar{F}]\left|\Gamma_{Z(D)}: \Gamma_{F}\right| .
$$


On the other hand, a noncommutative version of Ostrowski's theorem $\mathrm{M}_{1}$, Th. 3] yields

$$
[D: Z(D)]=[\bar{D}: \overline{Z(D)}]\left|\Gamma_{D}: \Gamma_{Z(D)}\right| .
$$

Therefore, (1.17) holds, and (i) follows.

In the case where $D$ is central and $v$ is Henselian, various other characterizations of tame $F$-gauges are given in the following proposition:

Proposition 1.13. With the same notation as above, suppose $F=Z(D)$ and $v$ is Henselian. The following conditions are equivalent:

(i) the valuation $w$ is a tame F-gauge;

(ii) $D$ is split by the maximal tamely ramified extension of $F$;

(iii) either char $(\bar{F})=0$ or the char $(\bar{F})$-primary component of $D$ is split by the maximal unramified extension of $F$;

(iv) $D$ has a maximal subfield which is tamely ramified over $F$.

Proof. By $\left[\mathrm{HW}_{2}\right.$, Prop. 4.3], conditions (ii)-(iv) above are equivalent to: $[D: F]=$ $[\operatorname{gr}(D): \operatorname{gr}(F)]$ and $Z(\operatorname{gr}(D))=\operatorname{gr}(F)$, hence also to (i).

Definition 1.14. As in $\left[\mathrm{HW}_{2}\right.$, a central division algebra $D$ over a Henselian-valued field $F$ is called tame if the equivalent conditions of Prop. 1.13 hold. Note that by Cor. 3.2 below, $w$ is the unique $F$-gauge on $D$ (if any exists).

Example 1.15 (A nontame gauge). Let $F=\mathbb{Q}(x, y)$, the field of rational fractions in two indeterminates over the rationals. The 2 -adic valuation of $\mathbb{Q}$ extends to a valuation $v$ on $F$ with residue field $\mathbb{F}_{2}(x, y)$; see [EP, Cor. 2.2.2]. This valuation further extends to a valuation $w$ on the quaternion algebra $D=(x, y)_{F}$, with residue division algebra $\bar{D}=\mathbb{F}_{2}(x, y)(\sqrt{x}, \sqrt{y})$. The valuation $w$ is an $F$-gauge on $D$ which is not tame.

Example 1.16 (Gauges that are not valuations). Let $D$ be the quaternion division algebra $(-1,-1)_{\mathbb{Q}}$ over the field of rational numbers. This algebra is split by the field $\mathbb{Q}_{3}$ of 3 -adic numbers; hence the 3 -adic valuation $v$ on $\mathbb{Q}$ does not extend to a valuation on $D$, by [C, Th. 1] or $\left[\mathrm{M}_{1}\right.$, proof of Th. 2]. Let $(1, i, j, k)$ be the quaternion base of $D$ with $i^{2}=j^{2}=-1$ and $k=i j=-j i$, and define a $\mathbb{Q}$-norm $y$ on $D$ by

$$
y\left(a_{0}+a_{1} i+a_{2} j+a_{3} k\right)=\min \left(v\left(a_{0}\right), v\left(a_{1}\right), v\left(a_{2}\right), v\left(a_{3}\right)\right) .
$$

(This $y$ is in fact the armature gauge on $D$ with respect to $v$ on $\mathbb{Q}$ and the abelian subgroup of $D^{\times} / \mathbb{Q}^{\times}$generated by the images of $i$ and $j$, as described in $\S 4.2$ below. But we will not use the $\S 4$ results here.) Lemma 1.2 shows that $y$ is a surmultiplicative value function on $D$. We have $\operatorname{gr}(\mathbb{Q})=\mathbb{F}_{3}\left[t, t^{-1}\right]$, where the indeterminate $t$ is the image of 3 . With primes denoting images in $\operatorname{gr}(D)$, we have $i^{\prime 2}=-1^{\prime}, j^{\prime 2}=-1^{\prime}$, and $i^{\prime} j^{\prime}=k^{\prime}=-j^{\prime} i^{\prime}$. Thus, $1^{\prime}, i^{\prime}, j^{\prime}, k^{\prime}$ span a copy of the 4 -dimensional graded simple graded quaternion algebra $(-1,-1)_{\operatorname{gr}(\mathbb{Q})}$. Since $[\operatorname{gr}(D): \operatorname{gr}(\mathbb{Q})]=[D: \mathbb{Q}]=4$, we have

$$
\operatorname{gr}(D)=(-1,-1)_{\operatorname{gr}(\mathbb{Q})} \cong(-1,-1)_{\mathbb{F}_{3}} \otimes_{\mathbb{F}_{3}} \operatorname{gr}(\mathbb{Q}) \cong M_{2}\left(\mathbb{F}_{3}\right)\left[t, t^{-1}\right] .
$$

Thus, $y$ is a tame gauge on $D$ with $D_{0} \cong M_{2}\left(\mathbb{F}_{3}\right)$ and $\Gamma_{D}=\Gamma_{\mathbb{Q}}=\mathbb{Z}$. One can obtain other gauges on $D$ by conjugation, by using Prop. 1.17 below.

The residue ring $D_{0}$ in the preceding example is a simple ring, though not a division ring. Here is an example where the residue is not simple: Let $k$ be any 
field of characteristic not 2 , and let $F=k(x, z)$, where $x$ and $z$ are algebraically independent over $k$. Let $Q$ be the quaternion division algebra $(1+x, z)_{F}$. Let $v$ be the valuation on $F$ obtained by restriction from the standard Henselian valuation on $k((x))((z))$. So, $\Gamma_{F}=\mathbb{Z} \times \mathbb{Z}$ with right-to-left lexicographic ordering, with $v(x)=(1,0)$ and $v(z)=(0,1)$. Again let $(1, i, j, k)$ denote the quaternion base of $Q$ with $i^{2}=1+x, j^{2}=z$, and $k=i j=-j i$. Define an $F$-norm $y$ on $Q$ by, for $a_{0}, \ldots, a_{3} \in F$,

$$
y\left(a_{0}+a_{1} i+a_{2} j+a_{3} k\right)=\min \left(v\left(a_{0}\right), v\left(a_{1}\right), v\left(a_{2}\right)+\left(0, \frac{1}{2}\right), v\left(a_{3}\right)+\left(0, \frac{1}{2}\right)\right) .
$$

This $y$ is the armature gauge of $Q$ with respect to $v$ and the abelian subgroup of $Q^{\times} / F^{\times}$generated by the images of $i$ and $j$. By using results in $\S 4.2$ or by easy direct calculations, one sees that $y$ is a tame gauge on $Q$ with $\operatorname{gr}(Q)$ graded isomorphic to the graded quaternion algebra $\left(1, y^{\prime}\right)_{\operatorname{gr}(F)}$, with $\Gamma_{Q}=\mathbb{Z} \times \frac{1}{2} \mathbb{Z}$, and $Q_{0} \cong k \times k$, which is clearly not simple.

A gauge $y$ on a division ring $D$ is a valuation iff $g r_{y}(D)$ is a graded division ring. When this occurs, the gauge is invariant under conjugation. But for a gauge which is not a valuation, the associated graded ring is not a graded division ring, so it has nonzero homogeneous elements which are not units. Then, conjugation yields different gauges, as the next proposition shows:

Proposition 1.17. Let $(F, v)$ be a valued field and let $y$ be a v-gauge on a central simple $F$-algebra $A$. For any unit $u \in A^{\times}$, the following conditions are equivalent:

(i) $u^{\prime}$ is a unit of $\operatorname{gr}(A)$;

(ii) $y\left(u x u^{-1}\right)=y(x)$ for all $x \in A$.

Proof. (i) $\Rightarrow$ (ii) If $u^{\prime}$ is a unit of $\operatorname{gr}(A)$, then $u^{\prime}$ is not a zero divisor. So, $u^{\prime}\left(u^{-1}\right)^{\prime}=$ $\left(u u^{-1}\right)^{\prime}=1^{\prime}$ in $\operatorname{gr}(A)$ by (1.5). Hence, $\left(u^{-1}\right)^{\prime}=\left(u^{\prime}\right)^{-1}$, which is a unit of $\operatorname{gr}(A)$. It follows by Lemma 1.3 that $y(u)+y\left(u^{-1}\right)=0$ and for any $x \in A$,

$$
y\left(u x u^{-1}\right)=y(u x)+y\left(u^{-1}\right)=y(u)+y(x)+y\left(u^{-1}\right)=y(x) .
$$

(ii) $\Rightarrow$ (i) The surmultiplicativity of $y$ yields

$$
y(u)+y\left(u^{-1}\right) \leq y(1)=0 .
$$

If equality holds here, then (i) follows by Lemma 1.3. Therefore, if (ii) holds and (i) does not, then for all $x \in A$,

$$
y\left(u x u^{-1}\right)=y(x)>y(u)+y(x)+y\left(u^{-1}\right) .
$$

By (1.5), it follows that $u^{\prime} x^{\prime}\left(u^{-1}\right)^{\prime}=0$ for all $x \in A$; hence $u^{\prime} \operatorname{gr}(A)\left(u^{-1}\right)^{\prime}=\{0\}$ since $\operatorname{gr}(A)$ is spanned by its homogeneous elements. This equation shows that $\operatorname{gr}(A) u^{\prime} \operatorname{gr}(A) \neq \operatorname{gr}(A)$ since $\left(u^{-1}\right)^{\prime} \neq 0$. On the other hand, $\operatorname{gr}(A) u^{\prime} \operatorname{gr}(A) \neq\{0\}$ since $u^{\prime} \neq 0$; hence the 2-sided homogeneous ideal $\operatorname{gr}(A) u^{\prime} \operatorname{gr}(A)$ is not trivial, and $\operatorname{gr}(A)$ is not graded simple. This is a contradiction to Cor. 3.7 below. (Observe that Prop. 1.17 is not used in the sequel; thus, the argument is not circular.)

1.3. Gauges on endomorphism algebras. Let $D$ be a finite-dimensional division algebra over a field $F$, let $M$ be a finite-dimensional right $D$-vector space, and let $A=\operatorname{End}_{D}(M)$. Suppose $w: D \rightarrow \Gamma \cup\{\infty\}$ is a valuation on $D$, and let $v=\left.w\right|_{F}$. Let $\alpha$ be a $D$-norm on $M$. 
Lemma 1.18. Let $\left(m_{i}\right)_{1 \leq i \leq k}$ be a splitting base of $M$ for $\alpha$. For every $f \in A$ and nonzero $m \in M$,

$$
\alpha(f(m))-\alpha(m) \geq \min _{1 \leq i \leq k}\left(\alpha\left(f\left(m_{i}\right)\right)-\alpha\left(m_{i}\right)\right) .
$$

Proof. Let $m=\sum_{i=1}^{k} m_{i} d_{i}$ with $d_{i} \in D$. Then

$$
\alpha(f(m))=\alpha\left(\sum_{i=1}^{k} f\left(m_{i}\right) d_{i}\right) \geq \min _{1 \leq i \leq k}\left(\alpha\left(f\left(m_{i}\right)\right)+w\left(d_{i}\right)\right) .
$$

Writing $\alpha\left(f\left(m_{i}\right)\right)+w\left(d_{i}\right)=\alpha\left(f\left(m_{i}\right)\right)-\alpha\left(m_{i}\right)+\alpha\left(m_{i}\right)+w\left(d_{i}\right)$, we obtain

$\min _{1 \leq i \leq k}\left(\alpha\left(f\left(m_{i}\right)\right)+w\left(d_{i}\right)\right) \geq \min _{1 \leq i \leq k}\left(\alpha\left(f\left(m_{i}\right)\right)-\alpha\left(m_{i}\right)\right)+\min _{1 \leq i \leq k}\left(\alpha\left(m_{i}\right)+w\left(d_{i}\right)\right)$.

Since $\left(m_{i}\right)_{1 \leq i \leq k}$ is a splitting base of $M$, the second term on the right side is $\alpha(m)$. The lemma then follows from (1.20) and (1.21).

In view of the lemma, we may define a function $y_{\alpha}: A \rightarrow \Gamma \cup\{\infty\}$ as follows: for $f \in A$,

$$
y_{\alpha}(f)=\min _{m \in M, m \neq 0}(\alpha(f(m))-\alpha(m)) .
$$

Indeed, the lemma shows that $y_{\alpha}(f)=\min _{1 \leq i \leq k}\left(\alpha\left(f\left(m_{i}\right)\right)-\alpha\left(m_{i}\right)\right)$ for any splitting base $\left(m_{i}\right)_{1 \leq i \leq k}$ of $M$. If $f\left(m_{j}\right)=\sum_{i=1}^{k} m_{i} d_{i j}$ with $d_{i j} \in D$, we have

$$
y_{\alpha}(f)=\min _{1 \leq i, j \leq k}\left(\alpha\left(m_{i}\right)+w\left(d_{i j}\right)-\alpha\left(m_{j}\right)\right) .
$$

Now, let $E=\operatorname{End}_{\operatorname{gr}(D)}(\operatorname{gr}(M))$. Recall that $E$ is graded as follows: for $\gamma \in \Gamma$,

$$
E_{\gamma}=\left\{f \in E \mid f\left(M_{\delta}\right) \subseteq M_{\delta+\gamma} \text { for all } \delta \in \Gamma\right\} .
$$

Proposition 1.19. The map $y_{\alpha}$ of (1.22) is a surmultiplicative $F$-value function on $A$, and there is a canonical $\operatorname{gr}(F)$-algebra isomorphism $\operatorname{gr}(A) \cong_{g} E$. Moreover, $y_{\alpha}$ is an F-gauge (resp. a tame F-gauge) on $A$ if and only if $w$ is an F-gauge (resp. a tame $F$-gauge) on $D$.

Proof. We omit the easy proof that $y_{\alpha}$ is a surmultiplicative $F$-value function on $A$. To define the canonical isomorphism $\operatorname{gr}(A) \rightarrow E$, take any $f \in A$ with $f \neq 0$, and let $\gamma=y_{\alpha}(f) \in \Gamma$. The definition of $y_{\alpha}$ says that $\alpha(f(m)) \geq \alpha(m)+\gamma$ for all $m \in M$, and equality holds for some nonzero $m$. For any $\delta \in \Gamma$, this shows $f$ maps $M^{\geq \delta}$ into $M^{\geq \delta+\gamma}$ and $M^{>\delta}$ into $M^{>\delta+\gamma}$; so, $f$ induces a well-defined additive group homomorphism

$$
\widetilde{f}_{\delta}: M_{\delta} \rightarrow M_{\delta+\gamma}, \quad m^{\prime} \mapsto f(m)+M^{>\delta+\gamma} \quad \text { for all } m \in M \text { with } \alpha(m)=\delta .
$$

Define $\tilde{f}=\bigoplus_{\delta \in \Gamma} \widetilde{f}_{\delta}: \operatorname{gr}(M) \rightarrow \operatorname{gr}(M)$, an additive group homomorphism which shifts graded components by $\gamma$. For any $d \in D^{\times}$and nonzero $m \in M$, we have

$$
\widetilde{f}_{\alpha(m)+w(d)}\left(m^{\prime} \cdot d^{\prime}\right)=\widetilde{f}_{\alpha}\left(m^{\prime}\right) \cdot d^{\prime} \in M_{\alpha(m)+w(d)+\gamma} .
$$

Thus, $\tilde{f} \in E_{\gamma}$, and the definition of $y_{\alpha}$ assures that $\tilde{f} \neq 0$. Since for any $g \in A^{>\gamma}$ we have $\widetilde{f+g}=\widetilde{f}$, there is a well-defined injective map $A_{\gamma} \rightarrow E_{\gamma}$ given by $f+A^{>\gamma} \mapsto$ 
$\widetilde{f}$. The direct sum of these maps is a graded (i.e., grade-preserving) $\operatorname{gr}(F)$-algebra homomorphism $\rho: \operatorname{gr}(A) \rightarrow E$; this map is injective, since it is injective on each homogeneous component of $\operatorname{gr}(A)$. To prove $\rho$ is onto, let $\left(m_{i}\right)_{1 \leq i \leq k}$ be a splitting base of $M$ with respect to $\alpha$; so, $\left(m_{i}^{\prime}\right)_{1 \leq i \leq k}$ is a homogeneous $\operatorname{gr}(D)$-base of $\operatorname{gr}(M)$. If we fix any $i, j$ with $1 \leq i, j \leq k$ and any $d \in D^{\times}$, and define $g \in A$ by $g\left(m_{j}\right)=m_{i} d$ and $g\left(m_{\ell}\right)=0$ for $\ell \neq j$, then $y_{\alpha}(g)=w(d)+\alpha\left(m_{i}\right)-\alpha\left(m_{j}\right)$; so, $\widetilde{g}\left(m_{j}^{\prime}\right)=\left(m_{i} d\right)+A^{>w(d)+\alpha\left(m_{i}\right)-\alpha\left(m_{j}\right)+\alpha\left(m_{j}\right)}=m_{i}^{\prime} \cdot d^{\prime} \in M_{w(d)+\alpha\left(m_{i}\right)}$ and $\widetilde{g}\left(m_{\ell}^{\prime}\right)=0$ for $\ell \neq j$. Since such maps generate $E$ as an additive group, $\rho$ is onto, hence an isomorphism, as desired.

It follows from the isomorphism $\operatorname{gr}(A) \cong_{g} E$ that $\operatorname{gr}(A)$ is a graded simple $\operatorname{gr}(F)$ algebra. Thus, $y_{\alpha}$ is an $F$-gauge on $A$ iff $y_{\alpha}$ is an $F$-norm on $A$, iff, by Prop. 1.1(ii), $[\operatorname{gr}(A): \operatorname{gr}(F)]=[A: F]$. We have

$$
[A: F]=k^{2}[D: F] \quad \text { and } \quad[\operatorname{gr}(A): \operatorname{gr}(F)]=[E: \operatorname{gr}(F)]=k^{2}[\operatorname{gr}(D): \operatorname{gr}(F)] .
$$

Thus, $y_{\alpha}$ is an $F$-norm on $A$ iff $[\operatorname{gr}(D): \operatorname{gr}(F)]=[D: F]$ iff, by (1.18) and Prop. 1.12, $w$ is an $F$-gauge on $D$.

Since $Z(A)=Z(D)$ (up to canonical isomorphism) with $\left.y_{\alpha}\right|_{Z(A)}=\left.w\right|_{Z(D)}$ and $Z(\operatorname{gr}(A)) \cong_{g} Z(\operatorname{gr}(D))$ (a $\operatorname{gr}(F)$-algebra isomorphism), $y_{\alpha}$ is a tame $F$-gauge on $A$ iff $w$ is a tame $F$-gauge on $D$.

We now compare the "gauge ring" $V_{A}=\left\{f \in A \mid y_{\alpha}(f) \geq 0\right\}$ with the valuation rings $V_{D}$ and $V_{F}$.

Lemma 1.20. The following conditions are equivalent:

(i) $V_{A}$ is integral over $V_{F}$;

(ii) $V_{D}$ is integral over $V_{F}$;

(iii) the valuation $v$ on $F$ has a unique extension to $Z(D)$.

In particular, these conditions all hold if $v$ is Henselian.

Proof. (i) $\Longleftrightarrow$ (ii) Let $\left(m_{i}\right)_{1 \leq i \leq k}$ be a splitting base of $M$ with respect to $\alpha$, and let $f \in A$. If $f\left(m_{j}\right)=\sum_{i=1}^{k} m_{i} d_{i j}$, then (1.23) shows that $f \in V_{A}$ iff $w\left(d_{i j}\right) \geq$ $\alpha\left(m_{i}\right)-\alpha\left(m_{j}\right)$ for all $i, j$. Let $e_{1}, \ldots, e_{k}$ be the orthogonal idempotents of $A$ for the splitting base $\left(m_{i}\right)_{1 \leq i \leq k}$ of $M$, i.e., $e_{i}\left(m_{j}\right)=\delta_{i j} m_{i}$ (Kronecker delta). We have $y_{\alpha}\left(e_{i}\right)=0$, so each $e_{i} \in V_{A}$. For $f$ with matrix $\left(d_{i j}\right)$ as above, $e_{i} f e_{i}$ has a matrix with $i i$-entry $d_{i i}$ and all other entries 0 . Hence, $e_{i} V_{A} e_{i} \cong V_{D}$, a $V_{F}$-algebra isomorphism. If $V_{A}$ is integral over $V_{F}$, then $V_{D} \cong e_{1} V_{A} e_{1}$ must also be integral over $V_{F}$. Conversely, suppose $V_{D}$ is integral over $V_{F}$. Then, each $e_{i} V_{A} e_{i}$ is integral over $V_{F}$, since it is isomorphic to $V_{D}$. For $j \neq i$, each element of $e_{i} V_{A} e_{j}$ has square 0 , so is integral over $V_{F}$. Since $\sum_{i=1}^{k} e_{i}=1$, we have $V_{A}=\sum_{1 \leq i, j \leq k} e_{i} V_{A} e_{j}$, with the elements of each summand integral over $V_{F}$. Because $A$ is a p.i.-algebra over $F$, the theorem [AS, Th. 2.3] of Amitsur and Small shows that $V_{A}$ is integral over $V_{F}$.

(ii) $\Longleftrightarrow$ (iii) Let $Z=Z(D)$, the center of $D$, and let $V_{Z}$ be the valuation ring of $\left.w\right|_{Z}$. Then $V_{D}$ is always integral over $V_{Z}$, by $\mathrm{W}_{1}$, Cor.], and $V_{Z}$ is integral over $V_{F}$ iff $\left.w\right|_{Z}$ is the unique extension of the valuation $v$ to $Z$; see [B] Ch. VI, $\S 8.3$, Remark] or [EP, Cor. 3.1.4].

Remark 1.21. The value function $y_{\alpha}$ on $\operatorname{End}_{D}(M)$ associated to a norm $\alpha$ on $M$ is defined by Bruhat and Tits in [BT, 1.11, 1.13], where it is denoted by End $\alpha$ and 
called a norme carrée. In [BT, 2.13, Cor.] (see also the Appendix of [BT]), Bruhat and Tits establish a bijection between the set of normes carrées on $\operatorname{End}_{D}(M)$ and the building of $G L(M)$, when the rank of the ordered group $\Gamma$ is 1 .

The following result is in $\mathrm{BT}, 1.13]$ :

Proposition 1.22. For $D$-norms $\alpha, \beta$ on $M$, the following conditions are equivalent:

(i) $\alpha-\beta$ is constant; i.e. there exists $\gamma \in \Gamma$ such that $\alpha(m)=\beta(m)+\gamma$ for all $m \in M$

(ii) $y_{\alpha}=y_{\beta}$.

Proof. (i) $\Rightarrow$ (ii) is clear from the definition (1.22). To prove (ii) $\Rightarrow$ (i), choose any $m, n \in M$ with $m \neq 0$ and let $A(m, n)=\{f \in A \mid f(m)=n\}$. By definition of $y_{\alpha}$, we have

$$
y_{\alpha}(f) \leq \alpha(n)-\alpha(m) \quad \text { for } f \in A(m, n) .
$$

On the other hand, we may choose a splitting base $\left(m_{i}\right)_{1 \leq i \leq k}$ of $M$ with $m_{1}=m$ (see [RTW, Prop. 2.5]) and define $g \in A(m, n)$ by $g\left(m_{1}\right)=n, g\left(m_{i}\right)=0$ for $i>1$; then $y_{\alpha}(g)=\alpha(n)-\alpha(m)$. Therefore,

$$
\alpha(n)-\alpha(m)=\max _{g \in A(m, n)}\left(y_{\alpha}(g)\right) .
$$

If (ii) holds, it follows from (1.24) that

$$
\alpha(n)-\alpha(m)=\beta(n)-\beta(m) \quad \text { for all } m, n \in M \text { with } m \neq 0 .
$$

Condition (i) readily follows.

1.4. Gauges on tensor products. If $P$ and $Q$ are two graded vector spaces over a graded field $K$, then the grading on $P \otimes_{K} Q$ is given by $\left(P \otimes_{K} Q\right)_{\gamma}=$ $\sum_{\delta \in \Gamma} P_{\delta} \otimes_{K_{0}} Q_{\gamma-\delta}$

Proposition 1.23. Let $(F, v)$ be a valued field, and let $M$ and $N$ be $F$-vector spaces such that $M$ has an $F$-norm $\alpha$ and $N$ has an $F$-value function $\beta$. There is a unique $F$-value function $t$ on $M \otimes_{F} N$ such that there is a graded isomorphism of $\operatorname{gr}(F)$-vector spaces

$$
\operatorname{gr}_{t}\left(M \otimes_{F} N\right) \cong_{g} \operatorname{gr}_{\alpha}(M) \otimes_{g r(F)} \operatorname{gr}_{\beta}(N)
$$

satisfying $(m \otimes n)^{\prime} \mapsto m^{\prime} \otimes n^{\prime}$ for all $m \in M$ and $n \in N$. So, $t(m \otimes n)=\alpha(m)+\beta(n)$. If $\beta$ is an $F$-norm, then $t$ is also an F-norm.

The unique value function $t$ on $M \otimes_{F} N$ satisfying the condition in the proposition will be denoted $\alpha \otimes \beta$, and the canonical isomorphism of graded vector spaces will be viewed as an identification.

The following lemma will be used in the proof of Prop. 1.23,

Lemma 1.24. Let $D$ be a division ring with a valuation $w$, and let $P$ be a right $D$ vector space. Let $u$ and $t$ be $D$-value functions $P \rightarrow \Gamma \cup\{\infty\}$ such that $t(p) \leq u(p)$ for all $p \in P$. Then, there is a canonical induced $\operatorname{gr}(D)$-vector space homomorphism $\chi_{t, u}: g r_{t}(P) \rightarrow g r_{u}(P)$ which is injective iff $t=u$.

Proof. Clear. 
Proof of Proposition 1.23. Let $\left(m_{i}\right)_{1 \leq i \leq k}$ be a splitting base of $M$ with respect to $\alpha$. Define $t: M \otimes_{F} N \rightarrow \Gamma \cup\{\infty\}$ by

$$
t\left(\sum_{i=1}^{k} m_{i} \otimes n_{i}\right)=\min _{1 \leq i \leq k}\left(\alpha\left(m_{i}\right)+\beta\left(n_{i}\right)\right) \quad \text { for any } n_{i} \in N .
$$

Clearly, $t$ is an $F$-value function on $M \otimes_{F} N$, and

$$
\left(M \otimes_{F} N\right)^{\geq \gamma}=\bigoplus_{i=1}^{k} m_{i} \otimes N \geq \gamma-\alpha\left(m_{i}\right) \quad \text { for } \gamma \in \Gamma .
$$

Also, since $\left(m_{i}^{\prime}\right)_{1 \leq i \leq k}$ is a homogeneous $\operatorname{gr}(F)$-base of $\operatorname{gr}(M)$, we have

$$
\left(\operatorname{gr}(M) \otimes_{\operatorname{gr}(F)} \operatorname{gr}(N)\right)_{\gamma}=\bigoplus_{i=1}^{k} m_{i}^{\prime} \otimes N_{\gamma-\alpha\left(m_{i}\right)} .
$$

For $\gamma \in \Gamma$, let $\pi_{\gamma}: N \geq \gamma \rightarrow N_{\gamma}$ be the canonical map. Define a surjective map $\psi_{\gamma}:\left(M \otimes_{F} N\right)^{\geq \gamma} \rightarrow\left(\operatorname{gr}(M) \otimes_{\operatorname{gr}(F)} \operatorname{gr}(N)\right)_{\gamma}$ as follows:

$$
\psi_{\gamma}\left(\sum_{i=1}^{k} m_{i} \otimes n_{i}\right)=\sum_{i=1}^{k} m_{i}^{\prime} \otimes \pi_{\gamma-\alpha\left(m_{i}\right)}\left(n_{i}\right) \quad \text { for } n_{i} \in N^{\geq \gamma-\alpha\left(m_{i}\right)} .
$$

This $\psi_{\gamma}$ is clearly an additive group homomorphism. Moreover, $\psi_{\gamma}\left(\sum m_{i} \otimes n_{i}\right)=0$ iff each $n_{i} \in N^{>\gamma-\alpha\left(m_{i}\right)}$ iff $\sum m_{i} \otimes n_{i} \in\left(M \otimes_{F} N\right)^{>\gamma}$. Thus, $\psi_{\gamma}$ induces a group isomorphism $\varphi_{\gamma}:\left(M \otimes_{F} N\right)_{\gamma} \rightarrow\left(\operatorname{gr}(M) \otimes_{\operatorname{gr}(F)} \operatorname{gr}(N)\right)_{\gamma}$ and $\varphi=\bigoplus_{\gamma \in \Gamma} \varphi_{\gamma}$ is a graded $\operatorname{gr}(F)$-vector space isomorphism $g r_{t}\left(M \otimes_{F} N\right) \rightarrow g r_{\alpha}(M) \otimes_{g r_{v}}(F) \operatorname{gr}_{\beta}(N)$. To see that $\varphi\left((m \otimes n)^{\prime}\right)=m^{\prime} \otimes n^{\prime}$ for $m \in M$ and $n \in N$, let $m=\sum_{i=1}^{k} m_{i} r_{i}$ with $r_{i} \in F$.

Then $m \otimes n=\sum_{i=1}^{k} m_{i} \otimes r_{i} n$ and $\alpha(m)=\min \left\{\alpha\left(m_{i}\right)+v\left(r_{i}\right)\right\}$, so

$$
t(m \otimes n)=\min _{1 \leq i \leq k}\left(\alpha\left(m_{i}\right)+\beta(n)+v\left(r_{i}\right)\right)=\alpha(m)+\beta(n)
$$

and

$$
\varphi\left((m \otimes n)^{\prime}\right)=\psi_{\alpha(m)+\beta(n)}\left(\sum_{i=1}^{k} m_{i} \otimes r_{i} n\right)=\sum_{i=1}^{k} m_{i}^{\prime} \otimes \pi_{\alpha(m)-\alpha\left(m_{i}\right)+\beta(n)}\left(r_{i} n\right) .
$$

Note that

$$
\pi_{\alpha(m)-\alpha\left(m_{i}\right)+\beta(n)}\left(r_{i} n\right)= \begin{cases}r_{i}^{\prime} n^{\prime} & \text { if } \alpha\left(m_{i}\right)+v\left(r_{i}\right)=\alpha(m), \\ 0 & \text { if } \alpha\left(m_{i}\right)+v\left(r_{i}\right)>\alpha(m) .\end{cases}
$$

Changing the indexing if necessary, we may assume $\alpha\left(m_{i}\right)+v\left(r_{i}\right)=\alpha(m)$ for $i=1$, $\ldots, \ell$ and $\alpha\left(m_{i}\right)+v\left(r_{i}\right)>\alpha(m)$ for $i>\ell$. Then $m^{\prime}=\sum_{i=1}^{\ell} m_{i}^{\prime} r_{i}^{\prime}$ and

$$
\varphi\left((m \otimes n)^{\prime}\right)=\sum_{i=1}^{\ell} m_{i}^{\prime} \otimes r_{i}^{\prime} n^{\prime}=m^{\prime} \otimes n^{\prime},
$$

as desired.

Now, suppose $u: M \otimes_{F} N \rightarrow \Gamma \cup\{\infty\}$ is an $F$-value function with the same property. For $m \in M$ and $n \in N$ we have $u(m \otimes n)=\alpha(m)+\beta(n)$, since the degree 
of $(m \otimes n)^{\prime}$ in $\operatorname{gr}_{u}(M \otimes N)$ is the same as the degree of $m^{\prime} \otimes n^{\prime}$ in $\operatorname{gr}_{\alpha}(M) \otimes \operatorname{gr}_{\beta}(N)$. Therefore, for $n_{i} \in N$ we have

$u\left(\sum_{i=1}^{k} m_{i} \otimes n_{i}\right) \geq \min _{1 \leq i \leq k}\left(u\left(m_{i} \otimes n_{i}\right)\right)=\min _{1 \leq i \leq k}\left(\alpha\left(m_{i}\right)+\beta\left(n_{i}\right)\right)=t\left(\sum_{i=1}^{k} m_{i} \otimes n_{i}\right)$.

We have the graded $\operatorname{gr}(F)$-vector space isomorphisms $\varphi_{t}: \operatorname{gr}_{t}\left(M \otimes_{F} N\right) \rightarrow$ $g r_{\alpha}(M) \otimes_{g r(F)} g r_{\beta}(N)$ and $\varphi_{u}: g r_{u}\left(M \otimes_{F} N\right) \rightarrow g r_{\alpha}(M) \otimes_{g r(F)} g r_{\beta}(N)$. Because of the inequality in (1.26), Lemma 1.24 yields a canonical $\operatorname{gr}(F)$-vector space homomorphism $\chi_{t, u}: \operatorname{gr}_{t}\left(M \otimes_{F} N\right) \rightarrow g r_{u}\left(M \otimes_{F} N\right)$. Our hypotheses on $\varphi_{t}$ and $\varphi_{u}$ imply that $\varphi_{u} \circ \chi_{t, u}$ and $\varphi_{t}$ agree on $(m \otimes n)^{\prime}$ for all $m \in M$ and $n \in N$. Since such $(m \otimes n)^{\prime}$ form a generating set for $g r_{t}\left(M \otimes_{F} N\right)$, we have $\varphi_{u} \circ \chi_{t, u}=\varphi_{t}$. Then, $\chi_{t, u}$ is an isomorphism, since $\varphi_{t}$ and $\varphi_{u}$ are each isomorphisms. Lemma 1.24 then shows that $u=t$, proving the desired uniqueness of $t$.

If $\beta$ is an $F$-norm on $N$, say with splitting base $\left(n_{i}\right)_{1 \leq i \leq \ell}$, then it follows easily from (1.25) that $\left(m_{i} \otimes n_{j}\right)_{\substack{1<i<k \\ 1<j<\ell}}$ is a splitting base for $t$ on $M \otimes_{F} N$, so $t$ is an $F$-norm for $M \otimes_{F} N$.

Remark 1.25. A basis-free description of $\alpha \otimes \beta$ is stated in [BT, p. 269]: for $s \in$ $M \otimes N$,

$$
\alpha \otimes \beta(s)=\sup \left\{\min _{1 \leq j \leq \ell}\left(\alpha\left(p_{j}\right)+\beta\left(q_{j}\right)\right) \mid s=\sum_{j=1}^{\ell} p_{j} \otimes q_{j}\right\} .
$$

To see this equality, for $s \in M \otimes_{F} N$, let

$$
u(s)=\sup \left\{\min _{1 \leq j \leq \ell}\left(\alpha\left(p_{j}\right)+\beta\left(q_{j}\right)\right) \mid s=\sum_{j=1}^{\ell} p_{j} \otimes q_{j}\right\} .
$$

Take any representation for $s$ as $\sum_{j=1}^{\ell} p_{j} \otimes q_{j}$. Write each $p_{j}=\sum_{i=1}^{k} m_{i} r_{i j}$ with $r_{i j} \in F$.

Then, $s=\sum_{i=1}^{k} m_{i} \otimes\left(\sum_{j=1}^{\ell} r_{i j} q_{j}\right)$, and

$$
\begin{aligned}
\min _{1 \leq j \leq \ell}\left(\alpha\left(p_{j}\right)+\beta\left(q_{j}\right)\right) & =\min _{1 \leq j \leq \ell}\left(\alpha\left(\sum_{i=1}^{k} m_{i} r_{i j}\right)+\beta\left(q_{j}\right)\right) \\
& =\min _{1 \leq j \leq \ell}\left(\left(\min _{1 \leq i \leq k} \alpha\left(m_{i}\right)+v\left(r_{i j}\right)\right)+\beta\left(q_{j}\right)\right) \\
& =\min _{i, j}\left(\alpha\left(m_{i}\right)+v\left(r_{i j}\right)+\beta\left(q_{j}\right)\right) \\
& =\min _{1 \leq i \leq k}\left(\alpha\left(m_{i}\right)+\min _{1 \leq j \leq \ell}\left(v\left(r_{i j}\right)+\beta\left(q_{j}\right)\right)\right. \\
& \leq \min _{1 \leq i \leq k}\left(\alpha\left(m_{i}\right)+\beta\left(\sum_{j=1}^{\ell} r_{i j} q_{j}\right)\right) \\
& =\alpha \otimes \beta\left(\sum_{i=1}^{k} m_{i} \otimes\left(\sum_{j=1}^{\ell} r_{i j} q_{j}\right)\right)=\alpha \otimes \beta(s) .
\end{aligned}
$$


So, $\alpha \otimes \beta(s)$ is an upper bound for the quantities in the description of $u(s)$. But, by using the representation $s=\sum_{i=1}^{k} m_{i} \otimes\left(\sum_{j=1}^{\ell} r_{i j} q_{j}\right)$, we see that $\alpha \otimes \beta(s)$ is one of those quantities. Hence, the sup exists, and $u(s)=\alpha \otimes \beta(s)$.

Corollary 1.26. Suppose $(F, v)$ is a valued field and $A$ is a semisimple $F$-algebra with an $F$-gauge $y$. Let $(L, w)$ be any valued field extending $(F, v)$. Then, $y \otimes w$ is a surmultiplicative $L$-value function on $A \otimes_{F} L$ and the canonical isomorphism

$$
\operatorname{gr}\left(A \otimes_{F} L\right) \cong_{g} \operatorname{gr}(A) \otimes_{\operatorname{gr}(F)} \operatorname{gr}(L)
$$

is an isomorphism of $\operatorname{gr}(L)$-algebras. Moreover, $y \otimes w$ is an L-gauge iff $\operatorname{gr}(A) \otimes_{\operatorname{gr}(F)}$ $\operatorname{gr}(L)$ is semisimple iff $Z(\operatorname{gr}(A)) \otimes_{\operatorname{gr}(F)} \operatorname{gr}(L)$ is a direct sum of graded fields. Furthermore, $y \otimes w$ is a tame L-gauge iff $y$ is a tame F-gauge.

Proof. Let $z=y \otimes w$. Prop. 1.23 shows that $z$ is a well-defined $F$-value function on $A \otimes_{F} L$ with $z(a \otimes \ell)=y(a)+w(\ell)$ for all $a \in A, \ell \in L$. This equation shows that $z$ is actually an $L$-value function. Moreover, if $\left(a_{i}\right)_{1 \leq i \leq k}$ is an $F$-splitting base of $A$ with respect to $y$, then formula (1.25) shows that $\left(a_{i} \otimes 1\right)_{1 \leq i \leq k}$ is an $L$-splitting base of $A \otimes_{F} L$ with respect to $z$. Since

$z\left(\left(a_{i} \otimes 1\right)\left(a_{j} \otimes 1\right)\right)=z\left(a_{i} a_{j} \otimes 1\right)=y\left(a_{i} a_{j}\right) \geq y\left(a_{i}\right)+y\left(a_{j}\right)=z\left(a_{i} \otimes 1\right)+z\left(a_{j} \otimes 1\right)$, Lemma 1.2 shows that $z$ is surmultiplicative. The value-function compatible $F$ algebra homomorphism $A \rightarrow A \otimes_{F} L$ induces a graded $\operatorname{gr}(F)$-algebra homomorphism $\operatorname{gr}(A) \rightarrow \operatorname{gr}\left(A \otimes_{F} L\right)$, and hence a graded $\operatorname{gr}(L)$-algebra homomorphism $\varphi: \operatorname{gr}(A) \otimes_{\operatorname{gr}(F)} \operatorname{gr}(L) \rightarrow \operatorname{gr}\left(A \otimes_{F} L\right)$. This $\varphi$ is bijective, since it coincides with the $\operatorname{gr}(F)$-vector space isomorphism of Prop. 1.23. Since $z$ satisfies all the other conditions for an $L$-gauge, $z$ is an $L$-gauge iff $\operatorname{gr}\left(A \otimes_{F} L\right)$ is a graded semisimple $\operatorname{gr}(L)$-algebra iff $\operatorname{gr}(A) \otimes_{\operatorname{gr}(F)} \operatorname{gr}(L)$ is a graded semisimple $\operatorname{gr}(L)$-algebra. We have $\operatorname{gr}(A) \otimes_{\operatorname{gr}(F)} \operatorname{gr}(L) \cong_{g} \operatorname{gr}(A) \otimes_{Z(\operatorname{gr}(A))}\left(Z(\operatorname{gr}(A)) \otimes_{\operatorname{gr}(F)} \operatorname{gr}(L)\right)$. Since $\operatorname{gr}(A)$ is semisimple, $\operatorname{gr}(A) \otimes_{\operatorname{gr}(F)} \operatorname{gr}(L)$ is semisimple iff $Z(\operatorname{gr}(A)) \otimes_{\operatorname{gr}(F)} \operatorname{gr}(L)$ is a direct sum of graded fields. This is justified just as in the ungraded analogue by reducing to the simple case and using the fact $\left[\mathrm{HW}_{2}\right.$, Prop. 1.1] that if $B$ is a graded central simple algebra over a graded field $K$ and $M$ is any graded field extension of $K$, then $B \otimes_{K} M$ is a graded central simple algebra over $M$.

Applying the first part of the corollary to $Z(A)$ with the gauge $\left.y\right|_{Z(A)}$, we obtain

$$
\operatorname{gr}\left(Z\left(A \otimes_{F} L\right)\right)=\operatorname{gr}\left(Z(A) \otimes_{F} L\right) \cong_{g} \operatorname{gr}(Z(A)) \otimes_{\operatorname{gr}(F)} \operatorname{gr}(L) .
$$

On the other hand,

$$
Z\left(\operatorname{gr}\left(A \otimes_{F} L\right)\right) \cong_{g} Z\left(\operatorname{gr}(A) \otimes_{\operatorname{gr}(F)} \operatorname{gr}(L)\right)=Z(\operatorname{gr}(A)) \otimes_{\operatorname{gr}(F)} \operatorname{gr}(L) ;
$$

hence

$$
[\operatorname{gr}(Z(A \otimes L)): \operatorname{gr}(L)]=[\operatorname{gr}(Z(A)): \operatorname{gr}(F)]
$$

and

$$
[Z(\operatorname{gr}(A \otimes L)): \operatorname{gr}(L)]=[Z(\operatorname{gr}(A)): \operatorname{gr}(F)] .
$$

Since $\operatorname{gr}(Z(A \otimes L)) \subseteq Z(\operatorname{gr}(A \otimes L))$, these finite-dimensional $\operatorname{gr}(L)$-algebras coincide iff they have the same dimension. Similarly, $\operatorname{gr}(Z(A))=Z(\operatorname{gr}(A))$ iff $[\operatorname{gr}(Z(A)): \operatorname{gr}(F)]=[Z(\operatorname{gr}(A)): \operatorname{gr}(F)]$. Therefore, (1.28) and (1.29) show that

$$
\operatorname{gr}(Z(A \otimes L))=Z(\operatorname{gr}(A \otimes L)) \quad \text { iff } \operatorname{gr}(Z(A))=Z(\operatorname{gr}(A)) .
$$


Moreover, since $\operatorname{gr}(L)$ is a free $\operatorname{gr}(F)$-module, it follows from (1.27) that $Z(\operatorname{gr}(A \otimes L))$ is separable over $\operatorname{gr}(L)$ iff $Z(\operatorname{gr}(A))$ is separable over $\operatorname{gr}(F)$, by [KO, Ch. III, Prop. 2.1 and 2.2]. Therefore, $y \otimes w$ is a tame $L$-gauge iff $y$ is a tame $F$-gauge.

Remark 1.27. In the context of Cor. 1.26, since $\operatorname{gr}(A)$ is semisimple, $Z(\operatorname{gr}(A))$ is a direct sum of graded fields finite-dimensional over $\operatorname{gr}(F)$. If each of these graded fields is separable over $\operatorname{gr}(F)$, or if $\operatorname{gr}(L)$ is separable over $\operatorname{gr}(F)$, then $Z(\operatorname{gr}(A)) \otimes_{\operatorname{gr}(F)} \operatorname{gr}(L)$ is a direct sum of graded fields. Recall from $\mathrm{HW}_{1}$, Th. 3.11, Def. 3.4] that if $[\operatorname{gr}(L): \operatorname{gr}(F)]<\infty$, then $\operatorname{gr}(L)$ is separable over $\operatorname{gr}(F)$ iff $\bar{L}$ is separable over $\bar{F}$ and $\operatorname{char}(\bar{F}) \nmid\left|\Gamma_{L}: \Gamma_{F}\right|$.

Corollary 1.28. Suppose $(F, v)$ is a valued field and $A$ and $B$ are semisimple $F$ algebras with respective $F$-gauges $y$ and $z$. If $\operatorname{gr}(A) \otimes_{\operatorname{gr}(F)} \operatorname{gr}(B)$ is graded semisimple, then $y \otimes z$ is an F-gauge on $A \otimes_{F} B$, and the canonical isomorphism

$$
\operatorname{gr}\left(A \otimes_{F} B\right) \cong_{g} \operatorname{gr}(A) \otimes_{\operatorname{gr}(F)} \operatorname{gr}(B)
$$

is an isomorphism of graded $\operatorname{gr}(F)$-algebras. Moreover $\operatorname{gr}(A) \otimes_{\operatorname{gr}(F)} \operatorname{gr}(B)$ is graded semisimple iff $Z(\operatorname{gr}(A)) \otimes_{\operatorname{gr}(F)} Z(\operatorname{gr}(B))$ is a direct sum of graded fields. If $A$ and $B$ are central simple and $y, z$ are tame gauges, then $y \otimes z$ is a tame gauge.

Proof. Since $y$ is an $F$-norm on $A$, say with splitting base $\left(a_{i}\right)_{1 \leq i \leq k}$, and $z$ is an $F$-norm on $B$, say with splitting base $\left(b_{j}\right)_{1 \leq j \leq \ell}$, we saw in the proof of Prop. 1.23 that $\left(a_{i} \otimes b_{j}\right)_{\substack{1 \leq i \leq k \\ 1 \leq j \leq \ell}}$ is a splitting base for the $\bar{F}$-norm $y \otimes z$ on $A \otimes_{F} B$. For any $a_{i}$, $a_{p}, b_{j}, b_{q}$, we have, by Prop. 1.23 .

$$
\begin{gathered}
(y \otimes z)\left(\left(a_{i} \otimes b_{j}\right) \cdot\left(a_{p} \otimes b_{q}\right)\right)=(y \otimes z)\left(a_{i} a_{p} \otimes b_{j} b_{q}\right)=y\left(a_{i} a_{p}\right)+z\left(b_{j} b_{q}\right) \\
\geq y\left(a_{i}\right)+y\left(a_{p}\right)+z\left(b_{j}\right)+z\left(b_{q}\right)=(y \otimes z)\left(a_{i} \otimes b_{j}\right)+(y \otimes z)\left(a_{p} \otimes b_{q}\right) .
\end{gathered}
$$

Therefore, Lemma 1.2 shows that $y \otimes z$ is surmultiplicative. We have $\operatorname{gr}\left(A \otimes_{F} B\right) \cong_{g} \operatorname{gr}(A) \otimes_{\operatorname{gr}(F)} \operatorname{gr}(B)$ by Prop. 1.23. Thus, $y \otimes z$ is an $F$-gauge on $A \otimes_{F} B$ iff $\operatorname{gr}(A) \otimes_{\operatorname{gr}(F)} \operatorname{gr}(B)$ is graded semisimple. Since $\operatorname{gr}(A) \otimes_{\operatorname{gr}(F)} \operatorname{gr}(B) \cong_{g}$ $\operatorname{gr}(A) \otimes_{Z(\operatorname{gr}(A))}\left[Z(\operatorname{gr}(A)) \otimes_{\operatorname{gr}(F)} Z(\operatorname{gr}(B))\right] \otimes_{Z(\operatorname{gr}(B))} \operatorname{gr}(B)$, the desired graded semisimplicity holds iff $Z(\operatorname{gr}(A)) \otimes_{\operatorname{gr}(F)} Z(\operatorname{gr}(B))$ is a direct sum of graded fields. This follows just as in the ungraded case, using $\left[\mathrm{HW}_{1}\right.$, Prop. 1.1].

The last statement is immediate since a graded tensor product of graded central simple $\operatorname{gr}(F)$-algebras is graded central simple over $\operatorname{gr}(F)$, by $\left[\mathrm{HW}_{2}\right.$, Prop. 1.1].

Note that the constructions in $\S \Phi 1.3$ and 1.4 could be done in more generality. For instance, the valuation $w$ in $\$ 1.3$ could be replaced by a gauge, and in $\$ 1.4$ the tensor products could be taken over division algebras instead of fields. Moreover, given a norm $\alpha$ on a right $D$-vector space $M$ one can define a dual norm $\alpha^{*}$ on the left $D$-vector space $M^{*}=\operatorname{Hom}_{D}(M, D)$ and check that the tensor product $\alpha \otimes \alpha^{*}$ corresponds to the gauge $y_{\alpha}$ under the canonical isomorphism $M \otimes_{D} M^{*} \cong$ $\operatorname{End}_{D}(M)$. (See $\left.\left.\underline{\mathrm{BT}}, \S 1\right].\right)$

\section{Graded Central simple algebras}

Let $K$ be a graded field, and let $B$ be a (finite-dimensional) graded central simple $K$-algebra. By the graded version of Wedderburn's theorem, see, e.g., $\mathrm{HW}_{2}$, Prop. 1.3], there is a (finite-dimensional) graded central $K$-division algebra $E$ and 
a finite-dimensional graded right $E$-vector space $N$ such that $B \cong_{g} \operatorname{End}_{E}(N)$. We identify $B$ with $\operatorname{End}_{E}(N)$. The grading on $B$ is given as follows: for any $\epsilon \in \Gamma$,

$$
B_{\epsilon}=\left\{f \in B \mid f\left(N_{\delta}\right) \subseteq N_{\delta+\epsilon} \text { for all } \delta \in \Gamma\right\} .
$$

Since $E$ is a graded division ring, $E_{0}$ is a division ring, and for each $\gamma \in \Gamma_{E}, E_{\gamma}$ is a 1-dimensional left and right $E_{0}$-vector space. The grade set of $N, \Gamma_{N}=\{\gamma \in$ $\left.\Gamma \mid N_{\gamma} \neq\{0\}\right\}$, need not be a group, but it is clearly a union $\Gamma_{N}=\Gamma_{1} \cup \ldots \cup \Gamma_{k}$, where each $\Gamma_{i}$ is a (nonempty) coset of the group $\Gamma_{E}$. Then, $N$ has a canonical direct sum decomposition into graded $E$-vector subspaces

$$
N=\bigoplus_{i=1}^{k} N_{\Gamma_{i}}, \quad \text { where } \quad N_{\Gamma_{i}}=\bigoplus_{\gamma \in \Gamma_{i}} N_{\gamma} .
$$

For each coset $\Gamma_{i}$, choose and fix a representative $\gamma_{i} \in \Gamma_{i}$.

Proposition 2.1. The grade set of $B$ is

$$
\Gamma_{B}=\bigcup_{i, j=1}^{k}\left(\gamma_{i}-\gamma_{j}\right)+\Gamma_{E}
$$

and there is a canonical isomorphism of $K_{0}$-algebras

$$
B_{0} \cong \prod_{i=1}^{k} \operatorname{End}_{E_{0}}\left(N_{\gamma_{i}}\right) \text {. }
$$

Moreover,

$$
\operatorname{dim}_{E}(N)=\sum_{i=1}^{k} \operatorname{dim}_{E_{0}}\left(N_{\gamma_{i}}\right) .
$$

Proof. Eq. (2.2) readily follows from the description of the grading on $B$, and (2.4) from (2.1), since $N_{\Gamma_{i}}=N_{\gamma_{i}} \otimes_{E_{0}} E$. To prove (2.3), observe that every element in $B_{0}$ maps each $N_{\Gamma_{i}}$ to itself, so

$$
B_{0}=\prod_{i=1}^{k} \operatorname{End}_{E}\left(N_{\Gamma_{i}}\right)_{0} .
$$

Since $N_{\Gamma_{i}}=N_{\gamma_{i}} \otimes_{E_{0}} E$, restriction to $N_{\gamma_{i}}$ defines a canonical isomorphism of $K_{0}$-algebras $\operatorname{End}_{E}\left(N_{\Gamma_{i}}\right)_{0} \cong \operatorname{End}_{E_{0}}\left(N_{\gamma_{i}}\right)$. The proof is thus complete.

This proposition shows that $B_{0}$ is in general semisimple but not simple; however all its simple components are equivalent to $E_{0}$ in the Brauer group $\operatorname{Br}\left(Z\left(E_{0}\right)\right)$. Also, the grade set $\Gamma_{B}$ is in general not a group. We next show how $\Gamma_{E}$ can be detected within $\Gamma_{B}$.

Let $\mathcal{H}_{B}$ be the multiplicative group of homogeneous units of $B$ and let $\Delta_{B} \subseteq$ $\Gamma_{B}$ be the image of $\mathcal{H}_{B}$ under the grade homomorphism mapping each nonempty $\mathcal{H}_{B} \cap B_{\gamma}$ to $\gamma$. The group action of $\mathcal{H}_{B}$ by conjugation on $B$ preserves the grading, so sends $B_{0}$, hence also $Z\left(B_{0}\right)$, to itself. If $b, c \in \mathcal{H}_{B} \cap B_{\gamma}$, then $b^{-1} c \in B_{0}^{\times}$ centralizes $Z\left(B_{0}\right)$; hence $b$ and $c$ have the same action on $Z\left(B_{0}\right)$. Therefore, there is a well-defined homomorphism

$$
\theta_{B}: \Delta_{B} / \Gamma_{K} \rightarrow \operatorname{Aut}\left(Z\left(B_{0}\right) / K_{0}\right),
$$

which maps $\gamma+\Gamma_{K}$ to $z \mapsto b z b^{-1}$ for $z \in Z\left(B_{0}\right)$ and $b \in \mathcal{H}_{B} \cap B_{\gamma}$. Of course, if $B=$ $E$, then $\Delta_{B}=\Gamma_{E}$. The homomorphism $\theta_{B}$ then coincides with the homomorphism $\theta_{E}$ defined in $\left[\mathrm{HW}_{2},(2.2)\right]$. 
Proposition 2.2. Let e be any primitive idempotent of $Z\left(B_{0}\right)$. Then

$$
\Gamma_{E}=\left\{\gamma \in \Delta_{B} \mid \theta_{B}\left(\gamma+\Gamma_{K}\right)(e)=e\right\} .
$$

Moreover, the following diagram is commutative:

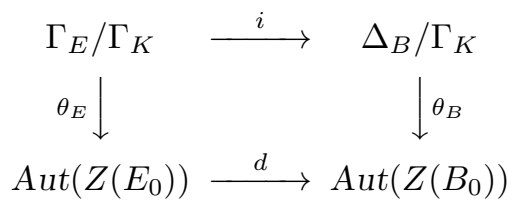

where $i$ is induced by the inclusion $\Gamma_{E} \subseteq \Delta_{B}$ and $d$ is the diagonal map, letting an automorphism of $Z\left(E_{0}\right)$ act on each component of $Z\left(B_{0}\right) \cong Z\left(E_{0}\right) \times \ldots \times Z\left(E_{0}\right)$.

Proof. From the description of $B_{0}$ in Prop. 2.1 it follows that the primitive idempotents of $Z\left(B_{0}\right)$ are the maps $e_{1}, \ldots, e_{k}$ such that $\left.e_{i}\right|_{N_{\Gamma_{i}}}=i d$ and $\left.e_{i}\right|_{N_{\Gamma_{j}}}=0$ for $i \neq j$. Suppose $\gamma \in \Delta_{B}$ is such that $\theta_{B}\left(\gamma+\Gamma_{K}\right)$ fixes some $e_{i}$, and let $h \in \mathcal{H}_{B} \cap B_{\gamma}$. Then $h e_{i}=e_{i} h$; hence $h\left(N_{\Gamma_{i}}\right)=N_{\Gamma_{i}}$ and therefore $\gamma \in \Gamma_{E}$.

Conversely, suppose $\gamma \in \Gamma_{E}$. Take any nonzero $c \in E_{\gamma}$ and any homogeneous $E$-vector space base of $N$ built from bases of the $N_{\Gamma_{i}}$; let $f \in B$ be defined by mapping each base vector $n$ to $n c$. Then $f e_{i}=e_{i} f$ for all $i$ and $f \in \mathcal{H}_{B} \cap B_{\gamma}$, so $\theta_{B}\left(\gamma+\Gamma_{K}\right)$ fixes each $e_{1}, \ldots, e_{k}$. Moreover, $\theta_{B}\left(\gamma+\Gamma_{K}\right)$ induces on each component $e_{i} Z\left(B_{0}\right) \cong Z\left(E_{0}\right)$ of $Z\left(B_{0}\right)$ the automorphism of conjugation by $c$, which is $\theta_{E}\left(\gamma+\Gamma_{K}\right)$. Therefore, diagram (2.6) commutes.

Corollary 2.3. If $B_{0}$ is simple, then $\Gamma_{B}=\Delta_{B}=\Gamma_{E}$ and $\theta_{B}=\theta_{E}$. Moreover, $B$ and $B_{0}$ have the same matrix size, and $[B: K]=\left[B_{0}: K_{0}\right]\left|\Gamma_{B}: \Gamma_{K}\right|$.

Proof. If $B_{0}$ is simple, Prop. 2.1 yields $\Gamma_{B}=\Gamma_{E}$. It also yields $B_{0} \cong \operatorname{End}_{E_{0}}\left(N_{\gamma_{1}}\right)$, where $\operatorname{dim}_{E_{0}}\left(N_{\gamma_{1}}\right)=\operatorname{dim}_{E}(N)$; hence $B$ and $B_{0}$ have the same matrix size. The equalities for $\Gamma_{B}$ and $\theta_{B}$ follow from Prop. 2.2. Since $B \cong_{g} \operatorname{End}_{E}(N)$, we have

$$
\begin{aligned}
{[B: K] } & =\left(\operatorname{dim}_{E}(N)\right)^{2}[E: K]=\left(\operatorname{dim}_{E_{0}}\left(N_{\gamma_{1}}\right)\right)^{2}\left[E_{0}: K_{0}\right]\left|\Gamma_{E}: \Gamma_{K}\right| \\
& =\left[B_{0}: K_{0}\right]\left|\Gamma_{B}: \Gamma_{K}\right| .
\end{aligned}
$$

Example 2.4. With the notation of Prop. 2.1, if the dimensions $\operatorname{dim}_{E_{0}}\left(N_{\gamma_{1}}\right), \ldots$, $\operatorname{dim}_{E_{0}}\left(N_{\gamma_{k}}\right)$ are all different, then every invertible homogeneous element in $B$ has grade in $\Gamma_{E}$; therefore, $\Delta_{B}=\Gamma_{E}$ and $\theta_{B}=\theta_{E}$.

We can now see how the gauges considered here are related to Morandi value functions. The main earlier approach to value functions for central simple algebras is that of P. Morandi in $\left[\mathrm{M}_{2}\right.$ and $[\mathrm{MW}$. Let $A$ be a central simple algebra over a field $F$ with a valuation $v$. Let $y: A \rightarrow \Gamma \cup\{\infty\}$ be a surmultiplicative $v$-value function, and let

$$
\operatorname{st}(y)=\left\{a \in A^{\times} \mid y\left(a^{-1}\right)=-y(a)\right\},
$$

a subgroup of $A^{\times}$; cf. Lemma 1.3. Let $V_{y}=A^{\geq 0}$, the "valuation ring" of $y$, and let $A_{0}=A^{\geq 0} / A^{>0}$, the degree 0 part of $\operatorname{gr}_{y}(A)$. Then, $y$ is a Morandi value function if

(i) $A_{0}$ is a simple ring;

(ii) $y(\operatorname{st}(y))=\Gamma_{A}$. 
When this occurs, it is known that $V_{y}$ is a Dubrovin valuation ring integral over its center, which is $V_{v}$, and $y$ is completely determined by $V_{y}$. Conversely, to every Dubrovin valuation ring $B$ of $A$ with $B$ integral over its center, there is a canonically associated Morandi value function $y_{B}$ with $V_{y_{B}}=B$. (For the theory of Dubrovin valuation rings, see $\mathrm{MMU}, \mathrm{W}_{2}$, or $[\mathrm{G}$, and the references given there. In particular, it is known that for every central simple algebra $A$ over a field $F$ and every valuation $v$ on $F$ there is a Dubrovin valuation $\operatorname{ring} B$ of $A$ with $B \cap F=V_{v}$, and such a $B$ is unique up to conjugacy, so unique up to isomorphism. See $\mathrm{W}_{2}$, Th. F] for characterizations of when $B$ is integral over $V_{v}$.) For a Morandi value function $y$ on $A$, the defect $\delta(y)$ (an integer) is defined by

$$
\delta(y)=[A: F] /\left[A_{0}: F_{0}\right]\left|\Gamma_{A}: \Gamma_{F}\right| .
$$

If $\left(F^{h}, v^{h}\right)$ is a Henselization of $(F, v)$, and $D^{h}$ is the division algebra Brauerequivalent to $A \otimes_{F} F^{h}$, it is known (see $\left[\mathrm{W}_{2}\right.$, Th. C]) that $\delta(y)$ coincides with the defect of the valuation on $D^{h}$ extending $v^{h}$ on $F^{h}$. Hence, $\delta(y)=1$ if $\operatorname{char}(\bar{F})=0$ and $\delta(y)$ is a power of $\operatorname{char}(\bar{F})$ otherwise. The requirement of integrality of $V_{y}$ over $V_{v}$ has been a significant limitation in applying the machinery of Dubrovin valuation rings in connection with value functions on central simple algebras.

Proposition 2.5. Let $A$ be a central simple algebra over a field $F$ with a valuation $v$, and let $y$ be a surmultiplicative $v$-value function on $A$. Then, $y$ is a Morandi value function on $A$ with $\delta(y)=1$ if and only if $y$ is a gauge on $A$ with $A_{0}$ simple.

Proof. $\Rightarrow$ Suppose $y$ is a Morandi value function on $A$ with $\delta(y)=1$. For any $\gamma \in \Gamma_{A}=y(\operatorname{st}(y))$, there is $u_{\gamma} \in \operatorname{st}(y)$ with $y\left(u_{\gamma}\right)=\gamma$. Then, $u_{\gamma}^{\prime}$ is a homogeneous unit of $\operatorname{gr}(A)$, by Lemma 1.3. Take any nonzero homogeneous ideal $I$ of $\operatorname{gr}(A)$. Then, there is a $\gamma \in \Gamma_{A}$ with $I \cap A_{\gamma} \neq\{0\}$. So, for the ideal $I \cap A_{0}$ of $A_{0}$ we have $I \cap A_{0} \supseteq u_{\gamma}^{\prime-1}\left(I \cap A_{\gamma}\right) \neq\{0\}$. Because $A_{0}$ is a simple ring, we must have $I \cap A_{0}=A_{0}$, so $1 \in I$, so $I=\operatorname{gr}(A)$. Thus, $\operatorname{gr}(A)$ is a simple ring, which is finite-dimensional over $\operatorname{gr}(F)$ by Prop. 1.1(ii). Furthermore, as $\delta(y)=1$, Cor. 2.3 yields

$$
[A: F]=\left[A_{0}: F_{0}\right]\left|\Gamma_{A}: \Gamma_{F}\right|=[\operatorname{gr}(A): \operatorname{gr}(F)] .
$$

Hence, $y$ is a gauge on $A$. By hypothesis, $A_{0}$ is simple.

$\Leftarrow$ Suppose $y$ is a gauge on $A$ with $A_{0}$ simple. Since $\operatorname{gr}(A)$ is graded semisimple, it is a graded direct product of graded simple rings. But, if $\operatorname{gr}(A)$ has nontrivial graded direct product decomposition $\operatorname{gr}(A)=C \times D$, then $A_{0}=C_{0} \times D_{0}$ with $C_{0}$ and $D_{0}$ nontrivial. This cannot occur as $A_{0}$ is simple. Hence, $\operatorname{gr}(A)$ is graded simple. (This also follows from the simplicity of $A$. See Cor. 3.7 below.) Since $A_{0}$ is simple, Cor. 2.3 applies with $B=\operatorname{gr}(A)$. The equality $\Gamma_{\operatorname{gr}(A)}=\Delta_{\operatorname{gr}(A)}$ from Cor. 2.3 shows that for each $\gamma \in \Gamma_{A}=\Gamma_{\operatorname{gr}(A)}$, there is a homogeneous unit $u$ in $A_{\gamma}$. Pick any $a \in A$ with $y(a)=\gamma$ and $a^{\prime}=u$. Then, $a \in$ st $(y)$ by Lemma 1.3. Hence, $y(\operatorname{st}(y))=\Gamma_{A}$, proving that $y$ is a Morandi value function. Furthermore, as $y$ is a norm on $A$, by Cor. 2.3 and Prop. 1.1(ii) we have

$$
\delta(y)=[A: F] /\left[A_{0}: F_{0}\right]\left|\Gamma_{A}: \Gamma_{F}\right|=[A: F] /[\operatorname{gr}(A): \operatorname{gr}(F)]=1 .
$$

\section{Gauges over Henselian fields}

The next theorem provides the key link between a gauge on a simple algebra over a Henselian field and the valuation on the associated division algebra. We write $m s(B)$ for the matrix size of a simple (or graded simple) algebra $B$. 
Theorem 3.1. Let $F$ be a field with a Henselian valuation $v$. Let $A$ be a simple $F$-algebra with an $F$-gauge $y$. Let $D$ be the division algebra Brauer-equivalent to $A$, and let $w$ be the valuation on $D$ extending $v$ on $F$. Then, $\operatorname{gr}(A)$ is simple and $w$ is defectless. Furthermore, there is a finite-dimensional right $D$-vector space $M$ with a w-norm $\alpha$ such that

$$
A \cong \operatorname{End}_{D}(M), \quad \operatorname{gr}(A) \cong \operatorname{End}_{\operatorname{gr}(D)}(\operatorname{gr}(M)),
$$

and $y$ on $A$ corresponds to the $F$-gauge $y_{\alpha}$ on $\operatorname{End}_{D}(M)$ induced by $\alpha$ as in 1.3 . In particular, $\operatorname{ms}(A)=\operatorname{ms}(\operatorname{gr}(A))$, and the gauge $y$ is tame if and only if $w$ is a tame F-gauge.

Proof. We first show that the gauge ring $V_{A}=\{a \in A \mid y(a) \geq 0\}$ is integral over $V_{F}$. Let $C=\operatorname{End}_{F}(A)$, and let $z=z_{y}: C \rightarrow \Gamma \cup\{\infty\}$ be the gauge on $C$ arising from the norm $y$ on $A$, as in $\$ 1.3$. Take any $a \in V_{A}$. For each $b \in A$, we have

$$
y(a b)-y(b) \geq y(a)+y(b)-y(b) \geq 0 .
$$

This shows that for the left multiplication map $\lambda_{a} \in C$ given by $b \mapsto a b$, we have $z\left(\lambda_{a}\right) \geq 0$. That is, $\lambda_{a} \in V_{C}$. Lemma 1.20 shows that $V_{C}$ is integral over $V_{F}$; hence $\lambda_{a}$ is integral over $V_{F}$, and so therefore is $a$. This shows that $V_{A}$ is integral over $V_{F}$.

Because $V_{F}$ is Henselian and $V_{A}$ is integral over $V_{F}$, we can lift idempotents from any homomorphic image of $V_{A}$ to $V_{A}$, by [MMU, Th. A.18, p. 180]. (This is also deducible from results in Nagata's book [N]: if $J$ is any ideal of $V_{A}$, take any $b \in V_{A}$ with $b^{2}-b \in J$, and let $T=V_{F}[b]$, which is a commutative ring finitely generated as a $V_{F}$-module, as $b$ is integral over $V_{F}$. By [N] Th. 43.15, p. 185], $T$ is a direct product $T=S_{1} \times \ldots \times S_{k}$ where each $S_{i}$ is a local ring. Let $J \cap T=K_{1} \times \ldots \times K_{k}$, so $T /(J \cap T) \simeq S_{1} / K_{1} \times \ldots \times S_{k} / K_{k}$. Since each $S_{i} / K_{i}$ is local, it has no nontrivial idempotent. Therefore, by working componentwise, we see that there is an idempotent $e$ of $T$ with $e \equiv b \bmod J \cap T$.) We use this idempotent-lifting property first to show that $\operatorname{gr}(A)$ is graded simple. Since $\operatorname{gr}(A)$ is graded semisimple, if it were not graded simple, it would have a nontrivial central homogeneous idempotent $\widetilde{c}$. Since $\widetilde{c}^{2}=\widetilde{c}$, necessarily $\widetilde{c} \in \operatorname{gr}(A)_{0}=V_{A} / M_{A}$. Therefore, the idempotent lifting result says that there is an idempotent $c \in V_{A}$ with $c^{\prime}=\widetilde{c}$ in $\operatorname{gr}(A)_{0}$. So, $y(c)=0$. For the complementary idempotent $1-c$, we have $(1-c)^{\prime}=1^{\prime}-c^{\prime}$, since $y(c)=y(1)$ and $c^{\prime} \neq 1^{\prime}$. Now, by two applications of Lemma 1.7

$$
\operatorname{gr}(c A(1-c))=c^{\prime} \operatorname{gr}(A(1-c))=\widetilde{c} \operatorname{gr}(A)\left(1^{\prime}-\widetilde{c}\right)=\{0\},
$$

as $\widetilde{c}$ is a central idempotent of $\operatorname{gr}(A)$. Hence, $c A(1-c)=\{0\}$, and likewise $(1-c) A c=\{0\}$. Therefore, $c \in Z(A)$, so $A \cong c A c \times(1-c) A(1-c)$, contradicting the graded simplicity of $A$. Thus, $\operatorname{gr}(A)$ must be graded simple.

Now, let $\widetilde{e}$ be any primitive homogeneous idempotent of the graded simple ring $\operatorname{gr}(A)$. Just as for $\widetilde{c}$ above, we have $\widetilde{e} \in \operatorname{gr}(A)_{0}$ and there is an idempotent $e \in V_{A}$ with $e^{\prime}=\widetilde{e}$ in $\operatorname{gr}(A)$. So, $y(e)=0$. Now, $\left.y\right|_{e A e}$ is a surmultiplicative $F$-value function (when we identify $F$ with $e F$ ). By Lemma 1.7 $\operatorname{gr}(e A e)=e^{\prime} \operatorname{gr}(A) e^{\prime}$, which is a graded division ring as $e^{\prime}$ is primitive. Because $\operatorname{gr}(e A e)$ has no zero divisors, (1.5) shows that

$$
y(b d)=y(b)+y(d) \quad \text { for all } b, d \in e A e .
$$


Therefore, the finite-dimensional $F$-algebra $e A e$ has no zero divisors and is thus a division ring. Hence, $e$ is a primitive idempotent of $A$, and $e A e \cong D$. We identify $e A e$ with $D$ by any such $F$-isomorphism. Formula (3.1) further shows that $\left.y\right|_{D}$ is a valuation restricting to $v$ on $F$. Hence, $\left.y\right|_{D}=w$, as $v$ has a unique extension to $D$. Since the gauge $y$ is an $F$-norm on $A$, its restriction to $D$ is also an $F$-norm by Prop. 1.1(iii). So, $D$ is defectless over $F$.

Set $M=A e$, which is a right $D=e A e$-vector space. Let $\alpha=\left.y\right|_{M}$, which is an $F$-norm by Prop. 1.1(iii). By Lemma 1.7 $\operatorname{gr}(M)=\operatorname{gr}(A) \widetilde{e}$, which is a (unital) right $\widetilde{e} g r(A) \widetilde{e}=\operatorname{gr}(D)$-module. Hence, as $m^{\prime} d^{\prime} \neq 0$ in $\operatorname{gr}(M)$ whenever $m^{\prime} \neq 0$ and $d^{\prime} \neq 0$, formula (1.5) yields

$$
\alpha(m d)=y(m d)=y(m)+y(d)=\alpha(m)+w(d) \quad \text { for all } m \in M, d \in D .
$$

This shows that $\alpha$ is actually a $D$-value function on $M$. Furthermore,

$$
\begin{aligned}
\operatorname{dim}_{\operatorname{gr}(D)}(\operatorname{gr}(M)) & =\operatorname{dim}_{\operatorname{gr}(F)}(\operatorname{gr}(M)) /[\operatorname{gr}(D): \operatorname{gr}(F)] \\
& =\operatorname{dim}_{F}(M) /[D: F]=\operatorname{dim}_{D}(M),
\end{aligned}
$$

which shows that $\alpha$ is a $D$-norm on $M$.

By Wedderburn's Theorem, the map

$$
\beta: A \rightarrow \operatorname{End}_{D}(M) \text { given by } \beta(a)(b)=a b
$$

is an $F$-algebra isomorphism. We prove that $\beta$ induces an isomorphism of graded rings. Let $y_{\alpha}: \operatorname{End}_{D}(M) \rightarrow \Gamma \cup\{\infty\}$ be the $F$-gauge on $\operatorname{End}_{D}(M)$ induced by the $D$-norm $\alpha$ on $M$, as in $\$ 1.3$. That is, for $f \in \operatorname{End}_{D}(M)$, we have $y_{\alpha}(f)=$ $\min _{m \in M, m \neq 0}(\alpha(f(m))-\alpha(m))$. We claim that for every nonzero $a \in A$,

$$
y_{\alpha}(\beta(a))=y(a) .
$$

For, we have $y_{\alpha}(\beta(a))=\min _{m \in M, m \neq 0}(y(a m)-y(m)) \geq y(a)$, since $y(a m) \geq y(a)+$ $y(m)$ for all $m$. Suppose $y_{\alpha}(\beta(a))>y(a)$. Then, $y(a m)-y(m)>y(a)$, for all $m$. Hence, $a^{\prime} \cdot m^{\prime}=0$ in $\operatorname{gr}(M)$ for all $m \in M$, showing that $a^{\prime} \operatorname{gr}(M)=0$, since $\operatorname{gr}(M)$ is generated by its homogeneous elements. But, $\operatorname{gr}(M)=\operatorname{gr}(A) e^{\prime}$. Thus, $0=a^{\prime} \operatorname{gr}(M)=a^{\prime} \operatorname{gr}(A) e^{\prime}$. Because $\operatorname{gr}(A)$ is graded simple, we have $\operatorname{gr}(A) e^{\prime} \operatorname{gr}(A)=$ $\operatorname{gr}(A)$, and hence $0=a^{\prime} \operatorname{gr}(A) e^{\prime} \operatorname{gr}(A)=a^{\prime} \operatorname{gr}(A)$, which shows that $a^{\prime}=0$. This contradicts $a \neq 0$, proving (3.2). From (3.2) and Prop. 1.19 we have

$$
\operatorname{gr}_{y}(A) \cong_{g} \operatorname{gr}_{y_{\alpha}}\left(\operatorname{End}_{D}(M)\right) \cong_{g} \operatorname{End}_{g r_{w}(D)}\left(\operatorname{gr}_{\alpha}(M)\right),
$$

as desired. Note that $m s(A)=\operatorname{dim}_{D}(M)=\operatorname{dim}_{\operatorname{gr}(D)}(\operatorname{gr}(M))=m s(\operatorname{gr}(A))$. Moreover, Prop. 1.19 shows that the gauge $y_{\alpha}$ is tame if and only if $w$ is a tame $F$-gauge. This completes the proof of Theorem 3.1

Corollary 3.2. Let $F$ be a field with a Henselian valuation $v$, let $D$ be a (finitedimensional) division algebra over $F$, and let $w$ be the valuation on $D$ extending $v$. Then, $w$ is the only possible v-gauge on $D ; w$ is a v-gauge if and only if $D$ is defectless over $F$.

Proof. Let $y$ be a $v$-gauge on $D$. Theorem 3.1 shows that $g r_{y}(D) \cong_{g} g r_{w}(D)$, which is a graded division ring. Therefore, as we saw in the proof of Theorem 3.1, $y$ is a valuation on $D$, extending $v$ on $F$. But $w$ is the unique extension of $v$ to $D$, so $y=w$. By Prop. 1.12 $w$ is a $v$-gauge if and only if $D$ is defectless over $F$. 
Because of the compatibility of gauges with direct products, we can easily extend Theorem 3.1 to semisimple algebras over a Henselian field.

Theorem 3.3. Let $F$ be a field with a Henselian valuation $v$, and let $A$ be $a$ semisimple $F$-algebra with an F-gauge $y$. Let $A_{1}, \ldots, A_{n}$ be the simple components of $A$,

$$
A=A_{1} \times \ldots \times A_{n} .
$$

For $i=1, \ldots, n$, the restriction $\left.y\right|_{A_{i}}$ is a gauge on $A_{i}$, the graded algebra $\operatorname{gr}_{\left.y\right|_{A_{i}}}\left(A_{i}\right)$ is graded simple with $\operatorname{ms}\left(A_{i}\right)=\operatorname{ms}\left(\operatorname{gr}\left(A_{i}\right)\right)$, and $\operatorname{gr}\left(A_{1}\right), \ldots, \operatorname{gr}\left(A_{n}\right)$ are the graded simple components of $\operatorname{gr}(A)$,

$$
\operatorname{gr}(A)=\operatorname{gr}\left(A_{1}\right) \times \ldots \times \operatorname{gr}\left(A_{n}\right) .
$$

For $a=\left(a_{1}, \ldots, a_{n}\right) \in A_{1} \times \ldots \times A_{n}$,

$$
y(a)=\min _{1 \leq i \leq n}\left(\left.y\right|_{A_{i}}\left(a_{i}\right)\right) .
$$

Moreover, the gauge $y$ is tame if and only if each $\left.y\right|_{A_{i}}$ is tame.

Proof. This is immediate from Theorem 3.1 and Prop. 1.6

If the valuation $v$ is not Henselian, we may still apply Theorem 3.3 after scalar extension to a Henselization $\left(F^{h}, v^{h}\right)$ of $(F, v)$. If $y$ is a $v$-gauge on the semisimple $F$-algebra $A$, then Cor. 1.26 shows that $y \otimes v^{h}$ is a $v^{h}$-gauge on $A \otimes_{F} F^{h}$ with $\operatorname{gr}\left(A \otimes_{F} F^{h}\right)=\operatorname{gr}(A)$, since $\operatorname{gr}\left(F^{h}\right)=\operatorname{gr}(F)$. Let $B_{1}, \ldots, B_{n}$ be the simple components of $A \otimes_{F} F^{h}$,

$$
A \otimes_{F} F^{h}=B_{1} \times \ldots \times B_{n} .
$$

For $i=1, \ldots, n$, let $D_{i}$ be the division algebra Brauer-equivalent to $B_{i}$.

Proposition 3.4. With the notation above, let $\ell$ be the number of simple components of $A$ and $\ell^{\prime}$ be the number of graded simple components of $\operatorname{gr}(A)$. Then $\ell \leq \ell^{\prime}=n$. Moreover, the following conditions are equivalent:

(i) $y$ is a tame $v$-gauge;

(ii) $y \otimes v^{h}$ is a tame $v^{h}$-gauge;

(iii) for $i=1, \ldots, n$, the unique valuation $w_{i}$ on $D_{i}$ extending $v^{h}$ is a tame $v^{h}$-gauge.

Proof. The equality $\ell^{\prime}=n$ follows from Theorem 3.3 and the equality $\ell \leq n$ is clear. The equivalence (i) $\Longleftrightarrow$ (ii) readily follows from Cor. 1.26. For $i=1, \ldots, n$, let $y_{i}$ be the restriction of $y \otimes v^{h}$ to $B_{i}$. Theorem 3.3 shows that $y \otimes v^{h}$ is a tame $v^{h}$-gauge if and only if each $y_{i}$ is a tame $v^{h}$-gauge. By Theorem 3.1 , this condition is equivalent to (iii).

Corollary 3.5. Suppose $A$ is a simple algebra finite-dimensional over a field $F$ with valuation $v$. If $y$ is a $v$-gauge on $A$, then the number of simple components of $\operatorname{gr}(A)$ equals the number of extensions of $v$ from $F$ to $Z(A)$.

Proof. Let $\left(F^{h}, v^{h}\right)$ be a Henselization of $(F, v)$, and let $K=Z(A)$. Since $F^{h}$ is separable over $F$, we can write $K \otimes_{F} F^{h}=L_{1} \times \ldots \times L_{n}$, where each $L_{i}$ is a field. Then, $A \otimes_{F} F^{h} \cong A \otimes_{K}\left(K \otimes_{F} F^{h}\right) \cong B_{1} \times \ldots \times B_{n}$, where each $B_{i}=A \otimes_{K} L_{i}$, a central simple $L_{i}$-algebra. With respect to the $F^{h}$-gauge $y \otimes v^{h}$ on $A \otimes_{F} F^{h}$, Theorem 3.3 shows that

$$
\operatorname{gr}\left(A \otimes_{F} F^{h}\right) \cong g \operatorname{gr}\left(B_{1}\right) \times \ldots \times \operatorname{gr}\left(B_{n}\right)
$$


with each $\operatorname{gr}\left(B_{i}\right)$ simple. Now, $\left.y\right|_{K}$ is a surmultiplicative norm on $K$ by Prop.1.1(iii), and $\operatorname{gr}(K)$ is semisimple since it is a central subalgebra of the semisimple $\operatorname{gr}(F)$-algebra $\operatorname{gr}(A)$; so, $\left.y\right|_{K}$ is a gauge. Hence, by Prop. 1.8 and Cor. [1.9, the number of simple summands of $\operatorname{gr}(K)$ equals the number of extensions of $v$ to $K$. We have $\left.y\right|_{K} \otimes v^{h}$ is a gauge on $K \otimes_{F} F^{h}$, and

$$
\operatorname{gr}\left(K \otimes_{F} F^{h}\right) \cong g \operatorname{gr}\left(L_{1}\right) \times \ldots \times \operatorname{gr}\left(L_{n}\right)
$$

by Prop.11.11, Furthermore, each $\operatorname{gr}\left(L_{i}\right)$ is simple by Cor. 1.9 and Prop. 1.8 because the Henselian valuation $v^{h}$ has a unique extension from $F^{h}$ to $L_{i}$. Since $\operatorname{gr}(A) \cong_{g}$ $\operatorname{gr}\left(A \otimes_{F} F^{h}\right)$ and $\operatorname{gr}(K) \cong_{g} \operatorname{gr}\left(K \otimes_{F} F^{h}\right)$ by Cor. 1.26, equations (3.3) and (3.4) show that

$$
\begin{aligned}
n & =\text { number of simple components of } \operatorname{gr}(A) \\
& =\text { number of simple components of } \operatorname{gr}(K) \\
& =\text { number of extensions of } v \text { to } K .
\end{aligned}
$$

Corollary 3.6. If $\operatorname{char}(\bar{F})=0$, then every $F$-gauge on a semisimple $F$-algebra is tame.

Proof. Condition (iii) of Prop. 3.4 holds if $\operatorname{char}(\bar{F})=0$, by Prop. 1.13 ,

In the rest of this section, we consider the case where the semisimple $F$-algebra $A$ is central simple. Recall from $\mathrm{HW}_{2}$ that the graded Brauer group $G B r(E)$ of a graded field $E$ can be defined on the same model as the classical Brauer group of fields. The elements of $\operatorname{GBr}(E)$ are graded isomorphism classes of graded division algebras with center $E$.

Corollary 3.7. Let $(F, v)$ be a valued field and let $\left(F^{h}, v^{h}\right)$ be a Henselization of $(F, v)$. Let $A$ be a central simple $F$-algebra, let $D$ be the division algebra Brauerequivalent to $A \otimes_{F} F^{h}$, and let $w$ be the valuation on $D$ extending $v^{h}$. Let $y$ be any v-gauge on $A$. Then, the $\operatorname{gr}_{v}(F)$-algebra $\operatorname{gr}_{y}(A)$ is graded simple and Brauerequivalent to $\operatorname{gr}_{w}(D)$. Moreover, if $y$ is tame, then $D$ is tame (see Def. 1.14) and $\mathrm{gr}_{y}(A)$ is central simple over $\mathrm{gr}_{v}(F)$.

Proof. Let $A^{h}=A \otimes_{F} F^{h}$ and let $y^{h}=y \otimes v^{h}$. Theorem 3.3 shows that $g r_{y^{h}}\left(A^{h}\right)$ is graded simple, and Theorem 3.1 yields $\operatorname{gr}_{y^{h}}\left(A^{h}\right) \cong{ }_{g} \operatorname{End}_{\operatorname{gr}(D)}(\operatorname{gr}(M))$ for some finite-dimensional right $D$-vector space $M$; hence $\operatorname{gr}_{y^{h}}\left(A^{h}\right)$ is Brauer-equivalent to $\operatorname{gr}(D)$. These properties carry over to $\operatorname{gr}_{y}(A)$ because $\operatorname{gr}_{y}(A)=\operatorname{gr}_{y^{h}}\left(A^{h}\right)$ by Cor. 1.26, since $\operatorname{gr}\left(F^{h}\right)=\operatorname{gr}(F)$. When $y$ is tame, it follows by definition that $Z(\operatorname{gr}(A))=\operatorname{gr}(F)$, and from Prop. 3.4 that $D$ is tame.

For any valued field $(F, v)$, define the tame part of the Brauer group $\operatorname{Br}(F)$ (with respect to $v$ ) to be

$\operatorname{TBr}(F)=\{[A] \mid A$ is a central simple $F$-algebra with a tame $F$-gauge $\}$.

Cor. 3.7 shows that if $y$ is a tame $v$-gauge on a central simple $F$-algebra $A$, then the Brauer class $\left[g_{y}(A)\right]$ in $\operatorname{GBr}(\operatorname{gr}(F))$ does not depend on the choice of the tame $v$-gauge but only on the Brauer class of $A$, since it coincides with the Brauer class $[\operatorname{gr}(D)]$, where $D$ is the division algebra Brauer-equivalent to $A \otimes_{F} F^{h}$. Therefore, there is a well-defined map

$$
\Psi: \operatorname{TBr}(F) \rightarrow \operatorname{GBr}(\operatorname{gr}(F)), \quad[A] \mapsto\left[\operatorname{gr}_{y}(A)\right] \text { for any } v \text {-gauge } y \text { on } A .
$$


By Cor. 1.28, $\operatorname{TBr}(F)$ is a subgroup of $\operatorname{Br}(F)$ and the map $\Psi$ is a group homomorphism.

Theorem 3.8. The kernel of $\Psi$ consists of the elements in $\operatorname{TBr}(F)$ which are split by any Henselization $F^{h}$ of $F$ with respect to $v$. Moreover, for any valued field $(L, w)$ extending $(F, v)$, there is a commutative diagram

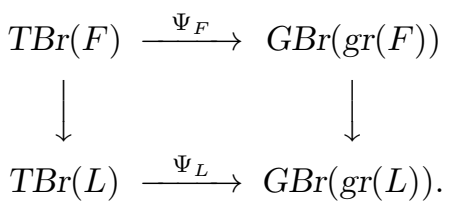

If $v$ is Henselian, then $\Psi$ is an index-preserving group isomorphism.

Proof. Cor. 1.26 shows that the scalar extension map $-\otimes_{F} L$ sends $\operatorname{TBr}(F)$ to $\operatorname{TBr}(L)$, and that diagram (3.5) is commutative. When $v$ is Henselian, Theorem 3.1 shows that $m s\left(\operatorname{gr}_{y}(A)\right)=m s(A)$ for any central simple $F$-algebra; hence $\Psi$ is indexpreserving and injective. In this Henselian case, $\Psi$ is also surjective, by $\mathrm{HW}_{2}$, Theorem 5.3]. Another more direct proof of the surjectivity is possible, by showing that we can construct algebra classes of unramified algebras and inertially split cyclic algebras and tame totally ramified symbol algebras over $F$ which map onto generators of $\operatorname{GBr}(F)$. No longer assuming that $v$ is Henselian, take $L=F^{h}$ in the commutative diagram (3.5); since $\Psi_{F^{h}}$ is bijective and $\operatorname{gr}\left(F^{h}\right)=\operatorname{gr}(F)$, the kernel of $\Psi_{F}$ is the kernel of the scalar extension map $\operatorname{TBr}(F) \rightarrow \operatorname{TBr}\left(F^{h}\right)$.

This theorem generalizes $\left[\mathrm{HW}_{2}\right.$, Th. 5.3], which showed that $\Psi_{F}$ is a group isomorphism when $v$ is Henselian. The proof given here is vastly simpler than the one in $\mathrm{HW}_{2}$.

\section{Applications}

The utility of Theorems 3.3 and 3.1 depends on being able to construct gauges on algebras over valued fields. We give several examples where this can be done, obtaining as a result considerably simplified and more natural proofs of some earlier theorems. In each case, we use the following result, which can be viewed as a detection theorem: it allows one to use a gauge to determine whether the division algebra $D$ Brauer-equivalent to a given central simple algebra $A$ has a valuation extending a given valuation on the center, without first determining $D$.

Theorem 4.1. Let $(F, v)$ be a valued field, let $A$ be a central simple $F$-algebra, and let $D$ be the division algebra Brauer-equivalent to $A$. Suppose $A$ has an F-gauge $y$. Then, $\operatorname{gr}(A)$ is simple, and $\operatorname{ms}(\operatorname{gr}(A)) \geq \operatorname{ms}(A)$. Moreover, the following conditions are equivalent:

(i) $v$ extends to a valuation on $D$;

(ii) $\operatorname{ms}(\operatorname{gr}(A))=\operatorname{ms}(A)$;

(iii) $[D: F]=[E: \operatorname{gr}(F)]$, where $E$ is the graded division algebra Brauer-equivalent to $\operatorname{gr}(A)$.

When these conditions hold, $\operatorname{gr}(D) \cong_{g} E$ and $\operatorname{gr}(A) \cong_{g} \operatorname{End}_{\operatorname{gr}(D)}(N)$ for some graded right $\operatorname{gr}(D)$-vector space $N$. Hence, $\bar{D}$ is Brauer equivalent to any simple component of $A_{0}$, and $\Gamma_{D}$ and $\theta_{D}$ are determinable from $\operatorname{gr}(A)$, as described in Prop. 2.2. 
Proof. Let $\left(F^{h}, v^{h}\right)$ be a Henselization of $(F, v)$. Let $A^{h}=A \otimes_{F} F^{h}$ and $D^{h}=$ $D \otimes_{F} F^{h}$. So, $A^{h} \cong M_{n}\left(D^{h}\right)$, where $A \cong M_{n}(D)$. As noted in the proof of Cor. 3.7. $y \otimes v^{h}$ is an $F^{h}$-gauge on $A^{h}$ with $\operatorname{gr}\left(A^{h}\right) \cong_{g} \operatorname{gr}(A)$. Since $A^{h}$ is simple, Theorem 3.3 shows that $\operatorname{gr}\left(A^{h}\right)$ (so also $\operatorname{gr}(A)$ ) is graded simple, and that $\operatorname{ms}(\operatorname{gr}(A))=\operatorname{ms}\left(A^{h}\right)$. Of course, $\operatorname{ms}\left(A^{h}\right) \geq m s(A)$. Thus, $\operatorname{ms}(\operatorname{gr}(A))=m s(A)$ iff $m s\left(A^{h}\right)=m s(A)$ iff $D^{h}$ is a division ring iff $v$ extends to a valuation on $D$, by Morandi's theorem $\mathrm{M}_{1}$, Th. 2].

Let $\widetilde{D}$ be the division algebra Brauer-equivalent to $A^{h}$. By Theorem 3.1 we have $A^{h} \cong \operatorname{End}_{\widetilde{D}}(N)$ for some right $\widetilde{D}$-vector space $N$ with a $\widetilde{D}$-norm, and $\operatorname{gr}\left(A^{h}\right) \cong_{g}$ $\operatorname{End}_{\operatorname{gr}(\widetilde{D})}(\operatorname{gr}(M))$. Hence, $E \cong_{g} \operatorname{gr}(\widetilde{D})$, by the uniqueness part of the graded Wedderburn theorem. Since Theorem 3.1 also says that the valuation on $\widetilde{D}$ extending $v^{h}$ is an $F^{h}$-gauge, we have $[E: \operatorname{gr}(F)]=\left[\operatorname{gr}(\widetilde{D}): \operatorname{gr}\left(F^{h}\right)\right]=\left[\widetilde{D}: F^{h}\right]$. But, $[D: F]=\left[D^{h}: F^{h}\right]$. Hence, $[E: \operatorname{gr}(F)]=[D: F]$ iff $\left[\widetilde{D}: F^{h}\right]=\left[D^{h}: F^{h}\right]$ iff $D^{h}$ is a division ring iff (as above) $v$ extends to $D$. When this occurs, since the valuation on $D^{h}$ extends the one on $D$, we have an inclusion $\iota: \operatorname{gr}(D) \hookrightarrow \operatorname{gr}\left(D^{h}\right)$, but $\iota$ is actually an isomorphism, since $\bar{D} \cong \overline{D^{h}}$ and $\Gamma_{D}=\Gamma_{D^{h}}$ by $\left[\mathrm{M}_{1}\right.$, Th. 2]; hence $D_{0} \cong D_{0}^{h}$ and $\Gamma_{\operatorname{gr}(D)}=\Gamma_{\operatorname{gr}\left(D^{h}\right)}$. Thus, $E \cong_{g} \operatorname{gr}(\widetilde{D}) \cong_{g} \operatorname{gr}\left(D^{h}\right) \cong_{g} \operatorname{gr}(D)$, so that $\operatorname{gr}(A) \cong_{g} \operatorname{End}_{\operatorname{gr}(D)}(N)$. Hence the Brauer class of $\bar{D}=D_{0}$ coincides with that of any simple component of $A_{0}$, and $\theta_{D}$ is determinable from $\operatorname{gr}(A)$ as described in Prop. 2.2.

4.1. Crossed products. We now show how to construct tame gauges on crossed product algebras when the Galois extension is indecomposed and defectless with respect to the valuation.

Let $K / F$ be a finite Galois extension of fields, and let $G$ be the Galois group $\mathcal{G}(K / F)$. Let $A$ be a crossed product algebra $(K / F, G, f)$, where $f$ is a 2-cocycle in $Z^{2}\left(G, K^{\times}\right)$, and assume for convenience that $f$ is normalized. Explicitly, write

$$
A=\bigoplus_{\sigma \in G} K x_{\sigma}
$$

where

$x_{\text {id }}=1, x_{\sigma} c x_{\sigma}^{-1}=\sigma(c)$ for all $c \in K$ and $x_{\sigma} x_{\tau}=f(\sigma, \tau) x_{\sigma \tau}$ for all $\sigma, \tau \in G$.

Assume $v$ is a valuation on $F$ which has a unique extension to a valuation $w$ of $K$ and that $w$ is defectless over $v$. Thus, $w$ is a $v$-norm on $K$ as an $F$-vector space, and every automorphism $\sigma \in \mathcal{G}(K / F)$ induces an automorphism $\sigma^{\prime}$ of $\operatorname{gr}(K)$. Assume further that $\bar{K}$ is separable over $\bar{F}$ and that $\operatorname{char}(\bar{F}) \nmid\left|\Gamma_{K}: \Gamma_{F}\right|$. Then $\operatorname{gr}(K)$ is Galois over $\operatorname{gr}(F)$ and the canonical map $\mathcal{G}(K / F) \rightarrow \mathcal{G}(\operatorname{gr}(K) / \operatorname{gr}(F))$ given by $\sigma \mapsto \sigma^{\prime}$ is an isomorphism. Let $G^{\prime}=\mathcal{G}(\operatorname{gr}(K) / \operatorname{gr}(F))$ and define $f^{\prime}: G^{\prime} \rightarrow \operatorname{gr}(K)^{\times}$ by $f^{\prime}\left(\sigma^{\prime}, \tau^{\prime}\right)=f(\sigma, \tau)^{\prime}$; then $f^{\prime} \in Z^{2}\left(G^{\prime}, \operatorname{gr}(K)^{\times}\right)$and we may consider the crossed product algebra $\left(\operatorname{gr}(K) / \operatorname{gr}(F), G^{\prime}, f^{\prime}\right)$. This is a graded simple $\operatorname{gr}(F)$-algebra; see $\mathrm{HW}_{2}$, Lemma 3.1].

Toward defining a $v$-gauge on $A$, we set for $\sigma \in G$,

$$
y\left(x_{\sigma}\right)=\frac{1}{|G|} \sum_{\rho \in G} w(f(\sigma, \rho)) .
$$

We extend $y$ to a value function on $A$ by letting

$$
y\left(\sum_{\sigma \in G} c_{\sigma} x_{\sigma}\right)=\min _{\sigma \in G}\left(w\left(c_{\sigma}\right)+y\left(x_{\sigma}\right)\right) .
$$


Proposition 4.2. The value function $y$ is a v-gauge on A, and there is a canonical isomorphism

$$
\operatorname{gr}(A) \cong_{g}\left(\operatorname{gr}(K) / \operatorname{gr}(F), G^{\prime}, f^{\prime}\right) .
$$

Proof. Let $\left(a_{i}\right)_{1 \leq i \leq n}$ be any splitting base of $w$ on $K$ as an $F$-norm. Then, it follows from the definition in (4.2) that $\left(a_{i} x_{\sigma} \mid 1 \leq i \leq n, \sigma \in G\right)$ is a splitting base for $y$, so that $y$ is an $F$-norm on $A$. Note that $w$ is invariant under the action of $G$ on $K$, since $w$ is the unique extension of $v$ to $K$. Thus, when we apply $w$ to the basic cocycle equation $f(\sigma, \tau) f(\sigma \tau, \rho)=\sigma(f(\tau, \rho)) f(\sigma, \tau \rho)$ and sum over all $\rho \in G$, we obtain in $\Gamma$,

$$
|G| w(f(\sigma, \tau))+|G| y\left(x_{\sigma \tau}\right)=|G| y\left(x_{\tau}\right)+|G| y\left(x_{\sigma}\right) .
$$

Since $\Gamma$ is torsion-free this yields

$$
y\left(x_{\sigma}\right)+y\left(x_{\tau}\right)=w(f(\sigma, \tau))+y\left(x_{\sigma \tau}\right),
$$

for all $\sigma, \tau \in G$. Therefore, for any $i, j, \sigma, \tau$ we have

$$
\begin{aligned}
y\left(\left(a_{i} x_{\sigma}\right)\left(a_{j} x_{\tau}\right)\right) & =y\left(a_{i} \sigma\left(a_{j}\right) f(\sigma, \tau) x_{\sigma \tau}\right)=w\left(a_{i}\right)+w\left(a_{j}\right)+w(f(\sigma, \tau))+y\left(x_{\sigma \tau}\right) \\
& =w\left(a_{i}\right)+w\left(a_{j}\right)+y\left(x_{\sigma}\right)+y\left(x_{\tau}\right)=y\left(a_{i} x_{\sigma}\right)+y\left(a_{j} x_{\tau}\right) .
\end{aligned}
$$

By Lemma 1.2 it follows that $y$ is surmultiplicative.

Now consider $\operatorname{gr}(A)$. Since $\left(a_{i}^{\prime}\right)_{1 \leq i \leq n}$ is a homogeneous $\operatorname{gr}(F)$-base for $\operatorname{gr}(K)$ and $\left(\left(a_{i} x_{\sigma}\right)^{\prime} \mid 1 \leq i \leq n, \sigma \in G\right)$ is a homogeneous $\operatorname{gr}(F)$-base of $\operatorname{gr}(A)$, and since $\left(a_{i} x_{\sigma}\right)^{\prime}=a_{i}^{\prime} x_{\sigma}^{\prime}$ by (4.2) , we have $\operatorname{gr}(A)=\bigoplus_{\sigma \in G} \operatorname{gr}(K) x_{\sigma}^{\prime}$. For any $c \in K^{\times}$and $\sigma \in G$, we have $y\left(x_{\sigma} c\right)=y\left(\sigma(c) x_{\sigma}\right)=w(c)+y\left(x_{\sigma}\right)$; hence, in $\operatorname{gr}(A)$,

$$
x_{\sigma}^{\prime} c^{\prime}=\left(x_{\sigma} c\right)^{\prime}=\left(\sigma(c) x_{\sigma}\right)^{\prime}=\sigma(c)^{\prime} x_{\sigma}^{\prime}=\sigma^{\prime}\left(c^{\prime}\right) x_{\sigma}^{\prime} .
$$

Moreover, formula (4.3) shows that $x_{\sigma}^{\prime} x_{\tau}^{\prime}=f(\sigma, \tau)^{\prime} x_{\sigma \tau}^{\prime}$ for all $\sigma, \tau \in G$. Therefore, $\operatorname{gr}(A) \cong_{g}\left(\operatorname{gr}(K) / \operatorname{gr}(F), G^{\prime}, f^{\prime}\right)$. It follows that $\operatorname{gr}(A)$ is a graded central simple $\operatorname{gr}(F)$-algebra; hence $y$ is a tame $F$-gauge on $A$.

We can describe $A_{0}$ to some extent for this $A$. The value function $y$ yields a map

$$
\lambda: G \rightarrow \Gamma / \Gamma_{K} \quad \text { given by } \quad \sigma \mapsto y\left(x_{\sigma}\right)+\Gamma_{K},
$$

and (4.3) shows that $\lambda$ is a group homomorphism. Let $H=\operatorname{ker}(\lambda)$. Write $A=$ $\bigoplus_{\sigma \in G} K z_{\sigma}$, where

(4.5) $z_{\sigma}=d_{\sigma} x_{\sigma}$, with the $d_{\sigma} \in K^{\times}$chosen so that $w\left(d_{\sigma}\right)=-y\left(x_{\sigma}\right)$ if $\sigma \in H$.

Thus, $d_{\sigma} d_{\tau}=g(\sigma, \tau) d_{\sigma \tau}$, where $g(\sigma, \tau)=d_{\sigma} \sigma\left(d_{\tau}\right) d_{\sigma \tau}^{-1} f(\sigma, \tau)$, so $g$ is a 2-cocycle cohomologous to $f$. We have $y\left(z_{\rho}\right)=0$ for $\rho \in H$, and the analogue to (4.3) for $g$ shows that $w(g(\rho, \tau))=0$ for all $\rho, \tau \in H$. For $\sigma \notin H$, because $y\left(z_{\sigma}\right) \notin \Gamma_{K}$ the summand $\operatorname{gr}(K) z_{\sigma}^{\prime}$ makes no contribution to $A_{0}$. Thus,

$$
A_{0}=\bigoplus_{\rho \in H} K_{0} z_{\rho}^{\prime} .
$$

This $A_{0}$ is semisimple, as $\operatorname{gr}(A)$ is simple, see (2.3), but it need not be a crossed product algebra, nor even simple, depending on how $H$ acts on $K_{0}$. Recall that $K_{0}=\bar{K}$. Because the extension of $v$ to $K$ is indecomposed, each $\sigma \in G$ induces an automorphism $\widetilde{\sigma}$ of $\bar{K}$ which coincides with the restriction of $\sigma^{\prime}$ to $K_{0}$. 
Proposition 4.3. In the situation just described, where $A=(K / F, G, f)=$ $\bigoplus K x_{\sigma}$ and $H=\operatorname{ker}(\lambda)$ for $\lambda$ as in (4.4), suppose that each $\rho \in H$ induces a dif$\sigma \in G$

ferent automorphism of $\bar{K}$. Then, $Z\left(A_{0}\right)=K_{0}^{H}$, the subfield of $K_{0}$ fixed by $H$, and $A_{0}=\bigoplus_{\rho \in H} K_{0} z_{\rho}^{\prime}$ is a crossed product algebra over $Z\left(A_{0}\right)$. Let $E$ be the graded division algebra Brauer-equivalent to $\operatorname{gr}(A)$. Then, $\Gamma_{E}=\Gamma_{\operatorname{gr}(A)}=\Gamma_{K}+\left\langle y\left(x_{\sigma}\right) \mid \sigma \in G\right\rangle$ and $E_{0}$ is the division algebra Brauer-equivalent to $A_{0}$. The map $\theta_{\operatorname{gr}(A)}: \Gamma_{E} / \Gamma_{F} \rightarrow$ $\mathcal{G}\left(Z\left(A_{0}\right) / F_{0}\right)$ induces $\tilde{\theta}: \Gamma_{\operatorname{gr}(A)} / \Gamma_{K} \rightarrow \mathcal{G}\left(K_{0}^{H} / F_{0}\right)$, and we have a commutative diagram

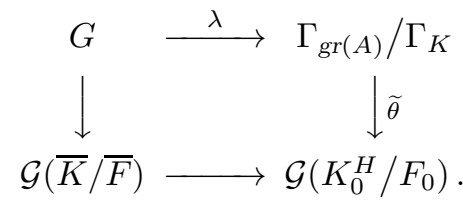

Proof. Since each $\rho \in H$ induces a different automorphism of $K_{0}=\bar{K}$, it is clear that $A_{0}=\bigoplus_{\rho \in H} K_{0} z_{\rho}^{\prime}$ is a crossed product algebra over its center $K_{0}^{H}=\bar{K}^{H}$. Clearly, $\Gamma_{\operatorname{gr}(A)}=\Gamma_{K}+\left\langle y\left(x_{\sigma}\right) \mid \sigma \in G\right\rangle$. Since $A_{0}$ is simple, we have $\Gamma_{E}=\Gamma_{\operatorname{gr}(A)}$ and $\theta_{E}=\theta_{\operatorname{gr}(A)}$, by Cor. 2.3. Furthermore, since $A_{0}$ has only one simple component, for any $\gamma \in \Gamma_{E}, \theta_{E}\left(\gamma+\Gamma_{F}\right)$ is the automorphism of $Z\left(A_{0}\right)$ induced by conjugation by any $a \in A_{\gamma} \cap A^{\times}$. If $\gamma \in \Gamma_{K}$, then $a$ can be chosen in $\operatorname{gr}(K)$, and the conjugation is trivial, as $Z\left(A_{0}\right) \subseteq K_{0}$. Hence, $\theta_{\operatorname{gr}(A)}$ induces $\widetilde{\theta}$. For each $\gamma \in \Gamma_{\operatorname{gr}(A)}$, there is $\sigma \in G$ with $\lambda\left(z_{\sigma}\right) \equiv \gamma\left(\bmod \Gamma_{K}\right)$. Then $\widetilde{\theta}\left(\gamma+\Gamma_{K}\right)$ is given by conjugation by $z_{\sigma}^{\prime}$ on $K_{0}^{H}$, which coincides with $\left.\widetilde{\sigma}\right|_{K}{ }^{H}$. Therefore, diagram (4.6) is commutative, where the left map is $\sigma \mapsto \widetilde{\sigma}$ and the bottom map is restriction of the automorphism from $\bar{K}=K_{0}$ to $\bar{K}^{H}=K_{0}^{H}$.

Now consider the unramified case of the preceding discussion. Suppose the field $K$ is Galois over $F$, and suppose $F$ has a valuation $v$ which has a unique and unramified (and defectless) extension to a valuation $w$ of $K$. So, $\bar{K}$ is Galois over $\bar{F}$ and $\mathcal{G}(\bar{K} / \bar{F}) \cong \mathcal{G}(K / F)$. Let $G=\mathcal{G}(K / F)$. The short exact sequence of trivial $G$-modules

$$
0 \rightarrow \Gamma_{F} \rightarrow \Gamma \rightarrow \Gamma / \Gamma_{F} \rightarrow 0
$$

yields a connecting homomorphism $\partial: H^{1}\left(G, \Gamma / \Gamma_{F}\right) \rightarrow H^{2}\left(G, \Gamma_{F}\right)$. In fact, $\partial$ is an isomorphism, since for the divisible torsion-free group $\Gamma$ we have $H^{1}(G, \Gamma)=$ $H^{2}(G, \Gamma)=\{0\}$. Thus, we have a succession of maps

$$
H^{2}\left(G, K^{\times}\right) \rightarrow H^{2}\left(G, \Gamma_{F}\right) \stackrel{\partial^{-1}}{\longrightarrow} H^{1}\left(G, \Gamma / \Gamma_{F}\right)=\operatorname{Hom}\left(G, \Gamma / \Gamma_{F}\right),
$$

where the left map is induced by the $G$-module homomorphism $w: K^{\times} \rightarrow \Gamma_{K}=\Gamma_{F}$.

Corollary 4.4. Let $K$ be an unramified and defectless Galois extension field of $F$ with respect to the valuation $v$ on $F$. Let $G=\mathcal{G}(K / F)$, and take any $f \in$ $Z^{2}\left(G, K^{\times}\right)$. Let $A=(K / F, G, f)=\bigoplus_{\sigma \in G} K x_{\sigma}$, and let $D$ be the division algebra Brauer-equivalent to $A$. Let $y$ be the tame F-gauge on $A$ defined in (4.2) above, and let $\lambda$ be as defined in (4.4), let $H=\operatorname{ker}(\lambda)$, and the $z_{\sigma}$ as defined in (4.5). So, 
for the graded simple ring $\operatorname{gr}_{y}(A)$, we have $A_{0}=\bigoplus_{\rho \in H} K_{0} z_{\rho}^{\prime}$, as above, and $A_{0}$ is a crossed product algebra over $\bar{K}^{H}$. Furthermore,

(i) The map $\lambda$ of (4.4) is the image of $[f] \in H^{2}\left(G, K^{\times}\right)$under the maps of (4.7).

(ii) $v$ extends to a valuation on $D$ iff $m s\left(A_{0}\right)=m s(A)$.

(iii) Suppose $v$ extends to $D$. Then $\Gamma_{D} / \Gamma_{F}=i m(\lambda), Z(\bar{D})=\bar{K}^{H}, \bar{D}$ is the division algebra Brauer-equivalent to $A_{0}$, and $\theta_{D}: \Gamma_{D} / \Gamma_{F} \rightarrow \mathcal{G}(Z(\bar{D}) / \bar{F})$ is the isomorphism which is the inverse to the composite map $\mathcal{G}(Z(\bar{D}) / \bar{F}) \cong$ $G / H \stackrel{\cong}{\rightarrow} \Gamma_{D} / \Gamma_{F}$ induced by $\lambda$.

Proof. Note that Prop. 4.3 applies here, since the map $\mathcal{G}(K / F) \rightarrow \mathcal{G}(\bar{K} / \bar{F})$ is injective. Hence, $A_{0}$ is a crossed product with center $\bar{K}^{H}$.

(i) follows from (4.3) above and the definition of the connecting homomorphism in group cohomology.

For (ii), we have $m s(\operatorname{gr}(A))=m s\left(A_{0}\right)$, as $A_{0}$ is simple, by Cor. 2.3. But, by Theorem 4.1, $v$ extends to $D$ iff $m s(A)=m s(\operatorname{gr}(A))$. This proves (ii).

(iii) Suppose $v$ extends to $D$. Let $E=\operatorname{gr}(D)$. By Theorem 4.1, we have $\operatorname{gr}(A) \cong_{g}$ $\operatorname{End}_{E}(N)$ for some graded right $E$-vector space $N$. Then, using Cor. 2.3 and Prop. 4.3, we have $\Gamma_{D} / \Gamma_{F}=\Gamma_{E} / \Gamma_{F}=\Gamma_{g r(A)} / \Gamma_{F}=i m(\lambda)$. Also, $\bar{D}=E_{0}$, which is the division algebra Brauer-equivalent to $A_{0}$, so $Z(\bar{D}) \cong Z\left(A_{0}\right)=K_{0}^{H}=\bar{K}^{H}$. Finally, again using Cor. 2.3, we have $\theta_{D}=\theta_{E}=\theta_{\operatorname{gr}(A)}$. In the commutative diagram (4.6), $\widetilde{\theta}=\theta_{\operatorname{gr}(A)}$, as $\Gamma_{K}=\Gamma_{F}$. The diagram shows that the surjective map $\widetilde{\theta}$ is also injective, as $\operatorname{ker}(\lambda)=H=\operatorname{ker}(\widetilde{\theta} \circ \lambda)$. So, $\widetilde{\theta}$ is an isomorphism, and the diagram shows that $\widetilde{\theta}$ is the inverse of the isomorphism induced by $\lambda$.

In the context of Cor. 4.4, if the valuation $v$ on $F$ is Henselian, then $v$ always extends to $D$, so Cor. 4.4 applies. It yields a new proof of [JW, Th. 5.6(b)] for inertially split division algebras over Henselian fields, which is significantly simpler and more direct than previous proofs. It does not use generalized crossed products, as in the proof in $\mathrm{JW}$, nor Dubrovin valuation rings, as in the proof in $\mathrm{MW}$, Cor. 3.7].

4.2. Tensor products of symbol algebras. Let $A$ be a finite-dimensional algebra over an arbitrary field $F$. Recall from [TW, §2] (see also [T] that an armature of $A$ is an abelian subgroup $\mathcal{A} \subset A^{\times} / F^{\times}$such that $|\mathcal{A}|=[A: F]$ and $\left\{a \in A \mid a F^{\times} \in \mathcal{A}\right\}$ spans $A$ as an $F$-vector space.

For example, suppose $F$ contains a primitive $n$-th root of unity $\omega$ for some $n \geq 2$, and $A$ is a symbol algebra of degree $n$, i.e. an $F$-algebra generated by two elements $i, j$ subject to the relations $i^{n} \in F^{\times}, j^{n} \in F^{\times}$, and $i j=\omega j i$. The images in $A^{\times} / F^{\times}$of the standard generators $i, j$ generate an armature of $A$. More generally, in a tensor product of symbol algebras the images of the products of standard generators generate an armature. Tensor products of symbol algebras can actually be characterized by the existence of armatures of a certain type; see [TW, Prop. 2.7].

Although tensor products of symbol algebras are the main case of interest to us, we first consider commutative algebras. Let $\mathcal{Z}$ be an armature of a commutative $F$-algebra $Z$. Suppose $F$ contains a primitive $s$-th root of unity for some multiple $s$ of the exponent $\exp (\mathcal{Z})$, and let $\mu_{s} \subseteq F$ denote the group of $s$-th roots of unity. 
Since $\operatorname{char}(F) \nmid s$, we have $|\mathcal{Z}| \neq 0$ in $F$. Let $\pi: Z^{\times} \rightarrow Z^{\times} / F^{\times}$be the canonical map and let

$$
X=\pi^{-1}(\mathcal{Z}) \subseteq Z^{\times} .
$$

Since $\exp (\mathcal{Z})$ divides $s$, we have $x^{s} \in F^{\times}$for all $x \in X$. Therefore, there is a commutative diagram with exact rows:

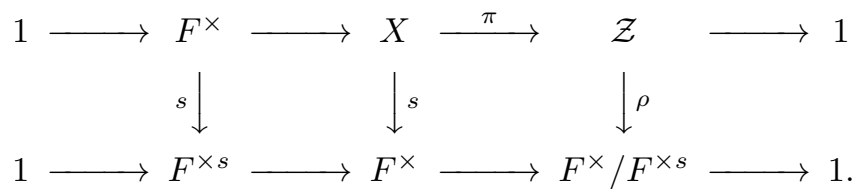

Let $\mathcal{K}=\operatorname{ker}(\rho)$ and $\mathcal{L}=\operatorname{im}(\rho)$, and let $L=F\left(\left\{\sqrt[s]{c} \mid c F^{\times s} \in \mathcal{L}\right\}\right)$ be the $s$-Kummer extension field of $F$ associated with $\mathcal{L}$. Also let $G=\operatorname{Hom}\left(\mathcal{Z}, \mu_{s}\right)$, the character group of $\mathcal{Z}$, and let $H \subseteq G$ be the subgroup orthogonal to $\mathcal{K}$,

$$
H=\{\chi \in G \mid \chi(k)=1 \text { for all } k \in \mathcal{K}\} .
$$

Also let $r=|\mathcal{K}|=|G: H|$, and let $K \subseteq Z$ be the subalgebra spanned by $\pi^{-1}(\mathcal{K})$. The following proposition extends [TW, Lemma 2.9]:

Proposition 4.5. The F-algebra $Z$ is G-Galois, and contains $r$ primitive idempotents $e_{1}, \ldots, e_{r}$, which form an $F$-base of $K$ and are conjugate under the $G$-action. The isotropy subgroup of any $e_{i}$ is $H$, and $e_{i} Z \cong L$ is a Galois extension of $F$ with Galois group isomorphic to $H$. In particular, $Z \cong L^{r}$, a direct product of $r$ copies of $L$.

Proof. For each $z \in \mathcal{Z}$, choose $x_{z} \in X$ such that $\pi\left(x_{z}\right)=z$. By definition of an armature, $\left(x_{z}\right)_{z \in \mathcal{Z}}$ is an $F$-base of $Z$. Let ${ }_{s} X=\left\{x \in X \mid x^{s}=1\right\}$. Applying the snake lemma to (4.8), we get the exact sequence

$$
1 \rightarrow \mu_{s} \rightarrow{ }_{s} X \stackrel{\pi}{\rightarrow} \mathcal{K} \rightarrow 1
$$

Since $\mu_{s}$ is a cyclic group of order $s$ in the finite abelian group ${ }_{s} X$ of exponent $s$, this exact sequence splits. Therefore, we may assume that the elements $x_{z}$ satisfy

$$
x_{k} x_{k^{\prime}}=x_{k k^{\prime}} \quad \text { for } k, k^{\prime} \in \mathcal{K} \text {. }
$$

In particular, $x_{1}=1$. In the base $\left(x_{z}\right)_{z \in \mathcal{Z}}$, the matrix of multiplication by $x_{z}$ is monomial, and the corresponding permutation is multiplication by $z$ in $\mathcal{Z}$. This permutation has no fixed point if $z \neq 1$; hence the trace map $T_{Z / F}: Z \rightarrow F$ satisfies

$$
T_{Z / F}\left(x_{1}\right)=|\mathcal{Z}| \neq 0 \quad \text { and } \quad T_{Z / F}\left(x_{z}\right)=0 \quad \text { for } z \neq 1 .
$$

It is then straightforward to check that the bilinear trace form on $Z$ is not degenerate; hence $Z$ is étale. An action of $G$ on $Z$ is defined by

$$
\chi * x_{z}=\chi(z) x_{z} \quad \text { for } z \in \mathcal{Z} .
$$

If $z \neq 1$, there exists $\chi \in G$ with $\chi(z) \neq 1$; hence $F \subseteq Z$ is the set of fixed points under the $G$-action. Since $|G|=|\mathcal{Z}|=[Z: F]$, it follows that $Z$ is a $G$-Galois $F$-algebra; see [KMRT, Sec. 18B].

Now, consider $e=\frac{1}{r} \sum_{k \in \mathcal{K}} x_{k}$. In view of (4.9), we have $e x_{k}=e$ for all $k \in \mathcal{K}$; hence $e^{2}=e$. Let $z_{1}, \ldots, z_{m} \in \mathcal{Z}$ be representatives of the cosets modulo $\mathcal{K}$. Since $x_{z_{i}} x_{k} \in x_{z_{i} k} F^{\times}$, the products $x_{z_{i}} x_{k}$ for $k \in \mathcal{K}$ and $i=1, \ldots, m$ form a base of 
$Z$. For $i=1, \ldots, m$ the product $e x_{z_{i}}$ is in the $F$-span of $\left(x_{z_{i}} x_{k}\right)_{k \in \mathcal{K}}$; hence $e x_{z_{1}}$, $\ldots, e x_{z_{m}}$ are linearly independent. These elements span $e Z$ since $e x_{z_{i}} x_{k}=e x_{z_{i}}$ for $k \in \mathcal{K}$; hence they form a base of $e Z$. Let

$$
e X=\{e x \mid x \in X\}=\bigcup_{i=1}^{m} e x_{z_{i}} F^{\times} \subseteq(e Z)^{\times} .
$$

Mapping $e x$ to $\rho \pi(x) \in \mathcal{L}$ defines a surjective map $e X \rightarrow \mathcal{L}$ with kernel $e F^{\times}$; hence $\mathcal{L}$ may be identified with an armature of $e Z$. By [TW, Lemma 2.9], it follows that $e Z \cong L$. Since $L$ is a field, $e$ is a primitive idempotent in $Z$. From the definition of $e$, it is clear that $H \subseteq G$ is the subgroup of elements that leave $e$ fixed; hence the orbit of $e$ has $r$ elements, which span $K$. The structure theorem of Galois algebras (see [KMRT, (18.18)]) shows that the primitive idempotents of $Z$ are the conjugates of $e$, and that $e Z$ is $H$-Galois. The proof is thus complete.

Remark 4.6. The $G$-structure of $Z$ can be made explicit by [KMRT, Prop. (18.18)]: it is an induced algebra $Z=\operatorname{Ind}_{H}^{G}(e Z)$.

For an armature $\mathcal{A}$ of an arbitrary finite-dimensional $F$-algebra $A$, there is an associated armature pairing

$$
\beta_{\mathcal{A}}: \mathcal{A} \times \mathcal{A} \rightarrow \mu(F) \quad \text { given by }\left(a F^{\times}, b F^{\times}\right) \mapsto a b a^{-1} b^{-1},
$$

where $\mu(F)$ denotes the group of roots of unity in $F$. It is shown in [TW], $\S 2$ ] that $\beta_{\mathcal{A}}$ is a well-defined symplectic bimultiplicative pairing, and if $\beta_{\mathcal{A}}$ is nondegenerate, then $A$ is isomorphic to a tensor product of symbol algebras. Conversely, in any tensor product of symbol algebras the standard generators generate an armature whose associated pairing is nondegenerate. For any subgroup $\mathcal{B} \subseteq \mathcal{A}$, we let

$$
\mathcal{B}^{\perp}=\left\{a \in \mathcal{A} \mid \beta_{\mathcal{A}}(a, b)=1 \text { for all } b \in \mathcal{B}\right\},
$$

which is a subgroup of $\mathcal{A}$. Note that when $\beta_{\mathcal{A}}$ is nondegenerate, i.e., $\mathcal{A} \cap \mathcal{A}^{\perp}=\{1\}$, we have $|\mathcal{B}|\left|\mathcal{B}^{\perp}\right|=|\mathcal{A}|$.

We now fix the setting we will consider for the rest of the paper. Let $A$ be an $F$-algebra with an armature $\mathcal{A}$ such that $\beta_{\mathcal{A}}$ is nondegenerate. Let $s=\exp (\mathcal{A})$. The nondegeneracy of $\beta_{\mathcal{A}}$ implies that $\mu_{s} \subseteq F$. We denote by $\pi: A^{\times} \rightarrow A^{\times} / F^{\times}$the canonical map. Let $v: F \rightarrow \Gamma \cup\{\infty\}$ be a valuation on $F$. Assume that $\operatorname{char}(\bar{F}) \nmid s$. Hence, the group $\mu_{s}=\mu_{s}(F)$ of $s$-th roots of unity in $F$ maps bijectively to $\mu_{s}(\bar{F})$. We build a tame $F$-gauge on $A$ using the armature $\mathcal{A}$. For this, define functions

$$
w: \pi^{-1}(\mathcal{A}) \rightarrow \Gamma \text { given by } w(x)=\frac{1}{s} v\left(x^{s}\right)
$$

and

$$
\widetilde{w}: \mathcal{A} \rightarrow \Gamma / \Gamma_{F}, \text { the map induced by } w .
$$

Note that $w$ and $\widetilde{w}$ are group homomorphisms, since the commutators of elements of $\pi^{-1}(\mathcal{A})$ are roots of unity, hence elements in $F^{\times}$of value 0 . Clearly, $\left.w\right|_{F^{\times}}=v$. For each $a \in \mathcal{A}$, pick $x_{a} \in A^{\times}$such that $\pi\left(x_{a}\right)=a$. Then $\left(x_{a}\right)_{a \in \mathcal{A}}$ is an $F$-base of $A$. Define an $F$-norm $y: A \rightarrow \Gamma \cup\{\infty\}$ by

$$
y\left(\sum_{i=1}^{n} \lambda_{a} x_{a}\right)=\min _{a \in \mathcal{A}}\left(v\left(\lambda_{a}\right)+w\left(x_{a}\right)\right) .
$$

The definition of $y$ depends on $\mathcal{A}$, but is independent of the choice of the $x_{a}$. 
Proposition 4.7. The F-norm $y$ is a tame F-gauge on $A$ and $\Gamma_{A}$ is determined by

$$
\Gamma_{A} / \Gamma_{F}=\widetilde{w}(\mathcal{A}) .
$$

The graded algebra $\operatorname{gr}(A)$ has an armature isometric to $\left(\mathcal{A}_{1} \beta_{\mathcal{A}}\right)$. Moreover, every homogeneous component of $\operatorname{gr}(A)$ contains an invertible element; hence the subgroup $\Delta_{\operatorname{gr}(A)} \subseteq \Gamma_{\operatorname{gr}(A)}$ defined in Sec. 2 coincides with $\Gamma_{A}$, and the map $\theta_{\operatorname{gr}(A)}$ of (2.5) is a homomorphism

$$
\theta_{\operatorname{gr}(A)}: \Gamma_{A} / \Gamma_{F} \rightarrow \operatorname{Aut}\left(Z\left(A_{0}\right)\right) .
$$

Proof. Note that $\left.y\right|_{\pi^{-1}(\mathcal{A})}=w$. Hence, for all $a, b \in \mathcal{A}$, we have $y\left(x_{a} x_{b}\right)=$ $y\left(x_{a}\right)+y\left(x_{b}\right) ;$ so for the image $x_{a}^{\prime}$ of $x_{a}$ in $\operatorname{gr}(A),\left(x_{a} x_{b}\right)^{\prime}=x_{a}^{\prime} x_{b}^{\prime}$. It follows by Lemma 1.2 that $y$ is surmultiplicative, and that $\pi^{-1}(\mathcal{A})$ maps to a subgroup of $\operatorname{gr}(A)^{\times}$. Furthermore, $\left(x_{a}^{\prime}\right)_{a \in \mathcal{A}}$ is a $\operatorname{gr}(F)$-base of $\operatorname{gr}(A)$ by Prop. 1.1(i), since $\left(x_{a}\right)_{a \in \mathcal{A}}$ is an $F$-splitting base of $A$. Thus, the image $\mathcal{A}^{\prime}$ of $\left\{x_{a}^{\prime} \mid a \in \mathcal{A}\right\}$ in $\operatorname{gr}(A)^{\times} / \operatorname{gr}(F)^{\times}$could be called a graded armature for $\operatorname{gr}(A)$. The map $\mathcal{A} \rightarrow \mathcal{A}^{\prime}$ given by $x_{a} F^{\times} \mapsto x_{a}^{\prime} \operatorname{gr}(F)^{\times}$is clearly a group isomorphism and also an isometry between the armature pairings $\beta_{\mathcal{A}}$ and $\beta_{\mathcal{A}^{\prime}}$ when we identify $\mu_{s}(F)$ with $\mu_{s}(\bar{F})$. The pairing $\beta_{\mathcal{A}^{\prime}}$ is therefore nondegenerate, so an argument analogous to the ungraded one in [TW, Prop. 2.7] shows that $\operatorname{gr}(A)$ is isomorphic to a graded tensor product of graded symbol algebras over $\operatorname{gr}(F)$. Since it is easy to see that graded symbol algebras are graded central simple $\operatorname{gr}(F)$-algebras (by a slight variation of the ungraded argument), it follows that $\operatorname{gr}(A)$ is graded central simple over $\operatorname{gr}(F)$. Thus, $y$ is a tame $F$-gauge on $A$.

Our next goal is to describe the degree 0 component $A_{0} \subseteq \operatorname{gr}(A)$, which is a semisimple algebra over $F_{0}=\bar{F}$. For this, we consider

$$
\mathcal{B}=\operatorname{ker}(\widetilde{w}) \subseteq \mathcal{A}, \quad \mathcal{Z}=\mathcal{B} \cap \mathcal{B}^{\perp},
$$

and denote by $Z \subseteq A$ the subalgebra spanned by $\pi^{-1}(\mathcal{Z})$. Since $\beta_{\mathcal{A}}$ is trivial on $\mathcal{Z}$, the $F$-algebra $Z$ is commutative.

Proposition 4.8. The $F_{0}$-algebra $A_{0}$ has an armature $\mathcal{B}_{0}$ canonically isomorphic to $\mathcal{B}$ with armature pairing $\beta_{\mathcal{B}_{0}}$ isometric to the restriction of $\beta_{\mathcal{A}}$ to $\mathcal{B}$. Its center $Z\left(A_{0}\right)$ is the degree 0 component of $Z$, i.e. $Z\left(A_{0}\right)=Z_{0}$; it is an $\left(\mathcal{A} / \mathcal{Z}^{\perp}\right)$-Galois $F_{0}$-algebra. For the map $\psi: \mathcal{A} / \mathcal{Z}^{\perp} \hookrightarrow A_{F}\left(Z\left(A_{0}\right)\right)$ given by the Galois action, the following diagram is commutative:

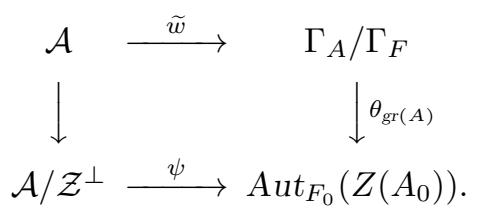

Proof. We first fix a convenient choice of $x_{a} \in A^{\times}$such that $\pi\left(x_{a}\right)=a \in \mathcal{A}$ : for $b \in \mathcal{B}$, we choose $x_{b}$ such that $w\left(x_{b}\right)=0$. As observed in the proof of Prop. 4.7. $\left(x_{a}^{\prime}\right)_{a \in \mathcal{A}}$ is a homogeneous $\operatorname{gr}(F)$-base of $\operatorname{gr}(A)$. We have $y\left(x_{a}\right)=0$ if and only if $a \in \mathcal{B}$; hence $\left(x_{b}^{\prime}\right)_{b \in \mathcal{B}}$ is an $F_{0}$-base of $A_{0}$. We have

$$
x_{b}^{\prime} x_{c}^{\prime} x_{b}^{\prime-1} x_{c}^{\prime-1}=\overline{\beta_{\mathcal{A}}(b, c)} \in \mu_{s}\left(F_{0}\right) ;
$$

hence $\mathcal{B}_{0}=\left\{x_{b}^{\prime} F_{0}^{\times} \mid b \in \mathcal{B}\right\}$ is an armature of the $F_{0}$-algebra $A_{0}$, with armature pairing isometric to the restriction of $\beta_{\mathcal{A}}$ to $\mathcal{B}$. It follows that $Z\left(A_{0}\right)$ is spanned 
by $\left(x_{z}^{\prime}\right)_{z \in \mathcal{Z}}$; hence $Z\left(A_{0}\right)=Z_{0}$. As in Prop. 4.5, $Z_{0}$ is $\operatorname{Hom}\left(\mathcal{Z}, \mu_{s}\right)$-Galois, for the action defined by

$$
\chi * x_{z}^{\prime}=\chi(z) x_{z}^{\prime} \quad \text { for } \chi \in \operatorname{Hom}\left(\mathcal{Z}, \mu_{s}\right) \text { and } z \in \mathcal{Z} .
$$

Since $\beta_{\mathcal{A}}$ is nondegenerate, the map $\mathcal{A} \rightarrow \operatorname{Hom}\left(\mathcal{Z}, \mu_{s}\right)$ that carries $a \in \mathcal{A}$ to the character $\chi$ defined by

$$
\chi(z)=\beta_{\mathcal{A}}(a, z) \quad \text { for } z \in \mathcal{Z}
$$

is surjective, and its kernel is $\mathcal{Z}^{\perp}$. Therefore, $\mathcal{A} / \mathcal{Z}^{\perp} \cong \operatorname{Hom}\left(\mathcal{Z}, \mu_{s}\right)$, and $Z$ is $\left(\mathcal{A} / \mathcal{Z}^{\perp}\right)$-Galois. For $z \in \mathcal{Z}$ and $a \in \mathcal{A}$, (4.11) yields

$$
\chi * x_{z}=\beta_{\mathcal{A}}(a, z) x_{z}=x_{a} x_{z} x_{a}^{-1} .
$$

Hence the action of $\chi$ on $Z$ is conjugation by $x_{a}$; the induced action on $Z_{0}$ is conjugation by $a^{\prime}$, so diagram (4.10) commutes.

The arguments above also show that $\left\{x_{z}^{\prime} F_{0}^{\times} \mid z \in \mathcal{Z}\right\} \subseteq Z_{0}^{\times} / F_{0}^{\times}$is an armature of $Z_{0}$ which is isomorphic to $\mathcal{Z}$. We may use this armature to determine the primitive idempotents of $Z_{0}$ as in Prop. 4.5. consider the map

$$
\rho_{0}: \mathcal{Z} \rightarrow F_{0}^{\times} / F_{0}^{\times s} \quad \text { given by } z \mapsto x_{z}^{\prime s} F_{0}^{\times s} .
$$

Let $\mathcal{K}_{0}=\operatorname{ker}\left(\rho_{0}\right), \mathcal{L}_{0}=\operatorname{im}\left(\rho_{0}\right)$, and $r_{0}=\left|\mathcal{K}_{0}\right|$. Also let $E$ be the graded division $\operatorname{gr}(F)$-algebra Brauer-equivalent to $\operatorname{gr}(A)$.

Proposition 4.9. The $F_{0}$-algebra $Z\left(A_{0}\right)$ contains $r_{0}$ primitive idempotents, which are conjugate in $\operatorname{gr}(A)$. Letting $t$ denote the index of any simple component of $A_{0}$, we have

$$
\operatorname{ms}(\operatorname{gr}(A))=r_{0} t^{-1} \sqrt{|\mathcal{B}: \mathcal{Z}|} .
$$

Moreover, $Z\left(E_{0}\right)$ is the s-Kummer extension of $F_{0}$ associated with $\mathcal{L}_{0}$, and $\Gamma_{E} / \Gamma_{F}$ $=\widetilde{w}\left(\mathcal{K}_{0}^{\perp}\right)$.

Proof. Prop. 4.5 shows that $Z_{0}$ contains $r_{0}$ primitive idempotents, which are conjugate under the $\operatorname{Hom}\left(\mathcal{Z}, \mu_{s}\right)$-Galois action, and whose isotropy subgroup is the orthogonal of $\mathcal{K}_{0}$ in $\operatorname{Hom}\left(\mathcal{Z}, \mu_{s}\right)$. On the other hand, Prop. 4.8 shows that the $\operatorname{Hom}\left(\mathcal{Z}, \mu_{s}\right)$-Galois action is also realized by inner automorphisms of $\operatorname{gr}(A)$ and yields an isomorphism $\operatorname{Hom}\left(\mathcal{Z}, \mu_{s}\right) \cong \mathcal{A} / \mathcal{Z}^{\perp}$ (see 44.11)) carrying the orthogonal of $\mathcal{K}_{0}$ in the character group to $\mathcal{K}_{0}^{\perp} / \mathcal{Z}^{\perp}$. Therefore, the primitive idempotents of $Z_{0}$ are conjugate in $\operatorname{gr}(A)$. Propositions 2.2 and 4.8 show that the inverse image of $\Gamma_{E} / \Gamma_{F}$ in $\mathcal{A}$ is $\mathcal{K}_{0}^{\perp}$; hence, $\Gamma_{E} / \Gamma_{F}=\widetilde{w}\left(\mathcal{K}_{0}^{\perp}\right)$.

The center $Z\left(E_{0}\right)$ is isomorphic to the simple components of $Z_{0}=Z\left(A_{0}\right)$ (see Prop. 2.2), and hence also to the $s$-Kummer extension of $F_{0}$ associated with $\mathcal{L}_{0}$, by Prop. 4.5.

Finally, we compute the matrix size of $\operatorname{gr}(A)$. First, note that $\widetilde{w}: \mathcal{K}_{0}^{\perp} \rightarrow \Gamma_{E} / \Gamma_{F}$ is surjective with kernel $\mathcal{B}$; hence $\left|\Gamma_{E}: \Gamma_{F}\right|=\left|\mathcal{K}_{0}^{\perp}\right||\mathcal{B}|^{-1}$. Since the pairing $\beta_{\mathcal{A}}$ is nondegenerate, we have $\left|\mathcal{K}_{0}^{\perp}\right|\left|\mathcal{K}_{0}\right|=|\mathcal{A}|$; hence

$$
\left|\Gamma_{E}: \Gamma_{F}\right|=\frac{|\mathcal{A}|}{r_{0}|\mathcal{B}|}=\frac{[\operatorname{gr}(A): \operatorname{gr}(F)]}{r_{0}|\mathcal{B}|} .
$$

On the other hand,

$$
\left[E_{0}: F_{0}\right]=t^{2}\left[Z\left(E_{0}\right): F_{0}\right]=t^{2}\left|\mathcal{L}_{0}\right|=t^{2}|\mathcal{Z}| r_{0}^{-1} .
$$


Since $[E: \operatorname{gr}(F)]=\left[E_{0}: F_{0}\right]\left|\Gamma_{E}: \Gamma_{F}\right|$, it follows that

$$
[E: \operatorname{gr}(F)]=\frac{t^{2}|\mathcal{Z}|[\operatorname{gr}(A): \operatorname{gr}(F)]}{r_{0}^{2}|\mathcal{B}|}=\frac{t^{2}[\operatorname{gr}(A): \operatorname{gr}(F)]}{r_{0}^{2}|\mathcal{B}: \mathcal{Z}|} .
$$

Since $\operatorname{ms}(\operatorname{gr}(A))=\sqrt{[\operatorname{gr}(A): \operatorname{gr}(F)][E: \operatorname{gr}(F)]^{-1}}$, formula (4.12) follows.

Let $D$ be the division $F$-algebra Brauer-equivalent to $A$. By combining Theorem 4.1 and Prop. 4.9, we readily obtain a criterion for the extension of the valuation $v$ on $F$ to $D$ :

Corollary 4.10. The valuation $v$ on $F$ extends to a valuation on $D$ if and only if $m s(A)=r_{0} t^{-1} \sqrt{|\mathcal{B}: \mathcal{Z}|}$. When this occurs, $\bar{D}=E_{0}$ and $\Gamma_{D}=\Gamma_{E}$.

Note that when it exists the valuation on $D$ is necessarily tame since $\operatorname{char}(\bar{F}) \nmid$ $s=\exp (\mathcal{A})$ while $\operatorname{deg}(D) \mid \operatorname{deg}(A)=\sqrt{|\mathcal{A}|}$.

Finally, we consider the case where $v$ extends to a valuation $v_{D}$ on $D$ that is totally ramified over $F$. Recall from [TW, Sec. 3] that in this case there is a canonical pairing $C_{D}: \Gamma_{D} / \Gamma_{F} \times \Gamma_{D} / \Gamma_{F} \rightarrow \mu(\bar{F})$ defined by $C_{D}\left(\gamma_{1}+\Gamma_{F}, \gamma_{2}+\Gamma_{F}\right)=$ $\overline{x_{1} x_{2} x_{1}^{-1} x_{2}^{-1}}$ for any $x_{1}, x_{2} \in D^{\times}$with $v_{D}\left(x_{i}\right)=\gamma_{i}$ for $i=1,2$.

Proposition 4.11. The valuation $v$ on $F$ extends to a valuation on $D$ that is tamely and totally ramified over $F$ if and only if $\operatorname{deg}(D)=\sqrt{\left|\mathcal{B}^{\perp}: \mathcal{Z}\right|}$. When this occurs, we have $\Gamma_{D} / \Gamma_{F}=\widetilde{w}\left(\mathcal{B}^{\perp}\right)$ and $\widetilde{w}$ defines an isometry from $\mathcal{B}^{\perp} / \mathcal{Z}$ with the nondegenerate pairing induced by $\beta_{\mathcal{A}}$ onto $\Gamma_{D} / \Gamma_{F}$ with the pairing $C_{D}$.

Proof. Since $[\operatorname{gr}(A): \operatorname{gr}(F)]=|\mathcal{A}|=|\mathcal{B}|\left|\mathcal{B}^{\perp}\right|$, equation (4.13) yields

$$
[E: \operatorname{gr}(F)]=t^{2}\left|\mathcal{B}^{\perp}\right||\mathcal{Z}| r_{0}^{-2}=t^{2}\left|\mathcal{B}^{\perp}: \mathcal{Z}\right||\mathcal{Z}|^{2} r_{0}^{-2}=t^{2}\left|\mathcal{L}_{0}\right|^{2}\left|\mathcal{B}^{\perp}: \mathcal{Z}\right| \text {. }
$$

On the other hand, Theorem 4.1 yields $[D: F] \geq[E: \operatorname{gr}(F)]$. Therefore, if $[D: F]=$ $\left|\mathcal{B}^{\perp}: \mathcal{Z}\right|$, then we must have $[D: F]=[E: \operatorname{gr}(F)]$ and $t=\left|\mathcal{L}_{0}\right|=1$; hence $v$ extends to a valuation on $D$ that is totally ramified over $F$.

For the converse, we apply Cor. 4.10 with $t=1$ and $r_{0}=|\mathcal{Z}|$ and obtain $\operatorname{ms}(A)=\sqrt{|\mathcal{B}||\mathcal{Z}|}$. Since $[A: F]=[D: F] m s(A)^{2}$ and $[A: F]=|\mathcal{A}|=|\mathcal{B}|\left|\mathcal{B}^{\perp}\right|$, it follows that $[D: F]=\left|\mathcal{B}^{\perp}: \mathcal{Z}\right|$.

For the rest of the proof, assume $v$ extends to a valuation on $D$ that is tamely and totally ramified over $F$. Then $\operatorname{gr}(D) \cong_{g} E$, and $r_{0}=|\mathcal{Z}|$; hence $\mathcal{K}_{0}=\mathcal{Z}$ and $\Gamma_{D} / \Gamma_{F}=\Gamma_{E} / \Gamma_{F}=\widetilde{w}\left(\mathcal{Z}^{\perp}\right)$ by Prop. 4.9. Since $\mathcal{Z}=\mathcal{B} \cap \mathcal{B}^{\perp}$, we have $\mathcal{Z}^{\perp}=\mathcal{B}+\mathcal{B}^{\perp}$; hence $\widetilde{w}\left(\mathcal{Z}^{\perp}\right)=\widetilde{w}\left(\mathcal{B}^{\perp}\right)$ since $\mathcal{B}=\operatorname{ker}(\widetilde{w})$. We may identify the canonical pairing $C_{D}$ with the pairing $C$ on $\Gamma_{E} / \Gamma_{F}$ given by

$$
C\left(\gamma+\Gamma_{F}, \delta+\Gamma_{F}\right)=\xi \eta \xi^{-1} \eta^{-1} \quad \text { for any nonzero } \xi \in E_{\gamma}, \eta \in E_{\delta} .
$$

In order to relate $C$ to $\beta_{\mathcal{A}}$, we identify a copy of $E$ in $\operatorname{gr}(A)$. First, we choose for each $a \in \mathcal{A}$ an element $x_{a} \in A^{\times}$such that $\pi\left(x_{a}\right)=a$. As in the proof of Prop. 4.8, we choose $x_{b}$ such that $w\left(x_{b}\right)=0$ for $b \in \mathcal{B}$. Note that $\mathcal{Z}_{0}=\left\{x_{z}^{\prime} F_{0}^{\times} \mid z \in \mathcal{Z}\right\}$ is an armature of $Z_{0}$ which is isomorphic to $\mathcal{Z}$. Since $\mathcal{Z}=\operatorname{ker}\left(\rho_{0}\right), \mathcal{Z}_{0}$ is the kernel of the $s$-power map $\mathcal{Z}_{0} \rightarrow F_{0}^{\times} / F_{0}^{\times s}$. Therefore, the proof of Prop. 4.5 shows that after scaling $x_{z}$ for $z \in \mathcal{Z}$ by suitable units in $F^{\times}$we may assume $x_{z_{1}}^{\prime} x_{z_{2}}^{\prime}=x_{z_{1} z_{2}}^{\prime}$ for $z_{1}, z_{2} \in \mathcal{Z}$.

As in the proof of Prop. 4.5, we consider $e=\frac{1}{|\mathcal{Z}|} \sum_{z \in \mathcal{Z}} x_{z}^{\prime}$, which is a primitive idempotent in $Z_{0}$ such that $e x_{z}^{\prime}=e$ for $z \in \mathcal{Z}$. For $a \in \mathcal{A}$, we have

$$
x_{a}^{\prime} e x_{a}^{\prime-1}=\frac{1}{|\mathcal{Z}|} \sum_{z \in \mathcal{Z}} \overline{\beta_{\mathcal{A}}(a, z)} x_{z}^{\prime},
$$


which is $e$ if $a \in \mathcal{Z}^{\perp}$, and is another primitive idempotent of $Z_{0}$ if $a \notin \mathcal{Z}^{\perp}$. Thus, $e x_{a}^{\prime} e=e x_{a}^{\prime}=x_{a}^{\prime} e$ if $a \in \mathcal{Z}^{\perp}$, and $e\left(x_{a}^{\prime} e x_{a}^{\prime-1}\right)=0$, hence $e x_{a}^{\prime} e=0$, if $a \notin \mathcal{Z}^{\perp}$. Therefore, $\operatorname{egr}(A) e$ is spanned by $\left(e x_{a}^{\prime}\right)_{a \in \mathcal{Z}^{\perp}}$. If $a_{1}, \ldots, a_{r} \in \mathcal{Z}^{\perp}$ are in different cosets modulo $\mathcal{Z}$, then $e x_{a_{1}}^{\prime}, \ldots, e x_{a_{r}}^{\prime}$ are linearly independent since each $e x_{a_{i}}^{\prime}$ lies in the span of $\left(x_{z a_{i}}^{\prime}\right)_{z \in \mathcal{Z}}$. Let $n=|\mathcal{B}: \mathcal{Z}|, m=\left|\mathcal{B}^{\perp}: \mathcal{Z}\right|$, and let $b_{1}, \ldots, b_{n} \in \mathcal{B}$ (resp. $c_{1}, \ldots, c_{m} \in \mathcal{B}^{\perp}$ ) be representatives of the various cosets of $\mathcal{B}$ (resp. $\mathcal{B}^{\perp}$ ) modulo $\mathcal{Z}$. Since $\mathcal{Z}^{\perp}=\mathcal{B}+\mathcal{B}^{\perp}$ and $\mathcal{Z}=\mathcal{B} \cap \mathcal{B}^{\perp}$, we have $\mathcal{Z}^{\perp} / \mathcal{Z}=(\mathcal{B} / \mathcal{Z}) \oplus\left(\mathcal{B}^{\perp} / \mathcal{Z}\right)$; hence $\left\{b_{i} c_{j} \mid 1 \leq i \leq n, 1 \leq j \leq m\right\}$ is a set of representatives of the various cosets of $\mathcal{Z}^{\perp}$ modulo $\mathcal{Z}$. For $i=1, \ldots, n$ and $j=1, \ldots, m$, let

$$
\xi_{i}=e x_{b_{i}}^{\prime}=x_{b_{i}}^{\prime} e \in e A_{0} \subseteq \operatorname{egr}(A) e \quad \text { and } \quad \eta_{j}=e x_{c_{j}}^{\prime}=x_{c_{j}}^{\prime} e \in \operatorname{egr}(A) e .
$$

Then $\left(\xi_{i} \eta_{j} \mid 1 \leq i \leq n, 1 \leq j \leq m\right)$ is a $\operatorname{gr}(F)$-base of $\operatorname{egr}(A) e$. Moreover, $\xi_{i} \eta_{j}=\eta_{j} \xi_{i}$ since $\beta_{\mathcal{A}}\left(b_{i}, c_{j}\right)=1$. Therefore, the graded subalgebras $B, B^{\prime} \subseteq \operatorname{egr}(A) e$ spanned respectively by $\xi_{1}, \ldots, \xi_{n}$ and by $\eta_{1}, \ldots, \eta_{m}$ centralize each other, and

$$
\operatorname{egr}(A) e \cong \otimes_{g r(F)} B^{\prime} .
$$

The degree of each $\xi_{i}$ is 0 since $b_{i} \in \mathcal{B}=\operatorname{ker}(\widetilde{w})$; hence $B=B_{0} \otimes_{F_{0}} \operatorname{gr}(F)$. On the other hand, the degree of $\eta_{j}$ is 0 if and only if $\eta_{j} \in e F_{0}$ since $\mathcal{B}^{\perp} \cap \mathcal{B}=\mathcal{Z}$. Therefore, $e A_{0}=B_{0}$. This algebra is split by hypothesis. Therefore, $B$ is split and $\operatorname{gr}(A)$ is Brauer-equivalent to $B^{\prime}$. Since $\left[B^{\prime}: \operatorname{gr}(F)\right]=\left|\mathcal{B}^{\perp}: \mathcal{Z}\right|=[E: \operatorname{gr}(F)]$, we may identify $B^{\prime}$ with $E$. Clearly, under this identification the canonical pairing on $\Gamma_{E} / \Gamma_{F}$ coincides with the pairing on $\mathcal{B}^{\perp} / \mathcal{Z}$ induced by $\beta_{\mathcal{A}}$.

Remarks 4.12. (i) The descriptions of $\bar{D}$ and $\Gamma_{D}$ in Cor. 4.10 (with additional information from Propositions 4.8 and 4.9 ) were given in $\mathrm{W}_{3}$, Th. 1], and proved using Morandi value functions. The proof given here is easier and more direct. By Prop. 2.5 the tame gauge $y$ defined here is a Morandi value function (so the associated valuation ring $A^{\geq 0}$ is a Dubrovin valuation ring) if and only if $\left|\mathcal{B}_{0}\right|=1$, i.e., if and only if $A^{\geq 0}$ has a unique maximal two-sided ideal.

(ii) Suppose the valuation $v$ on $F$ is strictly Henselian; i.e., $v$ is Henselian and $\bar{F}$ is separably closed. Then, in the setting of Prop. 4.11 with $\operatorname{char}(\bar{F}) \nmid \exp (\mathcal{A}), v$ necessarily extends to a valuation on the division algebra $D$ Brauer-equivalent to $A$, and $D$ is totally and tamely ramified over $F$. In that situation, the description of the canonical pairing on $D$ (which then determines $D$ up to isomorphism by [TW, Prop. 4.2]) was given in [TW, Th. 4.3], with a more difficult proof.

\section{REFERENCES}

[AS] S. A. Amitsur and L. W. Small, Prime ideals in PI-rings, J. Algebra, 62 (1980), 358-383. MR.563234 (81c:16027)

$\left[\mathrm{Bl}_{1}\right] \quad$ M. Boulagouaz, The graded and tame extensions, pp. 27-40 in Commutative Ring Theory (Fès, 1992) (P. J. Cahen et al., eds.), Lecture Notes in Pure and Applied Math., No. 153, Marcel Dekker, New York, 1994. MR1261874 (94k:12010)

$\left[\mathrm{Bl}_{2}\right] \quad$ M. Boulagouaz, Le gradué d'une algèbre à division valuée, Comm. Algebra, 23 (1995), 4275-4300. MR:1351134 (96h:16047)

[B] N. Bourbaki, Elements of Mathematics, Commutative Algebra, Addison-Wesley, Reading, Mass., 1972 (English trans. of Éléments de Mathématique, Algèbre Commutative). MR0360549(50:12997)

[BT] F. Bruhat and J. Tits, Schémas en groupes et immeubles des groupes classiques sur un corps local, Bull. Soc. Math. France, 112 (1984), 259-301. MR788969 (86i:20064)

[C] P. M. Cohn, On extending valuations in division algebras, Studia Scient. Math. Hung., 16 (1981), 65-70. MR703642 (84f:16025) 
[EP] A. J. Engler and A. Prestel, Valued Fields, Springer, Berlin, 2005. MR2183496 (2007a:12005)

[G] J. Gräter, The "Defektsatz" for central simple algebras, Trans. Amer. Math. Soc., 330 (1992), 823-843. MR1034663 (92f:16018)

$\left[\mathrm{HW}_{1}\right] \quad$ Y.-S. Hwang and A. R. Wadsworth, Algebraic extensions of graded and valued fields. Comm. Algebra, 27 (1999), 821-840. MR1671995 (2000d:12006)

$\left[\mathrm{HW}_{2}\right] \quad$ Y.-S. Hwang and A. R. Wadsworth, Correspondences between valued division algebras and graded division algebras, J. Algebra, 220 (1999), 73-114. MR1713449|(2000g:16028)

[JW] B. Jacob and A. R. Wadsworth, Division algebras over Henselian fields, J. Algebra, 128 (1990), 126-179. MR 1031915(91d:12006)

[KO] M.-A. Knus and M. Ojanguren, Théorie de la Descente et Algèbres d'Azumaya, Lecture Notes in Math. 389, Springer-Verlag, Berlin-Heidelberg-New York, 1974. MR0417149 (54:5209)

[KMRT] M.-A. Knus, A.S. Merkurjev, M. Rost, and J.-P. Tignol, The Book of Involutions, Amer. Math. Soc. Coll. Pub. 44, Providence, RI, 1998. MR1632779 (2000a:16031)

[MMU] H. Marubayashi, H. Miyamoto, and A. Ueda, Non-commutative Valuation Rings and Semi-hereditary Orders, Kluwer, Dordrecht, 1997. MR 1461811 (98k:16062)

$\left[\mathrm{M}_{1}\right] \quad$ P. J. Morandi, The Henselization of a valued division algebra, J. Algebra, 122 (1989), 232-243. MR994945 (90h:12007)

$\left[\mathrm{M}_{2}\right] \quad$ P. J. Morandi, Value functions on central simple algebras, Trans. Amer. Math. Soc., 315 (1989), 605-622. MR986697 (90e:16007)

[MW] P. J. Morandi and A. R. Wadsworth, Integral Dubrovin valuation rings, Trans. Amer. Math. Soc., 315 (1989), 623-640. MR986696 (91d:16076)

[N] M. Nagata, Local rings, Interscience Publishers, a division of John Wiley \& Sons New York, 1962. MR0155856(27:5790)

[RTW] J.-F. Renard, J.-P. Tignol, and A. R. Wadsworth, Graded Hermitian forms and Springer's theorem, Indag. Math., N.S., 18 (2007), 97-134. MR2330736(2008d:11033)

[R] P. Ribenboim, Théorie des Valuations, Presses de l' Université de Montréal, Montréal, Canada, 1968. MR0249425 (40:2670)

[T] J.-P. Tignol, Sur les décompositions des algèbres à division en produit tensoriel d'algèbres cycliques, pp. 126-145 in Brauer Groups in Ring Theory and Algebraic Geometry (F. Van Oystaeyen and A. Verschoren, eds.), Lecture Notes in Math., vol. 917, SpringerVerlag, Berlin, 1982. MR657427 (83i:16020)

[TW] J.-P. Tignol and A. R. Wadsworth, Totally ramified valuations on finite-dimensional division algebras, Trans. Amer. Math. Soc., 302 (1987), 223-250. MR887507(88j:16025)

$\left[\mathrm{W}_{1}\right] \quad$ A. R. Wadsworth, Extending valuations to finite-dimensional division algebras, Proc. Amer. Math. Soc. 98 (1986), 20-22. MR848866 (87i:16025)

$\left[\mathrm{W}_{2}\right] \quad$ A. R. Wadsworth, Dubrovin valuation rings and Henselization, Math. Annalen, 283 (1989), 301-328. MR980600 (90f:16009)

$\left[\mathrm{W}_{3}\right] \quad$ A. R. Wadsworth, Valuations on tensor products of symbol algebras, pp. 275-289 in Azumaya algebras, actions, and modules, eds. D. Haile and J. Osterburg, Contemp. Math., Vol. 124, Amer. Math. Soc., Providence, RI, 1992. MR1144041|(93a:16015)

$\left[\mathrm{W}_{4}\right] \quad$ A. R. Wadsworth, Valuation theory on finite dimensional division algebras, pp. 385-449 in Valuation Theory and its Applications, Vol. I, eds. F.-V. Kuhlmann et al., Fields Inst. Commun., 32, Amer. Math. Soc., Providence, RI, 2002. MR1928379 (2003g:16023)

Institut de Mathématique Pure et Appliquée, Université catholique de Louvain, B1348 Louvain-La-Neuve, Belgium

E-mail address: jean-pierre.tignol@uclouvain.be

Department of Mathematics, University of California, San Diego, La Jolla, CaliFORNIA 92093-0112

E-mail address: arwadsworth@ucsd.edu 